\title{
Photoredox Ni-catalyzed Branch-Selective Reductive Coupling of
}

\author{
Aldehydes with 1,3-Dienes \\ Yan-Lin Li, ${ }^{\dagger,}$ Wen-Duo Li, ${ }^{\dagger, \dagger}$ Zheng-Yang Gu, ${ }^{\dagger}$ Jie Chen $^{\dagger}$ and Ji-Bao Xia ${ }^{*},{ }^{\dagger}$ \\ ${ }^{\dagger}$ State Key Laboratory for Oxo Synthesis and Selective Oxidation, Suzhou Research Institute of LICP, Lanzhou \\ Institute of Chemical Physics (LICP), University of Chinese Academy of Sciences, Chinese Academy of Sciences, \\ Lanzhou 730000, China \\ *University of Chinese Academy of Sciences, Beijing, 100049, China \\ *Corresponding Author: jibaoxia@licp.cas.cn
}

1. General Information

3. General Procedure and Characterization Data

4. Examples of Less Successful Substrates . $\mathbf{S 2 0}$

5. Control Experiments and Mechanistic Studies

6. References

7. NMR Spectra 


\section{General Information}

All of the reactions were carried out in oven-dried Schlenk tube and were manipulated in a glovebox filled with nitrogen. Thin layer chromatography (TLC) employed glass $0.25 \mathrm{~mm}$ silica gel plates. Flash chromatography columns were packed with 200-300 mesh silica gel in petroleum (bp. 30-60 ${ }^{\circ} \mathrm{C}$ ). The High Resolution MS analyses were performed on Thermo Fisher Scientific LTQ FT Ultra with DART Positive Mode or Agilent 6530 Accurate-Mass Q-TOF LC/MS with ESI mode. NMR spectra were recorded on a $400 \mathrm{MHz}$ for ${ }^{1} \mathrm{H}$ NMR and $100 \mathrm{MHz}$ for ${ }^{13} \mathrm{C} \mathrm{NMR}$, using tetramethylsilane as an internal reference and $\mathrm{CDCl}_{3}$ as solvent. Chemical shift values for protons are reported in parts per million (ppm, $\delta$ scale) downfield from tetramethylsilane and are referenced to residual proton of $\mathrm{CDCl}_{3}(\delta 7.26)$. Multiplicity is indicated by one or more of the following: $\mathrm{s}$ (singlet); $\mathrm{d}$ (doublet); $\mathrm{t}$ (triplet); $\mathrm{m}$ (multiplet); br (broad). Carbon nuclear magnetic resonance spectra $\left({ }^{13} \mathrm{C} \mathrm{NMR}\right)$ were recorded at $100 \mathrm{MHz}$. Chemical shifts for carbons are reported in parts per million (ppm, $\delta$ scale) downfield from tetramethylsilane and are referenced to the carbon resonance of $\mathrm{CDCl}_{3}(\delta$ 77.0). The photoreactors used in this research were bought from GeAoChem (Blue LEDs, light intensity $=42 \mathrm{mw} / \mathrm{cm}^{2}, 5 \mathrm{~W}$ for every light bulb; every Schlenk tube was irradiated by 1 light bulbs from the side). Aldehyde substrate 1-Tosyl-1H-pyrrole-2-carbaldehyde ${ }^{\text {la }}$, 5-(benzyloxy) pentanal $^{1 \mathrm{~b}}, 2$-phenylcyclopropane-1-carbaldehyde ${ }^{\text {lc }},(3 \mathrm{a} R, 5 R, 6 R, 6 \mathrm{a} R)-5-\left((R)-2,2-\right.$ dimethyl-1,3 $^{2}$ -dioxolan-4-yl)-2,2-dimethyltetrahydrofuro[2,3- $d][1,3]$ dioxol-6-yl 3-formylbenzoate, ${ }^{2}$ and $\operatorname{Ir}\left[\mathrm{dF}\left(\mathrm{CF}_{3}\right) \mathrm{ppy}_{2}(\mathrm{dtbbpy}) \mathrm{PF}_{6}\right.$ photocatalyst ${ }^{3}$ were prepared by known procedures. Nickel complex $\mathbf{L} 2 \mathrm{NiCl}_{2}\left(\mathbf{L} \mathbf{2}=5,5^{\prime}\right.$-dimethyl-2,2'-bipyridine) was prepared according to the reported procedure. ${ }^{4}$ All the other materials were purchased from Tokyo Chemical Industry Co., Aldrich Inc., Alfa Aesar, Adamas, or other commercial suppliers and used as received unless otherwise noted. Solvents were dried over sodium and freshly distilled prior to use. 


\section{Optimization of the Reaction Conditions}

\subsection{Table S1. The Effect of Photocatalyst ${ }^{a}$}

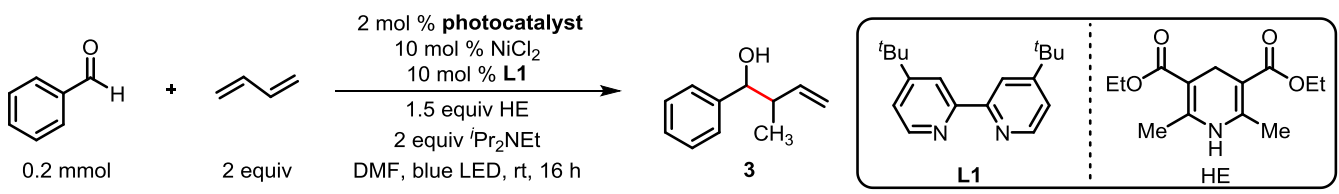

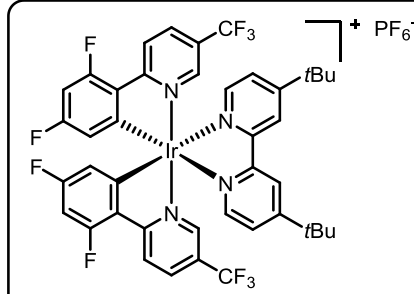

$\operatorname{Ir}\left[\mathrm{dF}\left(\mathrm{CF}_{3}\right) \mathrm{ppy}\right]_{2}(\mathrm{dtbbpy}) \mathrm{PF}_{6}(\mathrm{I})$
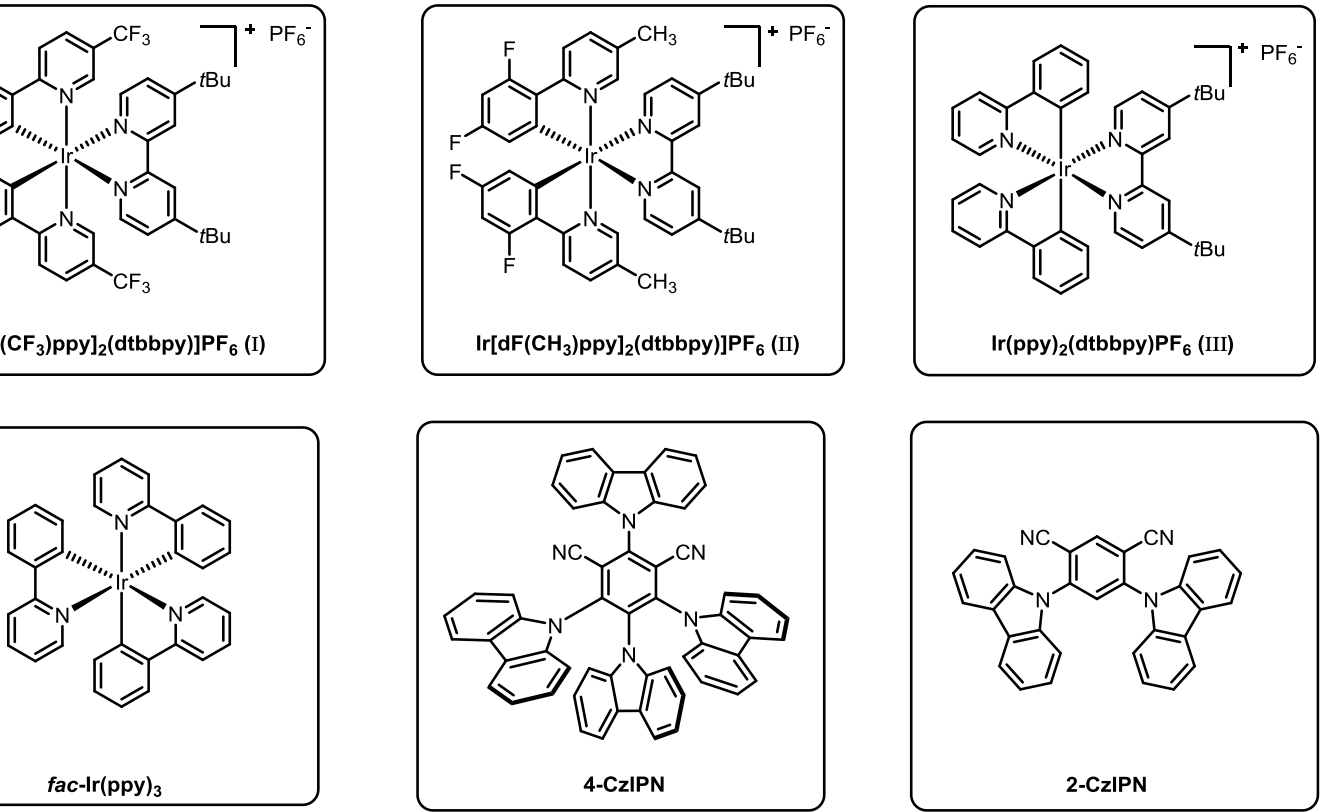

\begin{tabular}{cccc}
\hline Entry & Photocatalyst & Yield $^{b}(\%)$ & $\mathrm{dr}^{b}$ \\
\hline 1 & $\operatorname{Ir}(\text { ppy) })_{3}$ & 39 & $1.1: 1$ \\
2 & I & 50 & $1.9: 1$ \\
3 & II & 54 & $1.2: 1$ \\
4 & III & 39 & $1.4: 1$ \\
5 & 4-CzIPN & N.R. & - \\
6 & 2-CzIPN & N.R. & -
\end{tabular}

${ }^{a}$ All reaction were performed with 2 mol \% photocatalyst, $10 \mathrm{~mol} \% \mathrm{NiCl}_{2}, 10 \mathrm{~mol} \% \mathbf{L 1}, 1.5$ equiv $\mathrm{HE}, 2$ equiv ${ }^{i} \mathrm{Pr}_{2} \mathrm{NEt}$, and $5 \mathrm{~W}$ blue LED if otherwise noted. ${ }^{b}$ Determined by crude ${ }^{1} \mathrm{H}$ NMR with 1,1,2,2-tetrachloroethane as an internal standard. 


\subsection{Table S2. The Effect of Ni-catalyst ${ }^{a}$}

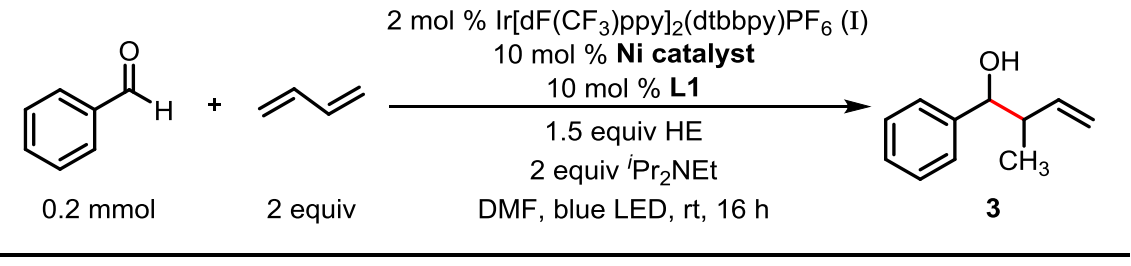

\begin{tabular}{|c|c|c|c|}
\hline & $\overbrace{\mathrm{L} 1}^{t_{\mathrm{B}}}$ & ONe & \\
\hline Entry & Ni catalyst & $\operatorname{Yield}^{b}(\%)$ & $\mathrm{dr}^{b}$ \\
\hline 1 & $\mathrm{NiCl}_{2}$ & 50 & $1.9: 1$ \\
\hline 2 & $\mathrm{NiBr}_{2}$ & 33 & $0.8: 1$ \\
\hline 3 & $\mathrm{NiCl}_{2} \cdot 6 \mathrm{H}_{2} \mathrm{O}$ & 67 & $1.7: 1$ \\
\hline 4 & $\mathrm{Ni}(\mathrm{acac})_{2}$ & 35 & $1.2: 1$ \\
\hline 5 & $\mathrm{NiF}_{2}$ & N.R. & - \\
\hline 6 & $\mathrm{Ni}\left(\mathrm{PPh}_{3}\right)_{2} \mathrm{Cl}_{2}$ & 52 & $1.2: 1$ \\
\hline 7 & $\mathrm{Ni}\left(\mathrm{PCy}_{3}\right)_{2} \mathrm{Cl}_{2}$ & trace & - \\
\hline 8 & $\mathrm{Ni}(\mathrm{dppp}) \mathrm{Cl}_{2}$ & trace & - \\
\hline
\end{tabular}

${ }^{a}$ All reaction were performed with $2 \mathrm{~mol} \% \operatorname{Ir}\left[\mathrm{dF}\left(\mathrm{CF}_{3}\right) \mathrm{ppy}\right]_{2}(\mathrm{dtbbpy}) \mathrm{PF}_{6}(\mathbf{I}), 10 \mathrm{~mol} \% \mathrm{Ni}$ catalyst, $10 \mathrm{~mol} \% \mathbf{L 1}$, 1.5 equiv $\mathrm{HE}$, and 2 equiv ${ }^{i} \mathrm{Pr}_{2} \mathrm{NEt}$, and $5 \mathrm{~W}$ blue LED if otherwise noted. ${ }^{b}$ Determined by crude ${ }^{1} \mathrm{H}$ NMR with 1,1,2,2-tetrachloroethane as an internal standard. 


\subsection{Table S3. The Effect of Ligand ${ }^{a}$}

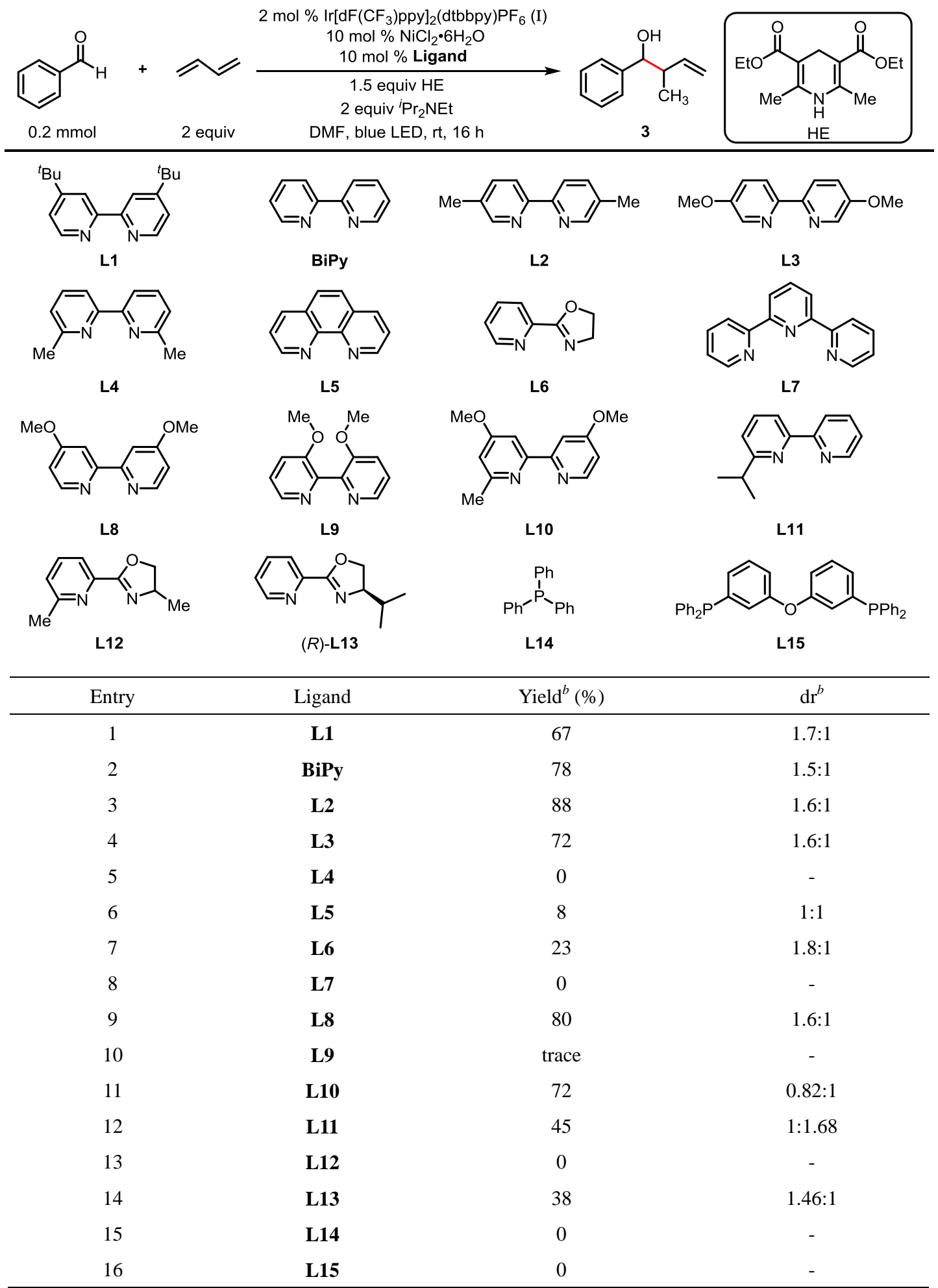

${ }^{a}$ All reaction were performed with $2 \mathrm{~mol} \% \operatorname{Ir}\left[\mathrm{dF}\left(\mathrm{CF}_{3}\right) \text { ppy }\right]_{2}(\mathrm{dtbbpy}) \mathrm{PF}_{6}(\mathbf{I}), 10 \mathrm{~mol} \% \mathrm{NiCl}_{2} \cdot 6 \mathrm{H}_{2} \mathrm{O}, 10 \mathrm{~mol} \%$ Ligand, 1.5 equiv HE, 2 equiv ${ }^{i} \operatorname{Pr}_{2} \mathrm{NEt}$, and $5 \mathrm{~W}$ blue LED if otherwise noted. ${ }^{b}$ Determined by crude ${ }^{1} \mathrm{H}$ NMR with 1,1,2,2-tetrachloroethane as an internal standard. 


\subsection{Table S4. The Effect of Hantzsch ester and Base ${ }^{a}$}

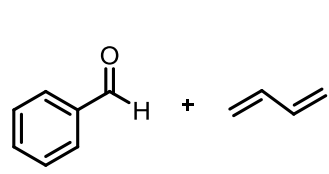

$0.2 \mathrm{mmol}$

2 equiv

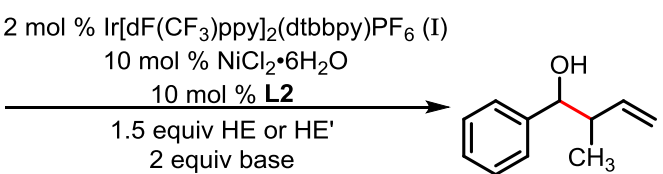

DMF, blue LED, $\mathrm{rt}, 16 \mathrm{~h}$
3

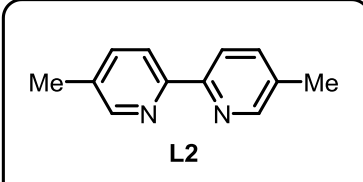

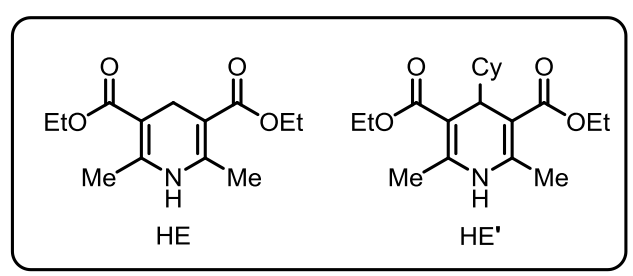

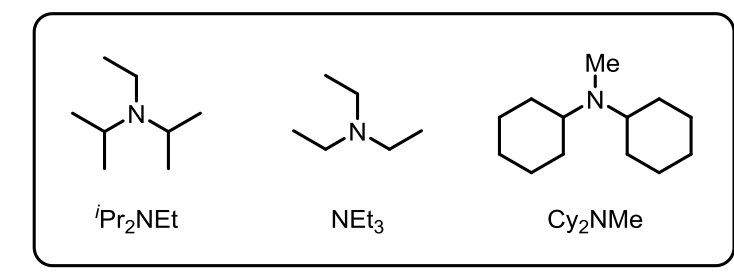

\begin{tabular}{ccccc}
\hline Entry & Hantzsch ester & Base & Yield $^{b}(\%)$ & $\mathrm{dr}^{b}$ \\
\hline 1 & $\mathrm{HE}$ & ${ }^{i} \mathrm{Pr}_{2} \mathrm{NEt}$ & 88 & $1.6: 1$ \\
2 & $\mathrm{HE}^{\prime}$ & ${ }^{i} \mathrm{Pr}_{2} \mathrm{NEt}$ & 0 & - \\
3 & $\mathrm{HE}$ & $\mathrm{NEt}_{3}$ & 50 & $1.4: 1$ \\
4 & $\mathrm{HE}$ & $\mathrm{Cy}_{2} \mathrm{NMe}$ & 67 & $1.4: 1$ \\
\hline
\end{tabular}

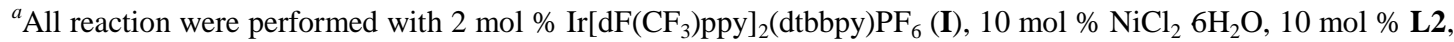
1.5 equiv Hantzsch ester, 2 equiv base, and $5 \mathrm{~W}$ blue LED if otherwise noted. ${ }^{b}$ Determined by crude ${ }^{1} \mathrm{H}$ NMR with 1,1,2,2-tetrachloroethane as an internal standard. 


\subsection{Table S5. The Effect of Solvent ${ }^{a}$}

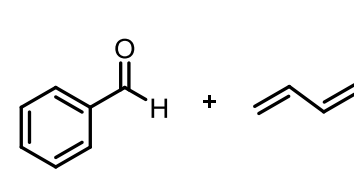

$0.2 \mathrm{mmol}$

2 equiv

$2 \mathrm{~mol} \% \operatorname{Ir}\left[\mathrm{dF}\left(\mathrm{CF}_{3}\right) \mathrm{ppy}\right]_{2}(\mathrm{dtbbpy}) \mathrm{PF}_{6}(\mathrm{I})$
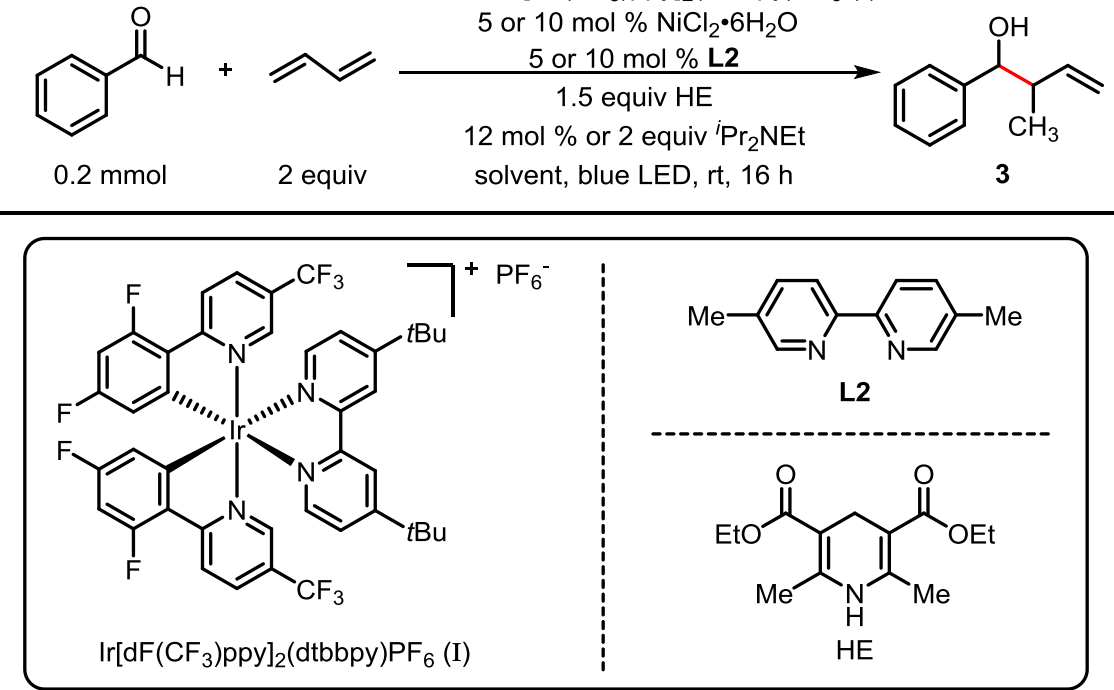

\begin{tabular}{cccc}
\hline Entry & Solvent & Yield $^{b}(\%)$ & $\mathrm{dr}^{b}$ \\
\hline 1 & DMF & 88 & $1.6: 1$ \\
2 & DMSO & 71 & $1.5: 1$ \\
3 & MeOH & 58 & $2.1: 1$ \\
4 & THF & 85 & $1.9: 1$ \\
5 & Toluene & 0 & - \\
6 & Et ${ }_{2} \mathrm{O}$ & 0 & - \\
$7^{c}$ & THF & 81 & $1.6: 1$ \\
$\mathbf{8}^{c, d}$ & THF & $\mathbf{8 6}$ & $\mathbf{1 . 9 : 1}$
\end{tabular}

${ }^{a}$ All reaction were performed with $2 \mathrm{~mol} \% \operatorname{Ir}\left[\mathrm{dF}\left(\mathrm{CF}_{3}\right) \mathrm{ppy}\right]_{2}(\mathrm{dtbbpy}) \mathrm{PF}_{6}(\mathbf{I}), 10 \mathrm{~mol} \% \mathrm{NiCl}_{2} \cdot 6 \mathrm{H}_{2} \mathrm{O}, 10 \mathrm{~mol} \% \mathbf{L 2}$, 1.5 equiv HE, 2 equiv ${ }^{i} \operatorname{Pr}_{2} \mathrm{NEt}$, and $5 \mathrm{~W}$ blue LED if otherwise noted. ${ }^{b}$ Determined by crude ${ }^{1} \mathrm{H}$ NMR with 1,1,2,2-tetrachloroethane as an internal standard. ${ }^{c}$ With $5 \mathrm{~mol} \% \mathrm{NiCl}_{2} \cdot 6 \mathrm{H}_{2} \mathrm{O}$ and 5 mol \% $\mathbf{L 2}$. ${ }^{d} \mathrm{With} 12 \mathrm{~mol} \%$ ${ }^{i} \operatorname{Pr}_{2} \mathrm{NEt}$. 


\section{General Procedure and Characterization Data}

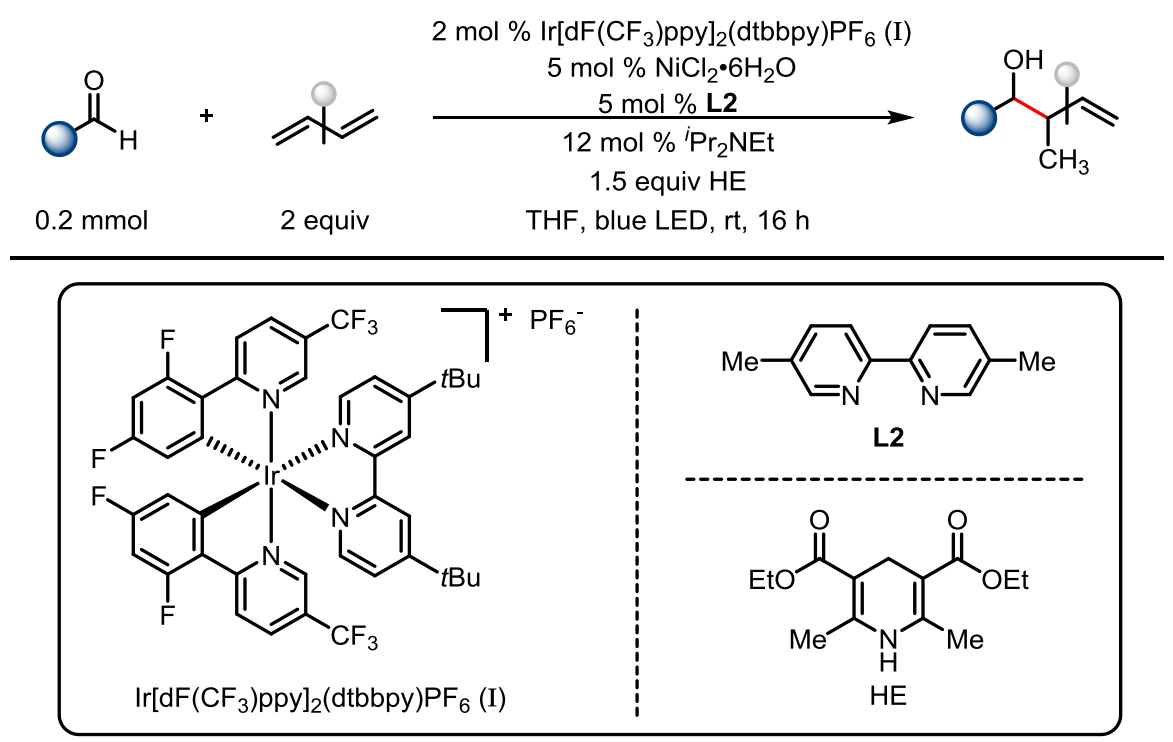

General Procedure for Photoredox Ni-catalyzed Branch-Selective Reductive Coupling of Aldehydes with 1,3-Dienes: In an argon-filled glovebox, a $25 \mathrm{~mL}$ Schlenk tube was charged with aldehyde (0.2 mmol), 1,3-diene (0.4 mmol), Hantzsch ester (HE) $(0.3$

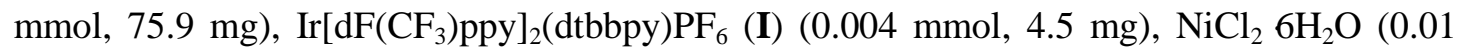
mmol, $2.4 \mathrm{mg})$, 5,5'-dimethyl-2,2'-bipyridine $\mathbf{L} 2(0.01 \mathrm{mmol}, 2.0 \mathrm{mg}),{ }^{i} \operatorname{Pr}_{2} \mathrm{NEt}(0.024 \mathrm{mmol}$, $3.1 \mathrm{mg})$ and dry THF $(2 \mathrm{~mL})$. The tube was removed from the glove box, and irradiated with blue LED $(5 \mathrm{~W})$ at room temperature for $16 \mathrm{~h}$. The mixture was then concentrated in vacuo. And the crude product was purified by flash column chromatography (silica gel, PE/EA or PE/DCM) to afford the desired product.<smiles>C=CC(C)C(O)c1ccccc1</smiles>

2-Methyl-1-phenylbut-3-en-1-ol (3). ${ }^{5}$ Synthesized according to the general procedure with benzaldehyde $(0.2 \mathrm{mmol}, 21.2 \mathrm{mg}$ ) and 1,3-butadiene ( $0.4 \mathrm{mmol}, 0.2 \mathrm{ml}, 2 \mathrm{M}$ in THF solution) irradiated with blue LED for $16 \mathrm{~h}$. Purified by flash column chromatography on silica gel (PE : $\mathrm{DCM}=3: 1)$ to give 3 as a colorless oil ( $26.9 \mathrm{mg}, 83 \%$ yield). syn isomer: anti isomer $=66$ : 34. ${ }^{1} \mathrm{H}$ NMR (400 MHz, $\left.\mathrm{CDCl}_{3}\right)$ syn isomer: $\delta 7.32-7.23(\mathrm{~m}, 5 \mathrm{H}), 5.85-5.7(\mathrm{~m}, 1 \mathrm{H}), 5.20-$ $5.02(\mathrm{~m}, 2 \mathrm{H}), 4.59(\mathrm{~d}, J=5.2 \mathrm{~Hz}, 1 \mathrm{H}), 2.61-2.53(\mathrm{~m}, 1 \mathrm{H}), 2.02$ (brs, $1 \mathrm{H}), 1.00$ (d, $J=6.8 \mathrm{~Hz}$ $3 \mathrm{H})$; anti isomers: $4.35(\mathrm{~d}, J=8.0 \mathrm{~Hz}, 1 \mathrm{H}), 2.5-2.43(\mathrm{~m}, 1 \mathrm{H}), 2.20(\mathrm{brs}, 1 \mathrm{H}), 0.86(\mathrm{~d}, J=6.8$ $\mathrm{Hz}, 3 \mathrm{H}) .{ }^{13} \mathrm{C} \mathrm{NMR}\left(100 \mathrm{MHz}, \mathrm{CDCl}_{3}\right)$ syn isomer: $\delta 142.6,140.3,128.0,127.3,126.5,115.4$, 77.3, 44.6, 14.0; anti isomers: $\delta 142.5,140.6,128.2,127.6,126.8,116.7,77.9,46.2,16.5$.<smiles>C=CC(C)C(O)c1ccc(C)cc1</smiles> 
2-Methyl-1-(p-tolyl)but-3-en-1-ol (4). ${ }^{6}$ Synthesized according to the general procedure with 4-methylbenzaldehyde $(0.2 \mathrm{mmol}, 24.0 \mathrm{mg})$ and 1,3-butadiene $(0.4 \mathrm{mmol}, 0.2 \mathrm{ml}, 2 \mathrm{M}$ in THF solution) irradiated with blue LED for $16 \mathrm{~h}$. Purified by flash column chromatography on silica gel (PE : DCM = 3: 1) to give 4 as a colorless oil (32.0 mg, 91\% yield). syn isomer : anti isomer $=67: 33 .{ }^{1} \mathrm{H}$ NMR $\left(400 \mathrm{MHz}, \mathrm{CDCl}_{3}\right)$ syn isomer: $\delta 7.23-7.11(\mathrm{~m}, 4 \mathrm{H}), 5.85-$ $5.69(\mathrm{~m}, 1 \mathrm{H}), 5.20-5.01(\mathrm{~m}, 2 \mathrm{H}), 4.53(\mathrm{~d}, J=4.4 \mathrm{~Hz}, 1 \mathrm{H}), 2.59-2.51(\mathrm{~m}, 1 \mathrm{H}), 2.33(\mathrm{~s}, 3 \mathrm{H})$, 2.01 (brs, $1 \mathrm{H}), 1.00(\mathrm{~d}, J=6.8 \mathrm{~Hz}, 3 \mathrm{H})$; anti isomer: $4.30(\mathrm{~d}, J=8.0 \mathrm{~Hz}, 1 \mathrm{H}), 2.48-2.41(\mathrm{~m}$, $1 \mathrm{H}), 2.17$ (brs, $1 \mathrm{H}), 0.85(\mathrm{~d}, J=6.8 \mathrm{~Hz}, 3 \mathrm{H}) .{ }^{13} \mathrm{C} \mathrm{NMR}\left(100 \mathrm{MHz}, \mathrm{CDCl}_{3}\right)$ syn isomer: $\delta$ $140.4,139.6,136.9,128.7,126.4,115.3,77.2,44.6,21.0,14.2$; anti isomer: $\delta 140.8,139.5$, $137.2,128.9,126.7,116.5,77.7,46.1,21.0,16.5$.

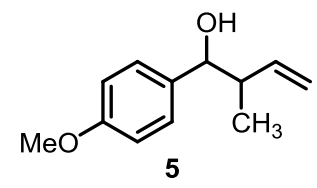

1-(4-Methoxyphenyl)-2-methylbut-3-en-1-ol (5). ${ }^{7}$ Synthesized according to the general procedure with 4-methoxybenzaldehyde $(0.2 \mathrm{mmol}, 27.2 \mathrm{mg})$ and 1,3-butadiene $(0.4 \mathrm{mmol}$, $0.2 \mathrm{ml}, 2 \mathrm{M}$ in THF solution) irradiated with blue LED for $16 \mathrm{~h}$. Purified by flash column chromatography on silica gel (PE : DCM $=2: 1$ ) to give 5 as a colorless oil ( $35.3 \mathrm{mg}, 92 \%$ yield). syn isomer : anti isomer $=71: 29 .{ }^{1} \mathrm{H}$ NMR $\left(400 \mathrm{MHz}, \mathrm{CDCl}_{3}\right)$ syn isomer: $\delta 7.25-$ $7.19(\mathrm{~m}, 2 \mathrm{H}), 6.89-6.84(\mathrm{~m}, 2 \mathrm{H}), 5.85-5.67(\mathrm{~m}, 1 \mathrm{H}), 5.21-4.99(\mathrm{~m}, 2 \mathrm{H}), 4.52(\mathrm{~d}, J=5.6$ $\mathrm{Hz}, 1 \mathrm{H}), 3.79$ (s, 3H), $2.58-2.50(\mathrm{~m}, 1 \mathrm{H}), 2.02$ (brs, 1H), $1.01(\mathrm{~d}, J=6.8 \mathrm{~Hz}, 3 \mathrm{H})$; anti isomer: $4.29(\mathrm{~d}, J=8.0 \mathrm{~Hz}, 1 \mathrm{H}), 2.47-2.40(\mathrm{~m}, 1 \mathrm{H}), 2.18(\mathrm{brs}, 1 \mathrm{H}), 0.84(\mathrm{~d}, J=6.8 \mathrm{~Hz}, 3 \mathrm{H})$. ${ }^{13} \mathrm{C}$ NMR (100 MHz, $\mathrm{CDCl}_{3}$ ) syn isomer: $\delta 158.9,140.4,134.8,127.7,115.3,113.6,77.1$, 55.2, 44.6, 14.4; anti isomer: $\delta 159.1,140.9,134.6,127.9,116.5,113.4,77.5,55.2,46.3$, 16.5 .

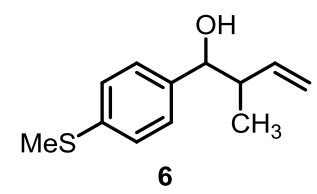

2-Methyl-1-(4-(methylthio)phenyl)but-3-en-1-ol (6). Synthesized according to the general procedure with 4 -(methylthio)benzaldehyde $(0.2 \mathrm{mmol}, 30.4 \mathrm{mg})$ and 1,3-butadiene $(0.4$ mmol, $0.2 \mathrm{ml}, 2 \mathrm{M}$ in THF solution) irradiated with blue LED for $16 \mathrm{~h}$. Purified by flash column chromatography on silica gel (PE : EA $=10: 1)$ to give 6 as a pale yellow oil (22.4 $\mathrm{mg}, 54 \%$ yield). The relative configuration of 6 is determined by comparing the $J$ value of the two doublet peaks (4.56 ppm, $4.32 \mathrm{ppm}$ ) of benzylic C-H in the ${ }^{1} \mathrm{H}$ NMR. The $J$ value of the anti isomer $(8.0 \mathrm{~Hz})$ is greater than the syn isomer $(5.6 \mathrm{~Hz})$. syn isomer : anti isomer $=69: 31$. ${ }^{1} \mathrm{H}$ NMR (400 MHz, $\mathrm{CDCl}_{3}$ ) syn isomer: $\delta 7.26-7.22(\mathrm{~m}, 4 \mathrm{H}), 5.84-5.69(\mathrm{~m}, 1 \mathrm{H}), 5.22-$ $5.02(\mathrm{~m}, 2 \mathrm{H}), 4.56(\mathrm{~d}, J=5.6 \mathrm{~Hz}, 1 \mathrm{H}), 2.60-2.51(\mathrm{~m}, 1 \mathrm{H}), 2.47$ (s, 3H), 1.96 (brs, $1 \mathrm{H}), 1.00$ $(\mathrm{d}, J=6.8 \mathrm{~Hz}, 3 \mathrm{H})$; anti isomer: $4.32(\mathrm{~d}, J=8.0 \mathrm{~Hz}, 1 \mathrm{H}), 2.46-2.35(\mathrm{~m}, 1 \mathrm{H}), 2.15$ (brs, $1 \mathrm{H})$, $0.87(\mathrm{~d}, J=6.8 \mathrm{~Hz}, 3 \mathrm{H}) .{ }^{13} \mathrm{C}$ NMR $\left(100 \mathrm{MHz}, \mathrm{CDCl}_{3}\right)$ syn isomer: $\delta 140.1,139.6,137.3$, 127.0, 126.4, 115.6, 76.9, 44.6, 15.9, 14.0; anti isomer: $\delta 140.5,139.4,137.6,127.3,126.6$, 
116.8, 77.5, 46.2, 15.9, 16.5. HRMS (ESI) Calcd for $\mathrm{C}_{12} \mathrm{H}_{16} \mathrm{OSNa}[\mathrm{M}+\mathrm{Na}]^{+}$231.0820, Found 231.0818 .

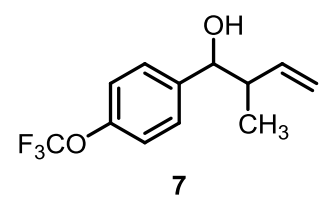

2-Methyl-1-(4-(trifluoromethoxy)phenyl)but-3-en-1-ol (7). Synthesized according to the general procedure with 4-(trifluoromethoxy)benzaldehyde $(0.2 \mathrm{mmol}, 38.0 \mathrm{mg})$ and 1,3-butadiene ( $0.4 \mathrm{mmol}, 0.2 \mathrm{ml}, 2 \mathrm{M}$ in THF solution) irradiated with blue LED for $16 \mathrm{~h}$. Purified by flash column chromatography on silica gel (PE : DCM $=3: 1$ ) to give 7 as a pale yellow oil (45.2 $\mathrm{mg}, 92 \%$ yield). The relative configuration of $\mathbf{7}$ is determined according to the same method as 6. syn isomer : anti isomer $=63: 37 .{ }^{1} \mathrm{H} \mathrm{NMR}\left(400 \mathrm{MHz}, \mathrm{CDCl}_{3}\right)$ syn isomer: $\delta 7.35-7.16(\mathrm{~m}, 4 \mathrm{H}), 5.81-5.69(\mathrm{~m}, 1 \mathrm{H}), 5.21-5.03(\mathrm{~m}, 2 \mathrm{H}), 4.61(\mathrm{~d}, J=1.6 \mathrm{~Hz}$, $1 \mathrm{H}), 2.58-2.50(\mathrm{~m}, 1 \mathrm{H}), 2.12$ (brs, $1 \mathrm{H}), 0.98(\mathrm{~d}, J=6.8 \mathrm{~Hz}, 3 \mathrm{H})$; anti isomer: 4.36 (d, $J=$ $8.0 \mathrm{~Hz}, 1 \mathrm{H}), 2.48-2.39(\mathrm{~m}, 1 \mathrm{H}), 2.30$ (brs, $1 \mathrm{H}), 0.87(\mathrm{~d}, J=6.8 \mathrm{~Hz}, 3 \mathrm{H}) .{ }^{13} \mathrm{C}$ NMR $(100$ $\left.\mathrm{MHz}, \mathrm{CDCl}_{3}\right)$ syn isomer: $\delta 148.4,141.2,139.9,127.8,120.5(\mathrm{q}, J=256 \mathrm{~Hz}), 120.5,115.9$, 76.5, 44.6, 13.8; anti isomer: $\delta 148.6,141.1,140.1,128.2,120.5$ (q, $J=256 \mathrm{~Hz}), 120.7,117.2$, 77.1, 46.4, 16.4. ${ }^{19} \mathrm{~F}$ NMR $\left(377 \mathrm{MHz}, \mathrm{CDCl}_{3}\right) \delta-57.92$, -57.93. HRMS (ESI) Calcd for $\mathrm{C}_{12} \mathrm{H}_{13} \mathrm{~F}_{3} \mathrm{O}_{2} \mathrm{Na}[\mathrm{M}+\mathrm{Na}]^{+}$269.0765, Found 269.0759.

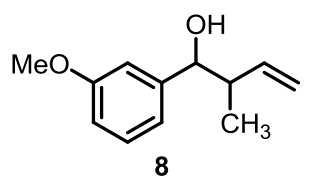

1-(3-Methoxyphenyl)-2-methylbut-3-en-1-ol (8). ${ }^{7}$ Synthesized according to the general procedure with 3-methoxybenzaldehyde $(0.2 \mathrm{mmol}, 27.2 \mathrm{mg})$ and 1,3-butadiene $(0.4 \mathrm{mmol}$, $0.2 \mathrm{ml}, 2 \mathrm{M}$ in THF solution) irradiated with blue LED for $16 \mathrm{~h}$. Purified by flash column chromatography on silica gel (PE : DCM $=3: 1)$ to give 8 as a pale yellow oil $(35.3 \mathrm{mg}, 92 \%$ yield). syn isomer : anti isomer $=63: 37 .{ }^{1} \mathrm{H} \mathrm{NMR}\left(400 \mathrm{MHz}, \mathrm{CDCl}_{3}\right)$ syn isomer $: \delta 7.26-$ $7.22(\mathrm{~m}, 1 \mathrm{H}), 6.91-6.86(\mathrm{~m}, 2 \mathrm{H}), 6.83-6.78(\mathrm{~m}, 1 \mathrm{H}), 5.85-5.72(\mathrm{~m}, 1 \mathrm{H}), 5.21-5.03(\mathrm{~m}$, $2 \mathrm{H}), 4.57$ (d, $J=5.2 \mathrm{~Hz}, 1 \mathrm{H}), 3.80(\mathrm{~s}, 3 \mathrm{H}), 2.61-2.53(\mathrm{~m}, 1 \mathrm{H}), 1.99$ (brs, $1 \mathrm{H}), 1.01$ (d, $J=$ $6.8 \mathrm{~Hz}, 3 \mathrm{H})$; anti isomer: $4.33(\mathrm{~d}, J=7.6 \mathrm{~Hz}, 1 \mathrm{H}), 3.81(\mathrm{~s}, 3 \mathrm{H}), 2.52-2.43(\mathrm{~m}, 1 \mathrm{H}), 2.16$ (brs, $1 \mathrm{H}), 0.88(\mathrm{~d}, J=6.8 \mathrm{~Hz}, 3 \mathrm{H}) .{ }^{13} \mathrm{C} \mathrm{NMR}\left(100 \mathrm{MHz}, \mathrm{CDCl}_{3}\right)$ syn isomer: $\delta 159.5,144.4,140.3$, 129.0, 118.9, 115.4, 112.7, 112.2, 77.2, 55.2, 44.5, 13.9; anti isomer: $\delta$ 159.6, 144.2, 140.6, $129.2,119.3,116.7,113.1,112.3,77.8,55.2,46.1,16.5$.

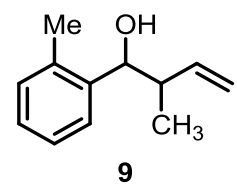

2-Methyl-1-(o-tolyl)but-3-en-1-ol (9). ${ }^{8}$ Synthesized according to the general procedure with 2-methylbenzaldehyde $(0.2 \mathrm{mmol}, 24.0 \mathrm{mg})$ and 1,3-butadiene $(0.4 \mathrm{mmol}, 0.2 \mathrm{ml}, 2 \mathrm{M}$ in THF solution) irradiated with blue LED for $16 \mathrm{~h}$. Purified by flash column chromatography on 
silica gel (PE : DCM = 3: 1) to give 9 as a colourless oil (32.0 $\mathrm{mg}, 91 \%$ yield). syn isomer : anti isomer $=71: 29 .{ }^{1} \mathrm{H}$ NMR $\left(400 \mathrm{MHz}, \mathrm{CDCl}_{3}\right)$ syn isomer: $\delta 7.46-7.39(\mathrm{~m}, 1 \mathrm{H}), 7.23-$ $7.11(\mathrm{~m}, 3 \mathrm{H}), 5.90-5.77(\mathrm{~m}, 1 \mathrm{H}), 5.22-5.01(\mathrm{~m}, 2 \mathrm{H}), 4.84(\mathrm{~d}, J=5.6 \mathrm{~Hz}, 1 \mathrm{H}), 2.60-2.51$ $(\mathrm{m}, 1 \mathrm{H}), 2.31(\mathrm{~s}, 3 \mathrm{H}), 1.81(\mathrm{brs}, 1 \mathrm{H}), 1.06(\mathrm{~d}, J=6.8 \mathrm{~Hz}, 3 \mathrm{H})$; anti isomer: $4.67(\mathrm{~d}, J=7.6 \mathrm{~Hz}$, $1 \mathrm{H}), 2.36(\mathrm{~s}, 3 \mathrm{H}), 2.04$ (brs, $1 \mathrm{H}), 0.91(\mathrm{~d}, J=6.8 \mathrm{~Hz}, 3 \mathrm{H}) .{ }^{13} \mathrm{C} \mathrm{NMR}\left(100 \mathrm{MHz}, \mathrm{CDCl}_{3}\right)$ syn isomer: $\delta 141.1,140.8,134.6,130.3,127.1,126.2,125.9,115.0,73.4,43.2,19.3,13.4$; anti isomer: $\delta 141.1,140.6,135.3,130.3,127.2,126.4,126.1,116.8,73.5,45.7,19.5,16.5$.

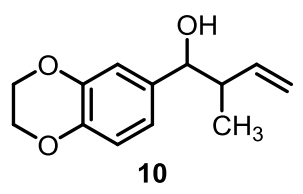

1-(2,3-Dihydrobenzo[b][1,4]dioxin-6-yl)-2-methylbut-3-en-1-ol $\quad$ (10). Synthesized according to the general procedure with 2,3-dihydrobenzo[ $b][1,4]$ dioxine-6-carbaldehyde $(0.2$ mmol, $32.8 \mathrm{mg})$ and 1,3-butadiene $(0.4 \mathrm{mmol}, 0.2 \mathrm{ml}, 2 \mathrm{M}$ in THF solution) irradiated with blue LED for $16 \mathrm{~h}$. Purified by flash column chromatography on silica gel (PE : EA = $10: 1$ ) to give $\mathbf{1 0}$ as a yellow oil ( $42.3 \mathrm{mg}, 96 \%$ yield). The relative configuration of $\mathbf{1 0}$ is determined by comparing the chemical shift of the allyl- $\mathrm{CH}_{3}$ in the ${ }^{1} \mathrm{H}$ and ${ }^{13} \mathrm{C}$ NMR. The chemical shift of syn isomer $(1.01 \mathrm{ppm})$ is in downfield and anti isomer $(0.86 \mathrm{ppm})$ is in upfield (close to $0 \mathrm{ppm}$ ). Furthermore, in the ${ }^{13} \mathrm{C}$ NMR, syn isomer of methyl group in allyl- $\mathrm{CH}_{3}(14.3 \mathrm{ppm})$ is in the upfield and anti isomer $(16.5 \mathrm{ppm})$ is in the downfield. syn isomer : anti isomer $=63: 37 .{ }^{1} \mathrm{H} \mathrm{NMR}\left(400 \mathrm{MHz}, \mathrm{CDCl}_{3}\right)$ syn isomer: $\delta 6.85-6.75(\mathrm{~m}, 3 \mathrm{H})$, $5.85-5.69(\mathrm{~m}, 1 \mathrm{H}), 5.21-5.02(\mathrm{~m}, 2 \mathrm{H}), 4.47(\mathrm{~d}, J=5.2 \mathrm{~Hz}, 1 \mathrm{H}), 4.24(\mathrm{~s}, 4 \mathrm{H}), 2.59-2.48$ (m, 1H), 1.96 (brs, $1 \mathrm{H}), 1.01$ (d, $J=6.8 \mathrm{~Hz}, 3 \mathrm{H})$; anti isomer: $2.45-2.38(\mathrm{~m}, 1 \mathrm{H}), 2.14$ (brs, $1 \mathrm{H}), 0.86(\mathrm{~d}, J=6.8 \mathrm{~Hz}, 3 \mathrm{H}) .{ }^{13} \mathrm{C} \mathrm{NMR}\left(100 \mathrm{MHz}, \mathrm{CDCl}_{3}\right)$ syn isomer: $\delta 143.1,142.7,140.3$, $136.1,119.5,116.7,115.6,115.4,76.9,64.3,44.5,14.3$; anti isomer: $\delta 143.3,143.0,140.8$, 135.9, 119.9, 116.9, 116.6, 115.3, 77.4, 64.3, 46.1, 16.5. HRMS (ESI) Calcd for $\mathrm{C}_{13} \mathrm{H}_{16} \mathrm{O}_{3} \mathrm{Na}$ $[\mathrm{M}+\mathrm{Na}]^{+}$243.0997, Found 243.0993.

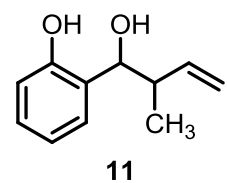

2-(1-Hydroxy-2-methylbut-3-en-1-yl)phenol (11). ${ }^{6}$ Synthesized according to the general procedure with 2-hydroxybenzaldehyde $(0.2 \mathrm{mmol}, 24.4 \mathrm{mg})$ and 1,3-butadiene $(0.4 \mathrm{mmol}$, $0.2 \mathrm{ml}, 2 \mathrm{M}$ in THF solution) irradiated with blue LED for $16 \mathrm{~h}$. Purified by flash column chromatography on silica gel (PE : EA $=10: 1)$ to give $\mathbf{1 1}$ as a colourless oil $(31.7 \mathrm{mg}, 89 \%$ yield). syn isomer : anti isomer $=38: 63 .{ }^{1} \mathrm{H} \mathrm{NMR}\left(400 \mathrm{MHz}, \mathrm{CDCl}_{3}\right)$ syn isomer: $\delta 7.97$ (brs, 1H), $7.20-7.11(\mathrm{~m}, 1 \mathrm{H}), 6.95-6.79(\mathrm{~m}, 3 \mathrm{H}), 5.84-5.75(\mathrm{~m}, 1 \mathrm{H}), 5.28-5.08(\mathrm{~m}, 2 \mathrm{H}), 4.78$ $(\mathrm{d}, J=5.2 \mathrm{~Hz}, 1 \mathrm{H}), 2.91(\mathrm{brs}, 1 \mathrm{H}), 2.72-2.60(\mathrm{~m}, 1 \mathrm{H}), 1.09(\mathrm{~d}, J=6.8 \mathrm{~Hz}, 3 \mathrm{H})$; anti isomer: $4.44(\mathrm{~d}, J=8.8 \mathrm{~Hz}, 1 \mathrm{H}), 0.90(\mathrm{~d}, J=6.8 \mathrm{~Hz}, 3 \mathrm{H}) .{ }^{13} \mathrm{C} \mathrm{NMR}\left(100 \mathrm{MHz}, \mathrm{CDCl}_{3}\right)$ syn isomer: $\delta$ $155.9,139.9,129.1,128.7,128.0,118.0,117.1,116.1,79.3,44.4,13.4$; anti isomer: $\delta$ 155.6, $140.3,129.1,128.9,128.0,119.4,117.3,116.1,79.4,44.7,16.9$. 


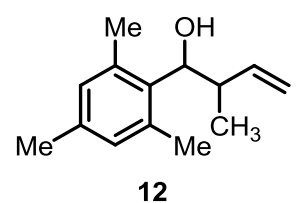

1-Mesityl-2-methylbut-3-en-1-ol (12). ${ }^{9}$ Synthesized according to the general procedure with 2,4,6-trimethylbenzaldehyde $(0.2 \mathrm{mmol}, 29.6 \mathrm{mg})$ and 1,3-butadiene $(0.4 \mathrm{mmol}, 0.2 \mathrm{ml}, 2 \mathrm{M}$ in THF solution) irradiated with blue LED for $16 \mathrm{~h}$. Purified by flash column chromatography on silica gel (PE : $\mathrm{EA}=10: 1)$ to give $\mathbf{1 2}$ as a colourless oil $(24.0 \mathrm{mg}, 59 \%$ yield). syn isomer : anti isomer $=42: 58 .{ }^{1} \mathrm{H}$ NMR $\left(400 \mathrm{MHz}, \mathrm{CDCl}_{3}\right)$ syn isomer: $6.79(\mathrm{~s}, 2 \mathrm{H}), 5.51$ (ddd, $J=17.6,10.8,7.2 \mathrm{~Hz}, 1 \mathrm{H}), 4.94-4.81(\mathrm{~m}, 2 \mathrm{H}), 4.75-4.81(\mathrm{~m}, 1 \mathrm{H}), 2.94-2.83(\mathrm{~m}, 1 \mathrm{H})$, $2.37(\mathrm{~s}, 6 \mathrm{H}), 2.23(\mathrm{~s}, 3 \mathrm{H}), 2.23(\mathrm{~s}, 1 \mathrm{H}), 1.78(\mathrm{brs}, 1 \mathrm{H}), 1.26(\mathrm{~d}, J=6.4 \mathrm{~Hz}, 3 \mathrm{H})$; anti isomer: $\delta$ $6.81(\mathrm{~s}, 2 \mathrm{H}), 5.89(\mathrm{ddd}, J=17.2,10.4,8.4 \mathrm{~Hz}, 1 \mathrm{H}), 5.27-5.18(\mathrm{~m}, 2 \mathrm{H}), 2.41(\mathrm{~s}, 6 \mathrm{H}), 2.24(\mathrm{~s}$, $3 \mathrm{H}), 1.96$ (brs, $1 \mathrm{H}), 0.79$ (d, $J=7.2 \mathrm{~Hz}, 3 \mathrm{H}) .{ }^{13} \mathrm{C} \mathrm{NMR}\left(100 \mathrm{MHz}, \mathrm{CDCl}_{3}\right.$ ) syn isomer: $\delta$ $140.0,136.6,136.5,135.8,130.2,114.2,75.3,42.1,21.0,21.0,20.7,16.5$; anti isomer: $\delta$ $142.2,136.8,136.3,134.5,130.1,116.4,74.0,43.8,21.0,21.0,20.7,16.7$.

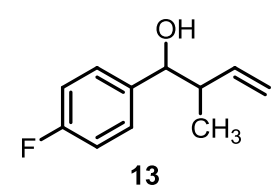

1-(4-Fluorophenyl)-2-methylbut-3-en-1-ol (13). ${ }^{10}$ Synthesized according to the general procedure with 4-fluorobenzaldehyde $(0.2 \mathrm{mmol}, 24.8 \mathrm{mg})$ and 1,3-butadiene $(0.4 \mathrm{mmol}, 0.2$ $\mathrm{ml}, 2 \mathrm{M}$ in THF solution) irradiated with blue LED for $16 \mathrm{~h}$. Purified by flash column chromatography on silica gel (PE : EA $=10: 1)$ to give $\mathbf{1 3}$ as a colourless oil $(32.0 \mathrm{mg}, 89 \%$ yield). syn isomer : anti isomer $=67: 33 .{ }^{1} \mathrm{H}$ NMR $\left(400 \mathrm{MHz}, \mathrm{CDCl}_{3}\right)$ syn isomer: $\delta 7.30-$ $7.23(\mathrm{~m}, 2 \mathrm{H}), 7.04-6.97(\mathrm{~m}, 2 \mathrm{H}), 5.82-5.67(\mathrm{~m}, 1 \mathrm{H}), 5.20-5.00(\mathrm{~m}, 2 \mathrm{H}), 4.56(\mathrm{~d}, J=4.8$ $\mathrm{Hz}, 1 \mathrm{H}), 2.57-2.48(\mathrm{~m}, 1 \mathrm{H}), 2.30$ (brs, $1 \mathrm{H}), 0.99(\mathrm{~d}, J=6.8 \mathrm{~Hz}, 3 \mathrm{H})$; anti isomer: 4.32 (d, $J$ $=8.0 \mathrm{~Hz}, 1 \mathrm{H}), \delta 2.46-2.37(\mathrm{~m}, 1 \mathrm{H}), 2.14(\mathrm{brs}, 1 \mathrm{H}), 0.85(\mathrm{~d}, J=6.8 \mathrm{~Hz}, 3 \mathrm{H}) .{ }^{13} \mathrm{C}$ NMR $(100$ $\left.\mathrm{MHz}, \mathrm{CDCl}_{3}\right)$ syn isomer: $\delta 162.0(\mathrm{~d}, J=244.0 \mathrm{~Hz}), 140.0,138.3(\mathrm{~d}, J=3.0 \mathrm{~Hz}) 128.0(\mathrm{~d}, J=$ $8.0 \mathrm{~Hz}), 115.7,114.8(\mathrm{~d}, J=21.0 \mathrm{~Hz}), 76.7,44.7,14.0$; anti isomer: $\delta 162.2(\mathrm{~d}, J=244.0 \mathrm{~Hz})$, 140.4, $138.1(\mathrm{~d}, J=3.0 \mathrm{~Hz}), 128.4(\mathrm{~d}, J=8.0 \mathrm{~Hz}), 117.0,115.0(\mathrm{~d}, J=21.0 \mathrm{~Hz}), 77.2,46.4$, 16.4. ${ }^{19} \mathrm{~F}$ NMR $\left(377 \mathrm{MHz}, \mathrm{CDCl}_{3}\right) \delta-115.04,-115.45$.

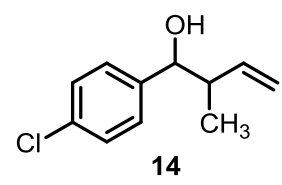

1-(4-Chlorophenyl)-2-methylbut-3-en-1-ol (14). ${ }^{11}$ Synthesized according to the general procedure with 4-chlorobenzaldehyde $(0.2 \mathrm{mmol}, 28.0 \mathrm{mg})$ and 1,3-butadiene $(0.4 \mathrm{mmol}, 0.2$ $\mathrm{ml}, 2 \mathrm{M}$ in THF solution) irradiated with blue LED for $16 \mathrm{~h}$. Purified by flash column chromatography on silica gel (PE : DCM $=3: 1)$ to give 14 as a pale yellow oil $(34.5 \mathrm{mg}, 88 \%$ yield). syn isomer : anti isomer $=59: 41 .{ }^{1} \mathrm{H}$ NMR $\left(400 \mathrm{MHz}, \mathrm{CDCl}_{3}\right)$ syn isomer: $\delta 7.33-$ $7.22(\mathrm{~m}, 4 \mathrm{H}), 5.81-5.69(\mathrm{~m}, 1 \mathrm{H}), 5.20-5.02(\mathrm{~m}, 2 \mathrm{H}), 4.59(\mathrm{~d}, J=5.2 \mathrm{~Hz}, 1 \mathrm{H}), 2.58-2.50$ (m, 1H), 2.02 (brs, $1 \mathrm{H}), 0.98(\mathrm{~d}, J=6.8 \mathrm{~Hz}, 3 \mathrm{H})$; anti isomer: $4.34(\mathrm{~d}, J=8.0 \mathrm{~Hz}, 1 \mathrm{H}), 2.47$ - 
$2.38(\mathrm{~m}, 1 \mathrm{H}), 2.21(\mathrm{brs}, 1 \mathrm{H}), 0.87(\mathrm{~d}, J=6.8 \mathrm{~Hz}, 3 \mathrm{H}) .{ }^{13} \mathrm{C}$ NMR $\left(100 \mathrm{MHz}, \mathrm{CDCl}_{3}\right)$ syn isomer: $\delta 141.0,139.9,133.0,128.2,127.9,115.9,76.5,44.6,13.8$; anti isomer: $\delta 140.9$, $140.2,133.3,128.4,127.9,117.2,77.1,46.3,16.4$.

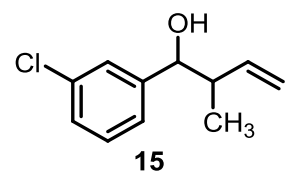

1-(3-Chlorophenyl)-2-methylbut-3-en-1-ol (15). ${ }^{12}$ Synthesized according to the general procedure with 3-chlorobenzaldehyde $(0.2 \mathrm{mmol}, 28.0 \mathrm{mg})$ and 1,3-butadiene ( $0.4 \mathrm{mmol}, 0.2$ $\mathrm{ml}, 2 \mathrm{M}$ in THF solution) irradiated with blue LED for $16 \mathrm{~h}$. Purified by flash column chromatography on silica gel (PE : DCM $=3: 1)$ to give 15 as a pale yellow oil (19.6 $\mathrm{mg}, 50 \%$ yield). syn isomer : anti isomer $=63: 37 .{ }^{1} \mathrm{H} \mathrm{NMR}\left(400 \mathrm{MHz}, \mathrm{CDCl}_{3}\right)$ syn isomer: $\delta 7.33-$ $7.15(\mathrm{~m}, 4 \mathrm{H}), 5.80-5.70(\mathrm{~m}, 1 \mathrm{H}), 5.20-5.03(\mathrm{~m}, 2 \mathrm{H}), 4.57(\mathrm{~d}, J=4.0 \mathrm{~Hz}, 1 \mathrm{H}), 2.58-2.50$ (m, 1H), 2.16 (brs, 1H), $0.98(\mathrm{~d}, J=6.8 \mathrm{~Hz}, 3 \mathrm{H})$; anti isomer: $4.32(\mathrm{~d}, J=6.8 \mathrm{~Hz}, 1 \mathrm{H}), 2.48-$ $2.39(\mathrm{~m}, 1 \mathrm{H}), 2.31$ (brs, $1 \mathrm{H}), 0.88(\mathrm{~d}, J=6.8 \mathrm{~Hz}, 3 \mathrm{H}) .{ }^{13} \mathrm{C}$ NMR $\left(100 \mathrm{MHz}, \mathrm{CDCl}_{3}\right)$ syn isomer: $\delta 144.7,139.8,134.0,129.3,127.4,126.6,124.6,115.9,76.5,44.5,13.7$; anti isomer: $\delta 144.5,140.0,134.1,129.4,127.7,126.9,125.0,117.2,77.1,46.2,16.4$.

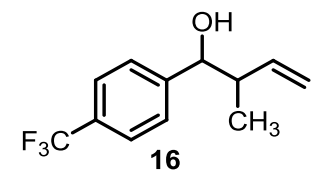

2-Methyl-1-(4-(trifluoromethyl)phenyl)but-3-en-1-ol (16). ${ }^{13}$ Synthesized according to the general procedure with 4-(trifluoromethyl)benzaldehyde $(0.2 \mathrm{mmol}, 34.8 \mathrm{mg})$ and 1,3-butadiene $(0.4 \mathrm{mmol}, 0.2 \mathrm{ml}, 2 \mathrm{M}$ in THF solution) irradiated with blue LED for $16 \mathrm{~h}$. Purified by flash column chromatography on silica gel (PE : DCM $=3: 1)$ to give $\mathbf{1 6}$ as a pale yellow oil (15.6 mg, 34\% yield). syn isomer : anti isomer $=60: 40 .{ }^{1} \mathrm{H} \mathrm{NMR}(400 \mathrm{MHz}$, $\left.\mathrm{CDCl}_{3}\right)$ syn isomer: $\delta 7.61-7.58(\mathrm{~m}, 2 \mathrm{H}), 7.44(\mathrm{~m}, 2 \mathrm{H}), 5.81-5.72(\mathrm{~m}, 1 \mathrm{H}), 5.22-5.05(\mathrm{~m}$, $2 \mathrm{H}), 4.70(\mathrm{~d}, J=3.6 \mathrm{~Hz}, 1 \mathrm{H}), 2.63-2.53(\mathrm{~m}, 1 \mathrm{H}), 2.06$ (brs, 1H), $0.98(\mathrm{~d}, J=7.2 \mathrm{~Hz}, 3 \mathrm{H})$; anti isomer: $4.44(\mathrm{~d}, J=7.6 \mathrm{~Hz}, 1 \mathrm{H}), 2.51-2.42(\mathrm{~m}, 1 \mathrm{H}), 2.25$ (brs, $1 \mathrm{H}), 0.90(\mathrm{~d}, J=6.8 \mathrm{~Hz}$, $3 \mathrm{H}) .{ }^{13} \mathrm{C} \mathrm{NMR}\left(100 \mathrm{MHz}, \mathrm{CDCl}_{3}\right)$ syn isomer: $\delta 146.5,139.7,129.8(\mathrm{q}, J=29.0 \mathrm{~Hz}), 126.8$, $125.0(\mathrm{q}, J=4.0 \mathrm{~Hz}), 124.2(\mathrm{q}, J=270.0 \mathrm{~Hz}), 116.2,76.5,44.6,13.5$; anti isomer: $\delta 146.4$, 139.8, $129.8(\mathrm{q}, J=29.0 \mathrm{~Hz}), 127.1,125.1(\mathrm{q}, J=4.0 \mathrm{~Hz}), 124.2(\mathrm{q}, J=270.0 \mathrm{~Hz}), 117.5$, 77.2, 46.3, 16.3. ${ }^{19} \mathrm{~F}$ NMR $\left(377 \mathrm{MHz}, \mathrm{CDCl}_{3}\right) \delta-62.44,-62.48$.

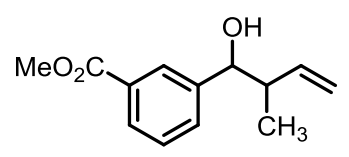

17

Methyl 3-(1-hydroxy-2-methylbut-3-en-1-yl)benzoate (17). Synthesized according to the general procedure with methyl 3-formylbenzoate $(0.2 \mathrm{mmol}, 32.8 \mathrm{mg})$ and 1,3-butadiene $(0.4$ mmol, $0.2 \mathrm{ml}, 2 \mathrm{M}$ in THF solution) irradiated with blue LED for $16 \mathrm{~h}$. Purified by flash column chromatography on silica gel (PE : EA $=10: 1)$ to give $\mathbf{1 7}$ as a pale yellow oil (34.7 
$\mathrm{mg}, 79 \%$ yield). The relative configuration of 17 is determined according to the same method as 6. syn isomer : anti isomer $=59: 41 .{ }^{1} \mathrm{H} \mathrm{NMR}\left(400 \mathrm{MHz}, \mathrm{CDCl}_{3}\right)$ syn isomer: $\delta 8.01-$ $7.30(\mathrm{~m}, 2 \mathrm{H}), 7.55-7.51(\mathrm{~m}, 1 \mathrm{H}), 7.44-7.40(\mathrm{~m}, 1 \mathrm{H}), 5.83-5.72(\mathrm{~m}, 1 \mathrm{H}), 5.22-5.04(\mathrm{~m}$, 2H), 4.67 (d, $J=4.8 \mathrm{~Hz}, 1 \mathrm{H}), 3.92$ (s, 3H), $2.64-2.56$ (m, 1H), 2.13 (brs, 1H), 0.99 (d, $J=$ $6.8 \mathrm{~Hz}, 3 \mathrm{H})$; anti isomer: $4.43(\mathrm{~d}, J=7.6 \mathrm{~Hz}, 1 \mathrm{H}), 2.54-2.45(\mathrm{~m}, 1 \mathrm{H}), 2.31$ (brs, $1 \mathrm{H}), 0.89$ (d, $J=6.8 \mathrm{~Hz}, 3 \mathrm{H}) .{ }^{13} \mathrm{C} \mathrm{NMR}\left(100 \mathrm{MHz}, \mathrm{CDCl}_{3}\right)$ syn isomer: $\delta 167.1,143.0,139.9,131.0,129.9$, 128.5, 128.1, 127.6, 115.9, 76.7, 52.1, 44.6, 13.7; anti isomer: $\delta$ 167.0, 142.9, 140.0, 131.4, 130.1, 128.8, 128.3, 128.0, 117.2, 77.3, 52.1, 46.3, 16.4. HRMS (ESI) Calcd for $\mathrm{C}_{13} \mathrm{H}_{16} \mathrm{O}_{3} \mathrm{Na}$ $[\mathrm{M}+\mathrm{Na}]^{+}$243.0997, Found 243.0993.

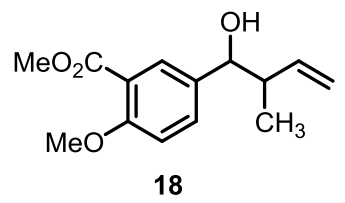

Methyl 5-(1-hydroxy-2-methylbut-3-en-1-yl)-2-methoxybenzoate (18). Synthesized according to the general procedure with methyl 4-formyl-2-methoxybenzoate $(0.2 \mathrm{mmol}, 38.8$ $\mathrm{mg}$ ) and 1,3-butadiene ( $0.4 \mathrm{mmol}, 0.2 \mathrm{ml}, 2 \mathrm{M}$ in THF solution) irradiated with blue LED for $16 \mathrm{~h}$. Purified by flash column chromatography on silica gel (PE : EA $=10: 1)$ to give $\mathbf{1 8}$ as a pale yellow oil (49.5 $\mathrm{mg}$, 99\% yield). The relative configuration of $\mathbf{1 8}$ is determined according to the same method as 6. $s y n$ isomer : anti isomer $=66: 34 .{ }^{1} \mathrm{H}$ NMR $(400 \mathrm{MHz}$, $\left.\mathrm{CDCl}_{3}\right)$ syn isomer: $\delta 7.76-7.70(\mathrm{~m}, 1 \mathrm{H}), 7.45-7.40(\mathrm{~m}, 1 \mathrm{H}), 6.96-6.92(\mathrm{~m}, 1 \mathrm{H}), 5.90-$ $5.61(\mathrm{~m}, 1 \mathrm{H}), 5.11(\mathrm{~m}, 2 \mathrm{H}), 4.57(\mathrm{~d}, J=5.6 \mathrm{~Hz}, 1 \mathrm{H}), 3.89(\mathrm{~s}, 3 \mathrm{H}), 3.88(\mathrm{~s}, 3 \mathrm{H}), 2.59-2.51$ $(\mathrm{m}, 1 \mathrm{H}), 2.21(\mathrm{brs}, 1 \mathrm{H}), 1.00(\mathrm{~d}, J=6.8 \mathrm{~Hz}, 3 \mathrm{H})$; anti isomer: $4.33(\mathrm{~d}, J=8.0 \mathrm{~Hz}, 1 \mathrm{H}), 3.90(\mathrm{~s}$, $3 \mathrm{H}), 3.88(\mathrm{~s}, 3 \mathrm{H}), 2.49-2.40(\mathrm{~m}, 1 \mathrm{H}), 2.34$ (brs, $1 \mathrm{H}), 0.86(\mathrm{~d}, J=6.8 \mathrm{~Hz}, 3 \mathrm{H}) .{ }^{13} \mathrm{C} \mathrm{NMR}$ $\left(100 \mathrm{MHz}, \mathrm{CDCl}_{3}\right)$ syn isomer: $\delta 166.6,158.3,140.0,134.4,131.6,129.7,119.3,115.7,111.6$, 76.4, 56.0, 52.0, 44.6, 14.1; anti isomer: $\delta$ 166.6, 158.6, 140.4, 134.2, 131.9, 130.1, 119.5, 117.0, 111.8, 76.9, 56.0, 52.0, 46.3, 16.4. HRMS (ESI) Calcd for $\mathrm{C}_{14} \mathrm{H}_{18} \mathrm{O}_{4} \mathrm{Na}[\mathrm{M}+\mathrm{Na}]^{+}$ 273.1103, Found 273.1103.

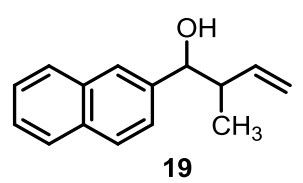

2-Methyl-1-(naphthalen-2-yl)but-3-en-1-ol (19). ${ }^{13}$ Synthesized according to the general procedure with 2-naphthaldehyde $(0.2 \mathrm{mmol}, 31.2 \mathrm{mg})$ and 1,3-butadiene $(0.4 \mathrm{mmol}, 0.2 \mathrm{ml}$, $2 \mathrm{M}$ in THF solution) irradiated with blue LED for $16 \mathrm{~h}$. Purified by flash column chromatography on silica gel (PE : EA $=10: 1)$ to give 19 as a pale yellow oil $(36.8 \mathrm{mg}, 87 \%$ yield). syn isomer : anti isomer $=56: 43 .{ }^{1} \mathrm{H} \mathrm{NMR}\left(400 \mathrm{MHz}, \mathrm{CDCl}_{3}\right)$ syn isomer: $\delta 7.83-$ $7.74(\mathrm{~m}, 4 \mathrm{H}), 7.48-7.40(\mathrm{~m}, 3 \mathrm{H}), 5.88-5.75(\mathrm{~m}, 1 \mathrm{H}), 5.23-5.03(\mathrm{~m}, 2 \mathrm{H}), 4.76(\mathrm{~d}, J=5.6$ $\mathrm{Hz}, 1 \mathrm{H}), 2.72-2.63(\mathrm{~m}, 1 \mathrm{H}), 2.08$ (brs, $1 \mathrm{H}), 1.03(\mathrm{~d}, J=6.8 \mathrm{~Hz}, 3 \mathrm{H})$; anti isomer: 4.51 (d, $J$ $=7.6 \mathrm{~Hz}, 1 \mathrm{H}), 2.63-2.54(\mathrm{~m}, 1 \mathrm{H}), 2.26(\mathrm{brs}, 1 \mathrm{H}), 0.89(\mathrm{~d}, J=6.8 \mathrm{~Hz}, 3 \mathrm{H}) .{ }^{13} \mathrm{C}$ NMR $(100$ $\mathrm{MHz}, \mathrm{CDCl}_{3}$ ) syn isomer: $\delta 140.3,140.1,133.1,132.9,128.0,127.9,127.6,126.0,125.8$, 125.3, 124.6, 115.6, 77.4, 44.6, 14.0; anti isomer: $\delta 140.5,139.9,133.2,133.1,128.0,127.8$, $127.7,126.0,125.9,125.7,124.6,116.9,78.0,46.1,16.6$. 


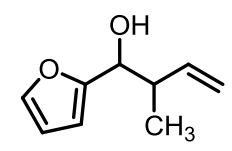

20

1-(Furan-2-yl)-2-methylbut-3-en-1-ol (20). ${ }^{13}$ Synthesized according to the general procedure with furan-2-carbaldehyde $(0.2 \mathrm{mmol}, 19.2 \mathrm{mg})$ and 1,3-butadiene $(0.4 \mathrm{mmol}, 0.2$ $\mathrm{ml}, 2 \mathrm{M}$ in THF solution) irradiated with blue LED for $16 \mathrm{~h}$. Purified by flash column chromatography on silica gel (PE : DCM $=1: 1)$ to give $\mathbf{2 0}$ as a yellow oil $(16.7 \mathrm{mg}, 55 \%$ yield). syn isomer : anti isomer $=56: 43 .{ }^{1} \mathrm{H}$ NMR $\left(400 \mathrm{MHz}, \mathrm{CDCl}_{3}\right)$ syn isomer: $\delta 7.38-$ $7.35(\mathrm{~m}, 1 \mathrm{H}), 6.34-6.31(\mathrm{~m}, 1 \mathrm{H}), 6.27-6.22(\mathrm{~m}, 1 \mathrm{H}), 5.85-5.71(\mathrm{~m}, 1 \mathrm{H}), 5.23-5.04(\mathrm{~m}$, $2 \mathrm{H}), 4.56(\mathrm{~d}, J=4.8 \mathrm{~Hz}, 1 \mathrm{H}), 2.75-2.66(\mathrm{~m}, 1 \mathrm{H}), 2.12(\mathrm{~d}, J=4.8 \mathrm{~Hz}, 1 \mathrm{H}), 1.07(\mathrm{~d}, J=6.8$ $\mathrm{Hz}, 3 \mathrm{H})$; anti isomer: $4.42(\mathrm{dd}, J=2.8,7.6 \mathrm{~Hz}, 1 \mathrm{H}), 2.17(\mathrm{~d}, J=3.6 \mathrm{~Hz}, 1 \mathrm{H}), 0.95(\mathrm{~d}, J=6.8$ $\mathrm{Hz}, 3 \mathrm{H}) .{ }^{13} \mathrm{C} \mathrm{NMR}\left(100 \mathrm{MHz}, \mathrm{CDCl}_{3}\right)$ syn isomer: $\delta 155.4,141.7,139.6,115.9,110.0,106.8$, 71.4, 43.1, 15.0; anti isomer: $\delta 155.1,142.0,140.0,116.9,110.1,107.2,71.4,43.6,16.2$.

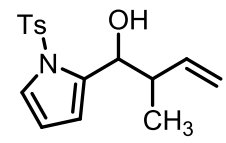

21

2-Methyl-1-(1-tosyl-1H-pyrrol-2-yl)but-3-en-1-ol (21). Synthesized according to the general procedure with 1-tosyl-1H-pyrrole-2-carbaldehyde $(0.2 \mathrm{mmol}, 49.8 \mathrm{mg})$ and 1,3-butadiene ( $0.4 \mathrm{mmol}, 0.2 \mathrm{ml}, 2 \mathrm{M}$ in THF solution) irradiated with blue LED for $16 \mathrm{~h}$. Purified by flash column chromatography on silica gel (PE : DCM $=1: 1)$ to give $\mathbf{2 1}$ as a pale yellow oil (42.7 mg, 70\% yield). The relative configuration of $\mathbf{2 1}$ is determined according to the same method as 10. syn isomer : anti isomer $=82: 18 .{ }^{1} \mathrm{H}$ NMR $\left(400 \mathrm{MHz}, \mathrm{CDCl}_{3}\right)$ syn isomer: $\delta 7.69-7.63(\mathrm{~m}, 2 \mathrm{H}), 7.29-7.26(\mathrm{~m}, 3 \mathrm{H}), 6.31-6.29(\mathrm{~m}, 1 \mathrm{H}), 6.26-6.23(\mathrm{~m}, 1 \mathrm{H})$, $5.62(\mathrm{ddd}, J=17.2,10.4,6.8 \mathrm{~Hz}, 1 \mathrm{H}), 4.98-4.89(\mathrm{~m}, 2 \mathrm{H}), 4.81-4.77(\mathrm{~m}, 1 \mathrm{H}), 2.76-2.63$ $(\mathrm{m}, 1 \mathrm{H}), 2.40(\mathrm{~s}, 3 \mathrm{H}), 1.07(\mathrm{~d}, J=6.8 \mathrm{~Hz}, 3 \mathrm{H})$; anti isomer: $5.90-5.81(\mathrm{~m}, 1 \mathrm{H}), 5.15-5.10$ $(\mathrm{m}, 2 \mathrm{H}), 2.39(\mathrm{~s}, 3 \mathrm{H}), 0.89(\mathrm{~d}, J=6.8 \mathrm{~Hz}, 3 \mathrm{H}) .{ }^{13} \mathrm{C} \mathrm{NMR}\left(100 \mathrm{MHz}, \mathrm{CDCl}_{3}\right)$ syn isomer: $\delta$ 145.0, 140.5, 136.8, 136.4, 130.0, 126.6, 123.2, 114.9, 113.7, 111.6, 69.4, 42.2, 21.5, 15.2; anti isomer: $\delta 145.0,140.1,136.9,136.5,129.9,126.7,123.4,116.4,113.1,111.7,69.3,43.8$, 21.5, 17.3. HRMS (ESI) Calcd for $\mathrm{C}_{16} \mathrm{H}_{19} \mathrm{NO}_{3} \mathrm{SNa}[\mathrm{M}+\mathrm{Na}]^{+}$328.0983, Found 328.0987.

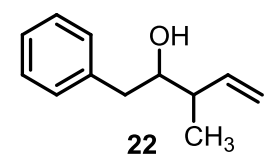

3-Methyl-1-phenylpent-4-en-2-ol (22). ${ }^{14}$ Synthesized according to the general procedure with 2-phenylacetaldehyde $(0.2 \mathrm{mmol}, 24.0 \mathrm{mg})$ and 1,3-butadiene $(0.4 \mathrm{mmol}, 0.2 \mathrm{ml}, 2 \mathrm{M}$ in THF solution) irradiated with blue LED for $16 \mathrm{~h}$. Purified by flash column chromatography on silica gel (PE : DCM = 2:1) to give 22 as a colourless oil $(25.7 \mathrm{mg}, 73 \%$ yield). syn isomer : anti isomer $=55: 45 .{ }^{1} \mathrm{H}$ NMR $\left(400 \mathrm{MHz}, \mathrm{CDCl}_{3}\right) \delta 7.32-7.28(\mathrm{~m}, 2 \mathrm{H}), 7.24-7.20$ $(\mathrm{m}, 3 \mathrm{H}), 5.91-5.82(\mathrm{~m}, 1 \mathrm{H}), 5.16-5.10(\mathrm{~m}, 2 \mathrm{H}), 3.73-3.66(\mathrm{~m}, 1 \mathrm{H}), 2.90-2.83(\mathrm{~m}, 1 \mathrm{H})$, $2.66-2.57$ (m, 1H), $2.37-2.29(\mathrm{~m}, 1 \mathrm{H}), 1.63$ (brs, $1 \mathrm{H}), 1.11(\mathrm{~d}, J=6.8 \mathrm{~Hz}, 3 \mathrm{H}) .{ }^{13} \mathrm{C} \mathrm{NMR}$ 
$\left(100 \mathrm{MHz}, \mathrm{CDCl}_{3}\right)$ syn isomer: $\delta 140.9,139.0,129.3,128.5,126.4,115.3,75.7,43.1,40.8$, 14.6; anti isomer: $\delta 139.9,138.9,129.4,128.5,126.3,116.1,75.8,43.3,40.9,16.4$.

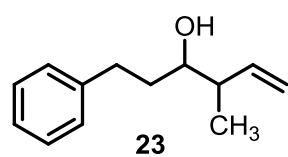

4-Methyl-1-phenylhex-5-en-3-ol (23). ${ }^{5}$ Synthesized according to the general procedure with 3-phenylpropanal $(0.2 \mathrm{mmol}, 26.8 \mathrm{mg})$ and 1,3-butadiene $(0.4 \mathrm{mmol}, 0.2 \mathrm{ml}, 2 \mathrm{M}$ in THF solution) irradiated with blue LED for $16 \mathrm{~h}$. Purified by flash column chromatography on silica gel (PE : DCM = 2:1) to give $\mathbf{2 3}$ as a colourless oil (26.6 $\mathrm{mg}, 70 \%$ yield). syn isomer : anti isomer $=55: 45 .{ }^{1} \mathrm{H}$ NMR $\left(400 \mathrm{MHz}, \mathrm{CDCl}_{3}\right)$ syn isomer: $\delta 7.30-7.25(\mathrm{~m}, 2 \mathrm{H}), 7.22-$ $7.16(\mathrm{~m}, 3 \mathrm{H}), 5.81-5.70(\mathrm{~m}, 1 \mathrm{H}), 5.14-5.06(\mathrm{~m}, 2 \mathrm{H}), 3.54-3.50(\mathrm{~m}, 1 \mathrm{H}), 2.89-2.81(\mathrm{~m}$, $1 \mathrm{H}), 2.71-2.61(\mathrm{~m}, 1 \mathrm{H}), 2.34-2.19(\mathrm{~m}, 1 \mathrm{H}), 1.88-1.78(\mathrm{~m}, 1 \mathrm{H}), 1.74-1.64(\mathrm{~m}, 2 \mathrm{H}), 1.03$ $(\mathrm{d}, J=6.8 \mathrm{~Hz}, 3 \mathrm{H})$; anti isomer: $3.44-3.39(\mathrm{~m}, 1 \mathrm{H}) .{ }^{13} \mathrm{C}$ NMR $\left(100 \mathrm{MHz}, \mathrm{CDCl}_{3}\right)$ syn isomer: $\delta 142.2,140.8,128.4,128.4,125.8,115.4,74.1,43.7,35.8,32.4,14.3$; anti isomer: $\delta$ $142.3,140.2,128.4,128.4,125.8,116.4,74.0,44.3,36.1,32.2,16.2$.

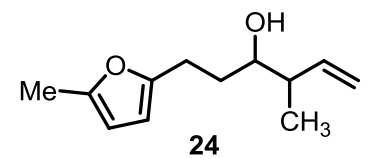

4-Methyl-1-(5-methylfuran-2-yl)hex-5-en-3-ol (24). Synthesized according to the general procedure with 3-(5-methylfuran-2-yl)propanal $(0.2 \mathrm{mmol}, 27.6 \mathrm{mg})$ and 1,3-butadiene $(0.4$ mmol, $0.2 \mathrm{ml}, 2 \mathrm{M}$ in THF solution) irradiated with blue LED for $16 \mathrm{~h}$. Purified by flash column chromatography on silica gel ( $\mathrm{PE}: \mathrm{DCM}=1.1: 1)$ to give $\mathbf{2 4}$ as a colourless oil (30.3 $\mathrm{mg}, \mathbf{7 8 \%}$ yield). The relative configuration of $\mathbf{2 4}$ is determined by comparing the chemical shift of the ortho-OH-(C-H) on the ${ }^{1} \mathrm{H}$ NMR. The chemical shift of syn isomer (3.53 ppm) is in downfield and anti isomer (3.43 ppm) is in upfield. Furthermore, in the ${ }^{13} \mathrm{C}$ NMR, syn isomer at allyl- $\mathrm{CH}_{3}$ is in the upfield $(14.3 \mathrm{ppm})$ and anti isomer $(16.2 \mathrm{ppm})$ is in the downfield. syn isomer : anti isomer $=56: 44 .{ }^{1} \mathrm{H}$ NMR $\left(400 \mathrm{MHz}, \mathrm{CDCl}_{3}\right)$ syn isomer $: \delta 5.87$ $-5.71(\mathrm{~m}, 2 \mathrm{H}), 5.81-5.71(\mathrm{~m}, 1 \mathrm{H}), 5.14-5.07(\mathrm{~m}, 2 \mathrm{H}), 3.53(\mathrm{~m}, 1 \mathrm{H}), 2.82-2.75(\mathrm{~m}, 2 \mathrm{H})$, $2.34-2.18(\mathrm{~m}, 4 \mathrm{H}), 1.71-1.60(\mathrm{~m}, 2 \mathrm{H}), 1.53(\mathrm{brs}, 1 \mathrm{H}), 1.04(\mathrm{~d}, J=6.8 \mathrm{~Hz}, 3 \mathrm{H})$; anti isomer: $3.43(\mathrm{~m}, 1 \mathrm{H}), 2.67-2.60(\mathrm{~m}, 2 \mathrm{H}), 1.91-1.81(\mathrm{~m}, 2 \mathrm{H}), 1.04(\mathrm{~d}, J=6.8 \mathrm{~Hz}, 3 \mathrm{H}) .{ }^{13} \mathrm{C} \mathrm{NMR}$ $\left(100 \mathrm{MHz}, \mathrm{CDCl}_{3}\right)$ syn isomer: $\delta 154.0,150.3,140.7,115.4,105.8,105.5,74.1,43.7,32.6$, 24.8, 14.3, 13.5; anti isomer: $\delta 154.1,150.3,140.2,116.4,105.8,105.5,74.0,44.2,32.8,24.5$, 16.2, 13.5. HRMS (ESI) Calcd for $\mathrm{C}_{12} \mathrm{H}_{19} \mathrm{O}_{2}[\mathrm{M}+\mathrm{H}]^{+}$195.1385, Found 195.1357.

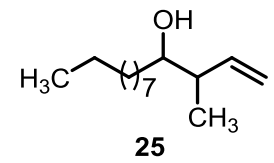

3-Methyltridec-1-en-4-ol (25). ${ }^{15}$ Synthesized according to the general procedure with decanal $(0.2 \mathrm{mmol}, 31.2 \mathrm{mg})$ and 1,3-butadiene $(0.4 \mathrm{mmol}, 0.2 \mathrm{ml}, 2 \mathrm{M}$ in THF solution) irradiated with blue LED for $16 \mathrm{~h}$. Purified by flash column chromatography on silica gel (PE : 
$\mathrm{DCM}=1: 1)$ to give $\mathbf{2 5}$ as a colourless oil ( $34.3 \mathrm{mg}, 81 \%$ yield). syn isomer : anti isomer $=$ 56: 44. ${ }^{1} \mathrm{H}$ NMR (400 MHz, $\mathrm{CDCl}_{3}$ ) syn isomer: $\delta 5.84-5.72(\mathrm{~m}, 1 \mathrm{H}), 5.12-5.05(\mathrm{~m}, 2 \mathrm{H})$, $3.48-3.47(\mathrm{~m}, 1 \mathrm{H}), 2.31-2.16(\mathrm{~m}, 1 \mathrm{H}), 1.49(\mathrm{~m}, 2 \mathrm{H}), 1.27(\mathrm{~m}, 14 \mathrm{H}), 1.02(\mathrm{~d}, J=6.8 \mathrm{~Hz}$, $3 \mathrm{H}), 0.88(\mathrm{t}, J=6.8 \mathrm{~Hz}, 3 \mathrm{H})$; anti isomer: $3.38-3.39(\mathrm{~m}, 1 \mathrm{H}), 1.03(\mathrm{~d}, J=6.8 \mathrm{~Hz}, 3 \mathrm{H}) .{ }^{13} \mathrm{C}$ NMR (100 MHz, $\left.\mathrm{CDCl}_{3}\right)$ syn isomer: $\delta 140.4,115.0,74.7,43.4,34.0,31.9,29.7,29.6,29.6$, 29.3, 26.1, 22.6, 14.0, 14.0; anti isomer: $\delta 141.2,116.0,74.7,44.0,34.3,31.9,29.7,29.6$, $29.6,29.3,25.7,22.6,16.2,14.0$.

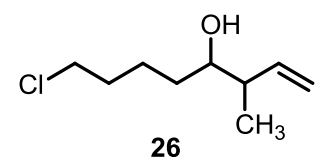

8-Chloro-3-methyloct-1-en-4-ol (26). Synthesized according to the general procedure with 5-chloropentanal $(0.2 \mathrm{mmol}, 24.0 \mathrm{mg})$ and 1,3 -butadiene $(0.4 \mathrm{mmol}, 0.2 \mathrm{ml}, 2 \mathrm{M}$ in THF solution) irradiated with blue LED for $16 \mathrm{~h}$. Purified by flash column chromatography on silica gel (PE : $\mathrm{DCM}=4: 1)$ to give $\mathbf{2 6}$ as a colourless oil $(28.2 \mathrm{mg}, 80 \%$ yield). syn isomer : anti isomer $=62: 38$. The relative configuration of $\mathbf{2 6}$ is determined according to the same method as 24. ${ }^{1} \mathrm{H}$ NMR $\left(400 \mathrm{MHz}, \mathrm{CDCl}_{3}\right)$ syn isomer: $\delta 5.83-5.70(\mathrm{~m}, 1 \mathrm{H}), 5.14-5.06(\mathrm{~m}$, $2 \mathrm{H}), 3.54(\mathrm{t}, J=6.8 \mathrm{~Hz}, 2 \mathrm{H}), 3.51-3.48(\mathrm{~m}, 1 \mathrm{H}), 2.32-2.16(\mathrm{~m}, 1 \mathrm{H}), 1.86-1.75(\mathrm{~m}, 2 \mathrm{H})$, $1.71-1.59(\mathrm{~m}, 1 \mathrm{H}), 1.56-1.35(\mathrm{~m}, 3 \mathrm{H}), 1.03(\mathrm{~d}, J=6.8 \mathrm{~Hz}, 3 \mathrm{H})$; anti isomer: $3.42-3.37$ $(\mathrm{m}, 1 \mathrm{H}), 1.04(\mathrm{~d}, J=6.8 \mathrm{~Hz}, 3 \mathrm{H}) .{ }^{13} \mathrm{C} \mathrm{NMR}\left(100 \mathrm{MHz}, \mathrm{CDCl}_{3}\right)$ syn isomer: $\delta 140.8,115.3$, 74.5, 44.9, 43.5, 33.2, 32.6, 23.5, 14.1; anti isomer: $\delta$ 140.1, 116.4, 74.4, 44.9, 44.1, 33.4, 32.6, 23.1, 16.2. HRMS (ESI) Calcd for $\mathrm{C}_{9} \mathrm{H}_{17} \mathrm{ClONa}[\mathrm{M}+\mathrm{Na}]^{+}$199.0866, Found 199.0859.

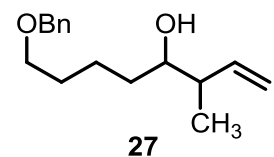

8-(Benzyloxy)-3-methyloct-1-en-4-ol (27). ${ }^{16}$ Synthesized according to the general procedure with 5-(benzyloxy)pentanal ( $0.2 \mathrm{mmol}, 38.4 \mathrm{mg}$ ) and 1,3-butadiene $(0.4 \mathrm{mmol}, 0.2 \mathrm{ml}, 2 \mathrm{M}$ in THF solution) irradiated with blue LED for $16 \mathrm{~h}$. Purified by flash column chromatography on silica gel ( $\mathrm{PE}: \mathrm{EA}=20: 1)$ to give 27 as a colourless oil $(42.1 \mathrm{mg}, 85 \%$ yield). syn isomer : anti isomer $=57: 43 .{ }^{1} \mathrm{H}$ NMR $\left(400 \mathrm{MHz}, \mathrm{CDCl}_{3}\right)$ syn isomer: $\delta 7.33(\mathrm{~d}, J=4.8 \mathrm{~Hz}$, 4H), $7.30-7.24(\mathrm{~m}, 1 \mathrm{H}), 5.82-5.70(\mathrm{~m}, 1 \mathrm{H}), 5.11-5.05(\mathrm{~m}, 2 \mathrm{H}), 4.50(\mathrm{~s}, 2 \mathrm{H}), 3.49-3.46$ (m, $3 \mathrm{H}), 2.32-2.15(\mathrm{~m}, 1 \mathrm{H}), 1.70-1.35(\mathrm{~m}, 6 \mathrm{H}), 1.01(\mathrm{~d}, J=6.8 \mathrm{~Hz}, 3 \mathrm{H})$; anti isomer: 3.40 $-3.37(\mathrm{~m}, 1 \mathrm{H}), 1.02(\mathrm{~d}, J=6.8 \mathrm{~Hz}, 3 \mathrm{H}) .{ }^{13} \mathrm{C}$ NMR $\left(100 \mathrm{MHz}, \mathrm{CDCl}_{3}\right)$ syn isomer: $\delta 141.0$, 138.6, 128.3, 127.6, 127.4, 115.1, 74.6, 72.9, 70.3, 43.4, 33.7, 29.7, 22.7, 14.1; anti isomer: $\delta$ 140.3, 138.6, 128.3, 127.6, 127.4, 116.1, 74.6, 72.9, 70.3, 44.0, 34.0, 29.7, 22.4, 16.2. HRMS (ESI) Calcd for $\mathrm{C}_{16} \mathrm{H}_{24} \mathrm{O}_{2} \mathrm{Na}[\mathrm{M}+\mathrm{Na}]^{+} 271.1674$, Found 271.1670.

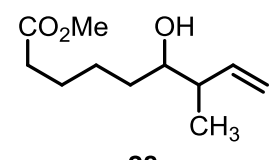


Methyl 6-hydroxy-7-methylnon-8-enoate (28). Synthesized according to the general procedure with methyl 6-oxohexanoate $(0.2 \mathrm{mmol}, 28.8 \mathrm{mg})$ and 1,3-butadiene $(0.4 \mathrm{mmol}$, $0.2 \mathrm{ml}, 2 \mathrm{M}$ in THF solution) irradiated with blue LED for $16 \mathrm{~h}$. Purified by flash column chromatography on silica gel (PE : EA $=20: 1)$ to give $\mathbf{2 8}$ as a colourless oil $(37.2 \mathrm{mg}, 93 \%$ yield). The relative configuration of $\mathbf{2 8}$ is determined according to the same method as $\mathbf{2 4}$. syn isomer : anti isomer $=64: 36 .{ }^{1} \mathrm{H} \mathrm{NMR}\left(400 \mathrm{MHz}, \mathrm{CDCl}_{3}\right)$ syn isomer: $\delta 5.83-5.71(\mathrm{~m}, 1 \mathrm{H})$, $5.12-5.06(\mathrm{~m}, 2 \mathrm{H}), 3.67(\mathrm{~s}, 3 \mathrm{H}), 3.48(\mathrm{~m}, 1 \mathrm{H}), 2.33(\mathrm{t}, J=7.6 \mathrm{~Hz}, 2 \mathrm{H}), 2.29-2.15(\mathrm{~m}, 1 \mathrm{H})$, $1.74-1.57(\mathrm{~m}, 2 \mathrm{H}), 1.53-1.47(\mathrm{~m}, 2 \mathrm{H}), 1.43-1.34(\mathrm{~m}, 2 \mathrm{H}), 1.02(\mathrm{~d}, J=6.8 \mathrm{~Hz}, 3 \mathrm{H})$; anti isomer: $3.67(\mathrm{~s}, 3 \mathrm{H}), 3.40(\mathrm{~m}, 1 \mathrm{H}), 1.03(\mathrm{~d}, J=6.8 \mathrm{~Hz}, 3 \mathrm{H}) .{ }^{13} \mathrm{C}$ NMR $\left(100 \mathrm{MHz}, \mathrm{CDCl}_{3}\right)$ syn isomer: $\delta 174.1,140.9,115.2,74.4,51.4,43.5,34.0,33.6,25.6,24.8,14.1$; anti isomer: $\delta$ 174.1, 140.2, 116.2, 74.4, 51.4, 44.1, 34.0, 33.8, 25.3, 24.9, 16.2. HRMS (ESI) Calcd for $\mathrm{C}_{11} \mathrm{H}_{20} \mathrm{O}_{3} \mathrm{Na}[\mathrm{M}+\mathrm{Na}]^{+}$223.1310, Found 223.1307.

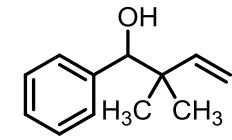

29<smiles>C=C(C)C(C)C(O)c1ccccc1</smiles>

30

2,2-Dimethyl-1-phenylbut-3-en-1-ol (29), ${ }^{17}$ syn-2,3-dimethyl-1-phenylbut-3-en-1-ol (30). ${ }^{18}$ Synthesized according to the general procedure with benzaldehyde $(0.2 \mathrm{mmol}, 21.2 \mathrm{mg})$ and isoprene $(0.4 \mathrm{mmol}, 27.2 \mathrm{mg})$ irradiated with blue LED for $16 \mathrm{~h}$. Purified by flash column chromatography on silica gel (PE : EA $=20: 1)$ to give a mixture of $\mathbf{2 9}$ and $\mathbf{3 0}$ (the ratio of $\mathbf{2 9}$ to 30 is $66: 34)$ as a colourless oil (20.1 mg, 57\% yield). 29: ${ }^{1} \mathrm{H}$ NMR (400 $\left.\mathrm{MHz}, \mathrm{CDCl}_{3}\right) \delta$ $7.25-7.22(\mathrm{~m}, 3 \mathrm{H}), 7.20-7.16(\mathrm{~m}, 2 \mathrm{H}), 5.83(\mathrm{dd}, J=17.2,10.8 \mathrm{~Hz}, 1 \mathrm{H}), 5.01$ (ddd, $J=12.0$, 10.8, $1.2 \mathrm{~Hz}, 2 \mathrm{H}$ ), 4.34 (d, $J=3.2 \mathrm{~Hz}, 1 \mathrm{H}$ ), 1.99 (brs, $1 \mathrm{H}), 0.93$ (s, 3H), 0.88 (s, 3H). ${ }^{13} \mathrm{C}$ NMR (101 MHz, $\left.\mathrm{CDCl}_{3}\right) \delta 145.1,140.8,127.8,127.5,127.4,113.7,80.7,42.2,24.4,21.1 .30$ : syn isomer : anti isomer $>20: 1 .{ }^{1} \mathrm{H}$ NMR $\left(400 \mathrm{MHz}, \mathrm{CDCl}_{3}\right) \delta 7.25-7.22(\mathrm{~m}, 3 \mathrm{H}), 7.20-$ $7.16(\mathrm{~m}, 2 \mathrm{H}), 4.78-4.76(\mathrm{~m}, 1 \mathrm{H}), 4.69(\mathrm{~m}, 1 \mathrm{H}), 4.64(\mathrm{dd}, J=5.2,2.8 \mathrm{~Hz}, 1 \mathrm{H}), 2.43-2.37$ $(\mathrm{m}, 1 \mathrm{H}), 1.88$ (brs, $1 \mathrm{H}), 1.65$ (s, 3H), $0.92(\mathrm{~d}, J=7.2 \mathrm{~Hz}, 3 \mathrm{H}) .{ }^{13} \mathrm{C} \mathrm{NMR}\left(100 \mathrm{MHz}, \mathrm{CDCl}_{3}\right) \delta$ 147.5, 143.1, 128.0, 127.1, 126.1, 111.9, 74.9, 48.0, 21.8, 13.0.

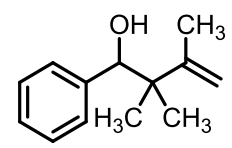

31

2,2,3-Trimethyl-1-phenylbut-3-en-1-ol (31). ${ }^{19}$ Synthesized according to the general procedure with benzaldehyde $(0.2 \mathrm{mmol}, 21.2 \mathrm{mg})$ and 2,3-dimethylbuta-1,3-diene ( $0.4 \mathrm{mmol}$, $32.8 \mathrm{mg}$ ) irradiated with blue LED for $16 \mathrm{~h}$. Purified by flash column chromatography on silica gel ( $\mathrm{PE}: \mathrm{EA}=20: 1)$ to give a mixture of $\mathbf{3 1}$ as a colourless oil $(25.1 \mathrm{mg}, 66 \%$ yield). ${ }^{1} \mathrm{H}$ NMR $\left(400 \mathrm{MHz}, \mathrm{CDCl}_{3}\right) \delta 7.35-7.24(\mathrm{~m}, 5 \mathrm{H}), 5.03(\mathrm{~s}, 1 \mathrm{H}), 4.96(\mathrm{~s}, 1 \mathrm{H}), 4.63(\mathrm{~s}, 1 \mathrm{H})$, 2.09 (brs, 1H), 1.86 (s, 3H), 0.99 (s, 3H), 0.94 (s, 3H). ${ }^{13} \mathrm{C} \mathrm{NMR}\left(100 \mathrm{MHz}, \mathrm{CDCl}_{3}\right) \delta$ 150.7, 140.4, 128.0, 127.4, 127.3, 113.1, 77.6, 44.5, 24.2, 20.2, 19.8. 


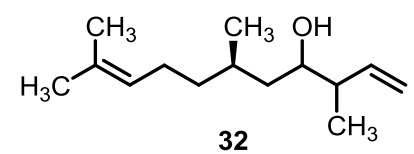

(6R)-3,6,10-Trimethylundeca-1,9-dien-4-ol (32). ${ }^{20}$ Synthesized according to the general procedure with $(R)$-3,7-dimethyloct-6-enal $(0.2 \mathrm{mmol}, 30.8 \mathrm{mg})$ and 1,3-butadiene $(0.4 \mathrm{mmol}$, $0.2 \mathrm{ml}, 2 \mathrm{M}$ in THF solution) irradiated with blue LED for $16 \mathrm{~h}$. Purified by flash column chromatography on silica gel ( $\mathrm{PE}: \mathrm{EA}=20: 1$ ) to give a mixture of $\mathbf{3 2}$ (mixture of four diastereoisomers) as a colourless oil $\left(36.9 \mathrm{mg}, 88 \%\right.$ yield). ${ }^{1} \mathrm{H}$ NMR (400 $\left.\mathrm{MHz}, \mathrm{CDCl}_{3}\right) \delta$ $5.85-5.71(\mathrm{~m}, 1 \mathrm{H}), 5.13-5.06(\mathrm{~m}, 3 \mathrm{H}), 3.64-3.47(\mathrm{~m}, 1 \mathrm{H}), 2.27-2.14(\mathrm{~m}, 1 \mathrm{H}), 2.07-$ $1.88(\mathrm{~m}, 2 \mathrm{H}), 1.68(\mathrm{~s}, 3 \mathrm{H}), 1.60(\mathrm{~s}, 3 \mathrm{H}), 1.48-1.15(\mathrm{~m}, 5 \mathrm{H}), 1.05-0.89(\mathrm{~m}, 3 \mathrm{H}) .{ }^{13} \mathrm{C} \mathrm{NMR}$ $\left(100 \mathrm{MHz}, \mathrm{CDCl}_{3}\right) \delta 141.2,141.0,140.4,140.1,131.1,124.8,116.2,115.2,72.8,72.6$ 72.3, 44.9, 44.2, 44.1, 43.5, 42.1, 41.7, 41.5, 41.3, 38.2, 38.1, 36.3, 36.3, 29.4, 29.3, 29.0, 29.0, 25.7, $25.5,25.5,25.3,20.5,19.0,19.0,17.6,16.3,16.2,14.4,13.7$. HRMS (ESI) Calcd for $\mathrm{C}_{14} \mathrm{H}_{26} \mathrm{ONa}[\mathrm{M}+\mathrm{Na}]^{+}$233.1881, Found 233.1875.

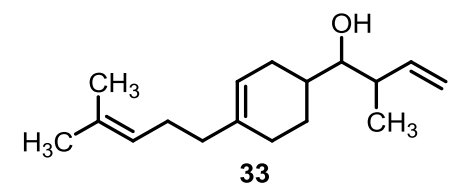

2-methyl-1-(4-(4-methylpent-3-en-1-yl)cyclohex-3-en-1-yl)but-3-en-1-ol (33). Synthesized according to the general procedure with $(0.2 \mathrm{mmol}, 38.4 \mathrm{mg})$ and 1,3 -butadiene $(0.4 \mathrm{mmol}$, $0.2 \mathrm{ml}, 2 \mathrm{M}$ in THF solution) irradiated with blue LED for $16 \mathrm{~h}$. Purified by flash columnchromatography on silica gel (PE : EA $=20: 1)$ to give a mixture of $\mathbf{3 3}$ (mixture of diastereomeric and conformational isomers) as a colorless oil (26.3 mg, 53\% yield). ${ }^{1} \mathrm{H}$ NMR $\left(400 \mathrm{MHz}, \mathrm{CDCl}_{3}\right) \delta 5.90-5.74(\mathrm{~m}, 1 \mathrm{H}), 5.40-5.38(\mathrm{~m}, 1 \mathrm{H}), 5.14-5.06(\mathrm{~m}, 3 \mathrm{H}), 3.35-$ $3.17(\mathrm{~m}, 1 \mathrm{H}), 2.48-2.32(\mathrm{~m}, 1 \mathrm{H}), 2.08-1.89(\mathrm{~m}, 8 \mathrm{H}), 1.68(\mathrm{~s}, 3 \mathrm{H}), 1.68-1.60(\mathrm{~m}, 1 \mathrm{H})$, $1.60(\mathrm{~s}, 3 \mathrm{H}), 1.52-1.21(\mathrm{~m}, 2 \mathrm{H}), 1.08-1.01(\mathrm{~m}, 3 \mathrm{H}) .{ }^{13} \mathrm{C} \mathrm{NMR}\left(100 \mathrm{MHz}, \mathrm{CDCl}_{3}\right) \delta 142.1$, 142.0, 141.7, 140.5, 140.5, 139.8, 139.54, 138.2, 137.9, 137.5, 137.3, 137.2, 137.2, 137.0, $136.7,131.4,131.3,124.4,124.4,124.3,121.00,120.8,120.4,120.2,120.1,120.1,119.8$, 119.5, 116.3, 116.2, 116.2, 114.8, 114.8, 114.8, 78.4, 78.3, 78.2, 78.2, 78.1, 77.8, 77.7, 41.2, $41.1,40.4,40.4,40.3,39.6,39.5,38.0,38.0,37.9,37.9,37.6,37.3,37.1,36.9,36.7,36.6,36.5$, $36.3,36.3,31.9,31.7,29.6,28.9,28.6,28.4,28.4,28.3,26.6,26.5,26.5,26.5,26.3,26.3$, 26.2, 25.8, 25.7, 25.6, 25.4, 25.3, 25.2, 24.9, 24.3, 24.2, 23.6, 17.7, 17.7, 17.2, 17.2, 16.9, 16.9, 13.7, 13.6, 12.4, 12.1. HRMS (ESI) Calcd for $\mathrm{C}_{17} \mathrm{H}_{28} \mathrm{ONa}[\mathrm{M}+\mathrm{Na}]^{+}$271.2038, Found 271.2031 .

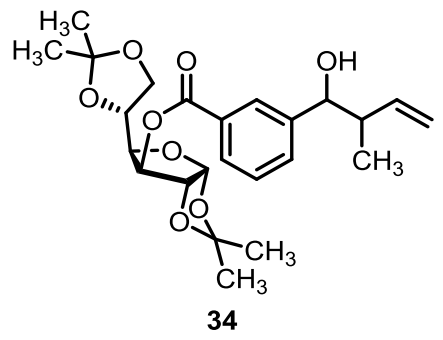




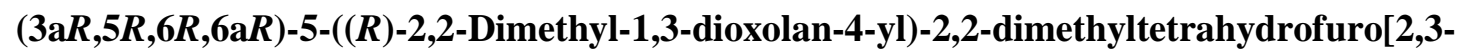
$d][1,3]$ dioxol-6-yl3-(1-hydroxy-2-methylbut-3-en-1-yl)benzoate (34). Synthesized according to the general procedure with $(3 \mathrm{a} R, 5 R, 6 R, 6 \mathrm{a} R)-5-((R)-2,2$-dimethyl-1,3-dioxolan-4yl)-2,2-dimethyltetrahydrofuro[2,3- $d][1,3]$ dioxol-6-yl 3-formylbenzoate $(0.2 \mathrm{mmol}, 78.4 \mathrm{mg})$ and 1,3-butadiene ( $0.4 \mathrm{mmol}, 0.2 \mathrm{ml}, 2 \mathrm{M}$ in THF solution) irradiated with blue LED for $20 \mathrm{~h}$. Purified by flash column chromatography on silica gel (PE : EA $=20: 1)$ to give a mixture of $\mathbf{3 4}$ as a pale yellow oil $(57.3 \mathrm{mg}, 64 \%$ yield). relative configuration of $\mathbf{3 4}$ is determined according to the same method as $\mathbf{6}$ and 10. syn isomer : anti isomer $=59: 41 .{ }^{1} \mathrm{H}$ NMR (400 $\left.\mathrm{MHz}, \mathrm{CDCl}_{3}\right)$ syn isomer: $\delta 7.99-7.90(\mathrm{~m}, 2 \mathrm{H}), 7.60-7.54(\mathrm{~m}, 1 \mathrm{H}), 7.46-7.41(\mathrm{~m}, 1 \mathrm{H})$, $5.96-5.95(\mathrm{~m}, 1 \mathrm{H}), 5.83-5.71(\mathrm{~m}, 1 \mathrm{H}), 5.50(\mathrm{~m}, 1 \mathrm{H}), 5.22-5.18(\mathrm{~m}, 1 \mathrm{H}), 5.10-5.03(\mathrm{~m}$, $1 \mathrm{H}), 4.69(\mathrm{t}, J=4.4 \mathrm{~Hz}, 1 \mathrm{H}), 4.64(\mathrm{~d}, J=3.6 \mathrm{~Hz}, 1 \mathrm{H}), 4.39-4.32(\mathrm{~m}, 2 \mathrm{H}), 4.14-4.06(\mathrm{~m}$, $2 \mathrm{H}), 2.63-2.55(\mathrm{~m}, 1 \mathrm{H}), 2.14$ (brs, $1 \mathrm{H}), 1.56(\mathrm{~s}, 3 \mathrm{H}), 1.42(\mathrm{~s}, 3 \mathrm{H}), 1.32(\mathrm{~s}, 3 \mathrm{H}), 1.27(\mathrm{~s}, 3 \mathrm{H})$, $0.99(\mathrm{dd}, J=6.8,3.2 \mathrm{~Hz}, 3 \mathrm{H})$; anti isomer: $4.45(\mathrm{~d}, J=6.0, \mathrm{~Hz}, 1 \mathrm{H}), 2.53-2.44(\mathrm{~m}, 1 \mathrm{H})$, 2.31 (brs, $1 \mathrm{H}), 0.90(\mathrm{dd}, J=6.8,3.2 \mathrm{~Hz}, 3 \mathrm{H}) .{ }^{13} \mathrm{C} \mathrm{NMR}\left(100 \mathrm{MHz}, \mathrm{CDCl}_{3}\right) \delta 165.2,165.2$, $143.3,143.2$, 139.9, 139.8, 132.0, 131.9, 131.7, 131.5, 129.5, 129.5, 129.5, 129.4, 129.3, $128.9,128.9,128.6,128.6,128.5,128.4,128.3,128.3,128.2,128.1,127.8,127.7,117.4$, 116.1, 116.0, 112.3, 109.4, 105.1, 83.4, 80.0, 77.2, 76.6, 76.5, 72.6, 67.2, 46.3, 44.6, 44.6, 26.8, 26.7, 26.2, 25.2, 16.4, 13.8, 13.7. HRMS (ESI) Calcd for $\mathrm{C}_{24} \mathrm{H}_{32} \mathrm{O}_{8} \mathrm{Na}[\mathrm{M}+\mathrm{Na}]^{+}$ 471.1995, Found 471.1990. 


\section{Examples of Less Successful Substrates}

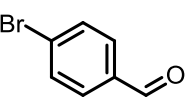

$0 \%$ Yield

Reduction of the bromide<smiles>Cn1cc(C=O)c2ccccc21</smiles>

$0 \%$ Yield complicated reaction<smiles>C=CC(=C)CCC=C(C)C</smiles>

$0 \%$ Yield complicated reaction

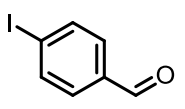

$0 \%$ Yield Reduction of the lodide<smiles>O=Cc1cncnc1</smiles>

$0 \%$ Yield complicated reaction<smiles>C=C/C=C/CC</smiles>

$<5 \%$ Yield complicated reaction<smiles>N#Cc1ccc(C=O)cc1</smiles>

0\% Yield Starting Material Remaining<smiles>O=C/C=C/c1ccccc1</smiles>

$0 \%$ Yield complicated reaction<smiles>C=CC(=C)c1ccccc1</smiles>

$<5 \%$ Yield complicated reaction<smiles>O=Cc1cccc2ccccc12</smiles>

$0 \%$ Yield complicated reaction
$0 \%$ Yield Strating Material Remaining<smiles>C1=CCCC=C1</smiles>

$0 \%$ Yield complicated reaction 


\section{Control Experiments and Mechanistic Studies}

\subsection{Control Experiments}

In order to confirm the effect of each catalyst component, control experiments were conducted (Table S1). As a result, all the Ni salt, ligand, photocatalyst, proton source, and photoirradiation were proved to be indispensable for this transformation (entries 1-6), supporting the validity of our reaction design described in Scheme 1b. In addition, when 5 equiv ${ }^{i} \mathrm{Pr}_{2} \mathrm{NEt}$ was employed as a reductant instead of $\mathrm{HE}, 3$ was obtained in $40 \%$ yield. This result indicates that ${ }^{i} \mathrm{Pr}_{2} \mathrm{NEt}$ can function as both a reductant and a proton source albeit with low efficiency comparing with HE in this transformation.
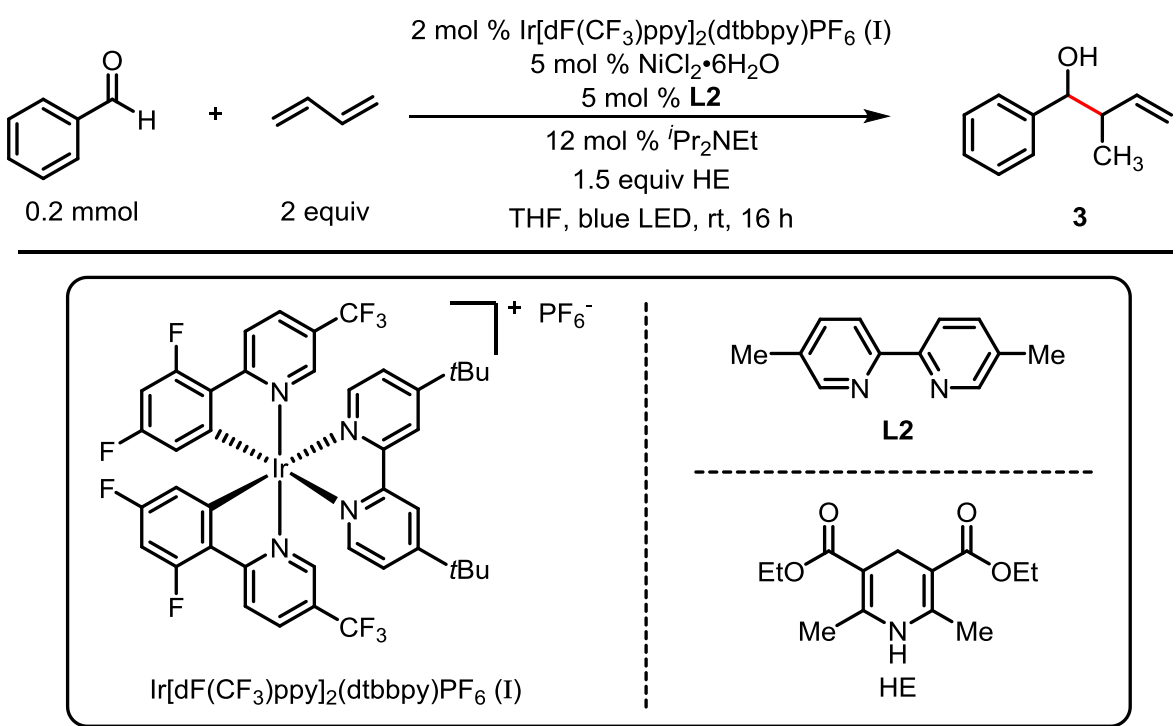

\begin{tabular}{|c|c|c|c|}
\hline Entry $^{a}$ & Change from "standard conditions" & Yield $(\%)^{\mathrm{b}}$ & $\mathrm{dr}^{b}$ \\
\hline 1 & none & 86 & $1.9: 1$ \\
\hline 2 & without I & 0 & - \\
\hline 3 & without $\mathrm{NiCl}_{2} \cdot 6 \mathrm{H}_{2} \mathrm{O}$ & 0 & - \\
\hline 4 & without $\mathbf{L 2}$ & 0 & - \\
\hline 5 & without ${ }^{i} \mathrm{Pr}_{2} \mathrm{NEt}$ & $<5$ & - \\
\hline 6 & Without blue LED irradiation & 0 & - \\
\hline 7 & 5 equiv ${ }^{i} \mathrm{Pr}_{2} \mathrm{NEt}$ instead of $12 \mathrm{~mol} \%{ }^{i} \mathrm{Pr}_{2} \mathrm{NEt}$ and $\mathrm{HE}$ & 40 & 1.9:1 \\
\hline
\end{tabular}

${ }^{a}$ All reaction were performed with $2 \mathrm{~mol} \% \operatorname{Ir}\left[\mathrm{dF}_{\left(\mathrm{CF}_{3}\right) \mathrm{ppy}}\right]_{2}(\mathrm{dtbbpy}) \mathrm{PF}_{6}, 5 \mathrm{~mol} \% \mathrm{NiCl}_{2} \bullet 6 \mathrm{H}_{2} \mathrm{O}, 5 \mathrm{~mol} \% \mathbf{L 2}, 12 \mathrm{~mol} \%{ }^{i} \mathrm{Pr}_{2} \mathrm{NEt}$, and 1.5 equiv $\mathrm{HE}$ with $5 \mathrm{~W}$ blue LED if otherwise noted. ${ }^{b}$ Determined by crude ${ }^{1} \mathrm{H}$ NMR with 1,1,2,2-tetrachloroethane as an internal standard.

\section{Synthesis of $\left[\mathrm{L} 2 \mathrm{NiCl}_{2}\right]$ complex:}

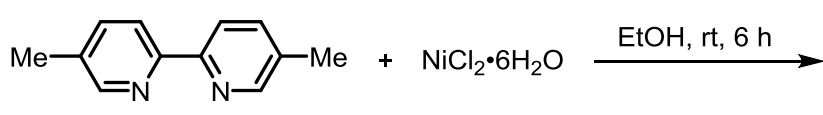

L2

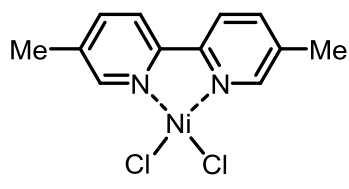

$\mathbf{L} 2 \mathrm{NiCl}_{2}, 90 \%$

A solution of $\mathbf{L 2}(117 \mathrm{mg}, 0.64 \mathrm{mmol})$ in $\mathrm{EtOH}(2 \mathrm{~mL})$ was added to a solution of $\mathrm{NiCl}_{2} \bullet 6 \mathrm{H}_{2} \mathrm{O}(152 \mathrm{mg}, 0.64 \mathrm{mmol})$ in EtOH $(2 \mathrm{~mL})$. After stirring at room temperature for $6 \mathrm{~h}$, 
a pale green precipitate was formed and filtered off, washed with EtOH $(3 \times 3 \mathrm{~mL})$, and dried in vacuo to afford complex [ $\left.\mathbf{L} 2 \mathrm{NiCl}_{2}\right]$ as a pale green solid $(179.7 \mathrm{mg}, 90 \%$ yield). HRMS (ESI) Calcd for $\mathrm{C}_{12} \mathrm{H}_{12} \mathrm{Cl}_{2} \mathrm{~N}_{2} \mathrm{NiNa}[\mathrm{M}+\mathrm{Na}]^{+} 334.9629$, Found 334.9615 .

\section{Photoredox Ni-catalyzed Branch-Selective Allylation of Aldehydes with [ $\left.\mathbf{L 2 N i C l}_{2}\right]$ :}
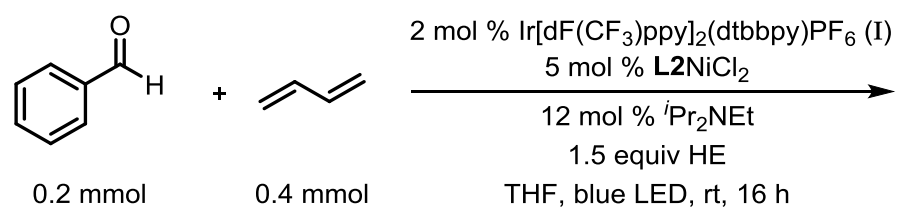

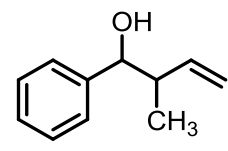

$3,88 \%, 1.9: 1 \mathrm{dr}$

In an argon-filled glovebox, a $25 \mathrm{~mL}$ Schlenk tube was charged with benzaldehyde $(0.2 \mathrm{mmol}$, $21.2 \mathrm{mg}$ ), 1,3-butadiene ( $0.4 \mathrm{mmol}, 0.2 \mathrm{ml}, 2 \mathrm{M}$ in THF solution), Hantzsch ester (HE) (0.3 mmol, $75.9 \mathrm{mg}), \operatorname{Ir}\left[\mathrm{dF}\left(\mathrm{CF}_{3}\right) \text { ppy }\right]_{2}(\mathrm{dtbbpy}) \mathrm{PF}_{6}$ (I) $(0.004 \mathrm{mmol}, 4.5 \mathrm{mg}), \mathbf{L} 2 \mathrm{NiCl}_{2}(0.001$ mmol, $3.12 \mathrm{mg}),{ }^{i} \mathrm{Pr}_{2} \mathrm{NEt}(0.024 \mathrm{mmol}, 3.10 \mathrm{mg})$, and dry THF $(2 \mathrm{~mL})$. The tube was removed from the glove box, and irradiated with blue LED $(5 \mathrm{~W})$. The reaction mixture was stirred at room temperature for $16 \mathrm{~h}$. After filtration and concentration, the resulting mixture was determined by crude ${ }^{1} \mathrm{H}$ NMR with 1,1,2,2-tetrachloroethane $(0.2 \mathrm{mmol}, 33.6 \mathrm{mg})$ as an internal standard to give a yield of $88 \%$.

\subsection{Radical Trapping Experiments}<smiles>CC(=O)c1ccccc1</smiles>

$0.2 \mathrm{mmol}$

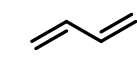

$0.6 \mathrm{mmol}$

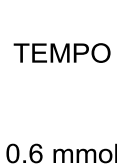

$2 \mathrm{~mol} \% \operatorname{Ir}\left[\mathrm{dF}\left(\mathrm{CF}_{3}\right) \mathrm{ppy}\right]_{2}(\mathrm{dtbbpy}) \mathrm{PF}_{6}(\mathrm{I})$ $10 \mathrm{~mol} \% \mathrm{NiCl}_{2} \cdot 6 \mathrm{H}_{2} \mathrm{O}$

$10 \mathrm{~mol} \% \mathrm{~L} 2$

1.5 equiv $\mathrm{HE}$

THF, blue LED, rt, $16 \mathrm{~h}$

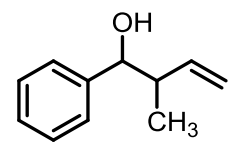

$71 \%$

In an argon-filled glovebox, a $25 \mathrm{~mL}$ Schlenk tube was charged with benzaldehyde $(0.2 \mathrm{mmol}$, $21.2 \mathrm{mg}$ ), 1,3-butadiene ( $0.6 \mathrm{mmol}, 0.3 \mathrm{ml}, 2 \mathrm{M}$ in THF solution), TEMPO (0.6 mmol, 93.7 $\mathrm{mg})$, Hantzsch ester (HE) $(0.3 \mathrm{mmol}, 75.9 \mathrm{mg}), \operatorname{Ir}\left[\mathrm{dF}\left(\mathrm{CF}_{3}\right) \mathrm{ppy}\right]_{2}(\mathrm{dtbbpy}) \mathrm{PF}_{6}(\mathbf{I})(0.004 \mathrm{mmol}$, $4.5 \mathrm{mg}), \mathrm{NiCl}_{2} \cdot 6 \mathrm{H}_{2} \mathrm{O}(0.02 \mathrm{mmol}, 4.8 \mathrm{mg}), \mathbf{L 2}(0.02 \mathrm{mmol}, 4.0 \mathrm{mg}),{ }^{i} \operatorname{Pr}_{2} \mathrm{NEt}(0.024 \mathrm{mmol}$, $3.1 \mathrm{mg})$, and dry THF $(2 \mathrm{~mL})$. The tube was removed from the glove box, and irradiated with blue LED $(5 \mathrm{~W})$ at room temperature for $16 \mathrm{~h}$. After filtration and concentration, the resulting mixture was determined by crude ${ }^{1} \mathrm{H}$ NMR with 1,1,2,2-tetrachloroethane $(0.2 \mathrm{mmol}, 33.6 \mathrm{mg})$ as an internal standard to give a yield of $71 \%$.<smiles>CC(=O)c1ccccc1</smiles>

$0.2 \mathrm{mmol}$

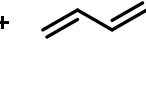

$0.6 \mathrm{mmol}$<smiles>C=C(c1ccccc1)c1ccccc1</smiles>

$0.6 \mathrm{mmol}$
$2 \mathrm{~mol} \% \operatorname{Ir}\left[\mathrm{dF}\left(\mathrm{CF}_{3}\right) \mathrm{ppy}\right]_{2}(\mathrm{dtbbpy}) \mathrm{PF}_{6}(\mathrm{I})$

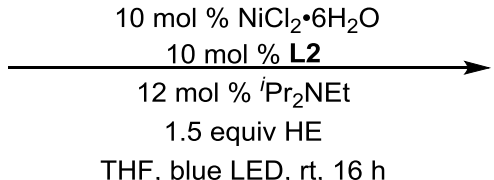<smiles>C=CC(C)C(O)c1ccccc1</smiles>

In an argon-filled glovebox, a $25 \mathrm{~mL}$ Schlenk tube was charged with benzaldehyde $(0.2 \mathrm{mmol}$, $21.2 \mathrm{mg}$ ), 1,3-butadiene ( $0.6 \mathrm{mmol}, 0.3 \mathrm{ml}, 2 \mathrm{M}$ in THF solution), ethene-1,1-diyldibenzene (0.6 mmol, $108.1 \mathrm{mg})$, Hantzsch ester (HE) (0.3 mmol, $75.9 \mathrm{mg}), \operatorname{Ir}\left[\mathrm{dF}\left(\mathrm{CF}_{3}\right) \mathrm{ppy}\right]_{2}(\mathrm{dtbbpy}) \mathrm{PF}_{6}$ 
(I) $(0.004 \mathrm{mmol}, 4.5 \mathrm{mg}), \mathrm{NiCl}_{2} \cdot 6 \mathrm{H}_{2} \mathrm{O}(0.02 \mathrm{mmol}, 4.8 \mathrm{mg}), 5,5$ '-dimethyl-2,2'-bipyridine $(0.02 \mathrm{mmol}, 4.0 \mathrm{mg}),{ }^{i} \mathrm{Pr}_{2} \mathrm{NEt}(0.024 \mathrm{mmol}, 3.1 \mathrm{mg})$ and dry THF $(2 \mathrm{~mL})$. The tube was removed from the glove box, and irradiated with blue LED $(5 \mathrm{~W})$ at room temperature for 16 h. After filtration and concentration, the resulting mixture was determined by crude ${ }^{1} \mathrm{H}$ NMR with 1,1,2,2-tetrachloroethane $(0.2 \mathrm{mmol}, 33.6 \mathrm{mg})$ as an internal standard to give a yield of $72 \%$.

\subsection{Radical Clock Experiment}
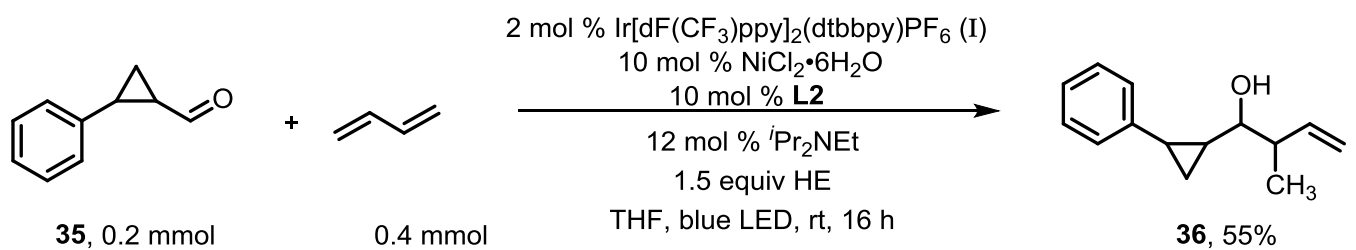

In an argon-filled glovebox, a $25 \mathrm{~mL}$ Schlenk tube was charged with 2-phenylcyclopropane-1-carbaldehyde 35 ( $0.2 \mathrm{mmol}, 29.2 \mathrm{mg})$, 1,3-butadiene ( $0.4 \mathrm{mmol}, 0.2$ $\mathrm{ml}, \quad 2 \mathrm{M}$ in THF solution), Hantzsch ester (HE) (0.3 mmol, $75.9 \mathrm{mg})$,

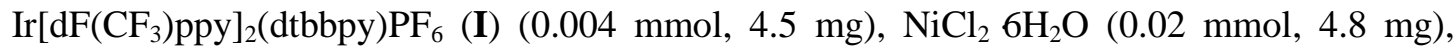
5,5'-dimethyl-2,2'-bipyridine $\mathbf{L} 2(0.02 \mathrm{mmol}, 4.0 \mathrm{mg}),{ }^{i} \operatorname{Pr}_{2} \mathrm{NEt}(0.024 \mathrm{mmol}, 3.1 \mathrm{mg})$ and dry THF ( $2 \mathrm{~mL}$ ). The tube was removed from the glove box, and irradiated with blue LED ( $5 \mathrm{~W}$ ) at room temperature for $16 \mathrm{~h}$. The mixture was then concentrated in vacuo. And the crude product was purified by flash column chromatography (silica gel, PE : EA $=10: 1$ ) to afford the desired homoallylic alcohol 36 (mixture of stereoisomers) as pale yellow oil (22.2 $\mathrm{mg}, 55 \%$ yield). ${ }^{1} \mathrm{H}$ NMR (400 MHz, $\left.\mathrm{CDCl}_{3}\right): \delta 7.27-7.23(\mathrm{~m}, 2 \mathrm{H}), 7.17-7.12(\mathrm{~m}, 1 \mathrm{H}), 7.10-7.04(\mathrm{~m}$, $2 \mathrm{H}), 5.95-5.78(\mathrm{~m}, 1 \mathrm{H}), 5.17-5.05(\mathrm{~m}, 2 \mathrm{H}), 3.14-2.95(\mathrm{~m}, 1 \mathrm{H}), 2.52-2.35(\mathrm{~m}, 1 \mathrm{H}), 1.96-$ $1.80(\mathrm{~m}, 1 \mathrm{H}), 1.26(\mathrm{~m}, 1 \mathrm{H}), 1.13-1.08(\mathrm{~d}, J=6.8 \mathrm{~Hz}, 3 \mathrm{H}), 1.05-0.93(\mathrm{~m}, 2 \mathrm{H}){ }^{13} \mathrm{C}$ NMR $(100$ $\left.\mathrm{MHz}, \mathrm{CDCl}_{3}\right) \delta 142.7,142.7,142.3,140.7,140.6,140.5,140.3,128.3,128.3,125.9,125.9$, $125.8,125.8,125.6,125.6,125.5,116.2,116.0,115.4,115.3,78.5,78.4,78.3,78.2,45.2,44.8$ 44.0, 43.9, 27.3, 27.2, 26.9, 26.7, 21.7, 21.6, 20.5, 20.4, 16.3, 14.9, 14.7, 14.0, 13.1, 12.9. HRMS (ESI) Calcd. for $\mathrm{C}_{14} \mathrm{H}_{18} \mathrm{ONa}[\mathrm{M}+\mathrm{Na}]^{+} 225.1255$, Found 225.1245 . 


\section{References}

1. (a) Sakata, Y.; Yasui, E.; Takatori, K.; Suzuki, Y.; Mizukami, M.; Nagumo, S. J. Org. Chem. 2018, 83, 9103. (b) Spallarossa, M.; Wang, Q.; Riva, R.; Zhu, J. Org. Lett. 2016, 18, 1622. (c) Sabbatani, J., Maulide, N. Angew. Chem. Int. Ed. 2016, 55, 6780.

2. Lee, K. N.; Lei, Z.; Ngai, M. Y. J. Am. Chem. Soc. 2017, 139, 5003.

3. Lowry, M. S.; Bernhard, S. Chem. Mater. 2005, 17, 5712.

4. Singh, K.; Banerjee, D. J. Org. Chem. 2018, 83, 15406.

5. Kobayashi, S.; Nishio, K. J. Org. Chem. 1994, 59, 6620.

6. Shibata, I.; Yoshimura, N.; Yabu, M.; Baba, A. Eur. J. Org. Chem. 2001, 3207.

7. Jing, S.; Agosston, E. G.; Chen, T.; Cabel, M. P.; Turos, E. Organometallics 1995, 14, 4697.

8. Fujita, K.; Oshima, K. J. Am. Chem. Soc. 2004, 126, 6776.

9. Sai, M.; Yorimitsu, H.; Oshima, K. Angew. Chem. Int. Ed. 2011, 50, 3294.

10. Yoo, J.; Oh, K. E.; Keum, G.; Kang, S. B.; Kim, Y. Polyhedron. 2000, 19, 549.

11. (a) Takahara, J.; Masuyama, Y.; Kurusu, Y. J. Am. Chem. Soc. 1992, 114, 2577. (b) Weber, F.; Ballmann, M.; Kohlmeyer, C.; Hilt, G. Org. Lett. 2016, 18, 548.

12. Tian, Y. F.; Chen, Y. Tetrahedron Lett. 2018, 59, 3206.

13. Denmark, S. E.; Fu, J. J. Am. Chem. Soc. 2001, 123, 9488.

14. McCluskey, A.; Muderawan, I. W.; Young, D. J. J. Org. Chem. 2001, 66, 7811.

15. Millán, A.; Campana, A. G.; Bazdi, B.; Miguel, D.; Alvarez de Cienfuegos, L.; Echavarren, A. M.; Cuerva, J. M. Chem. Eur. J. 2011, 17, 3985.

16. Smith III, A. B.; Lin, Q.; Doughty, V. A.; Zhuang, L.; McBriar, M. D.; Kerns, J. K.; Bennett, C. S. Tetrahedron, 2009, 65, 6470.

17. Sumida, S. I.; Ohga, M.; Mitani, J.; Nokami, J. J. Am. Chem. Soc. 2000, 122, 1310.

18. Ishihara, K.; Mouri, M.; Gao, Q.; Maruyama, T.; Furuta, K.; Yamamoto, H. J. Am. Chem. Soc. 1993, 115, 11490.

19. (a) Suzuki, I.; Uji, Y.; Kanaya, S.; Ieki, R.; Tsunoi, S.; Shibata, I. Org. Lett. 2017, 19, 5392. (b) Kimura, M.; Nojiri, D.; Fukushima, M.; Oi, S.; Sonoda, Y.; Inoue, Y. Org. Lett. 2009, 11, 3794.

20. Szymoniak, J.; Thery, N.; Moïse, C. Bull. Soc. Chim. Fr. 1997, 1, 85. 


\section{NMR Spectra}

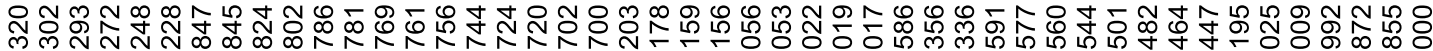

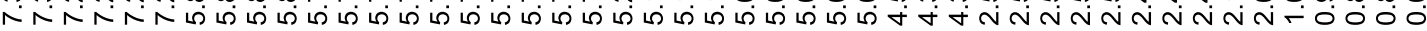<smiles>C=CC(C)C(O)c1ccccc1</smiles>
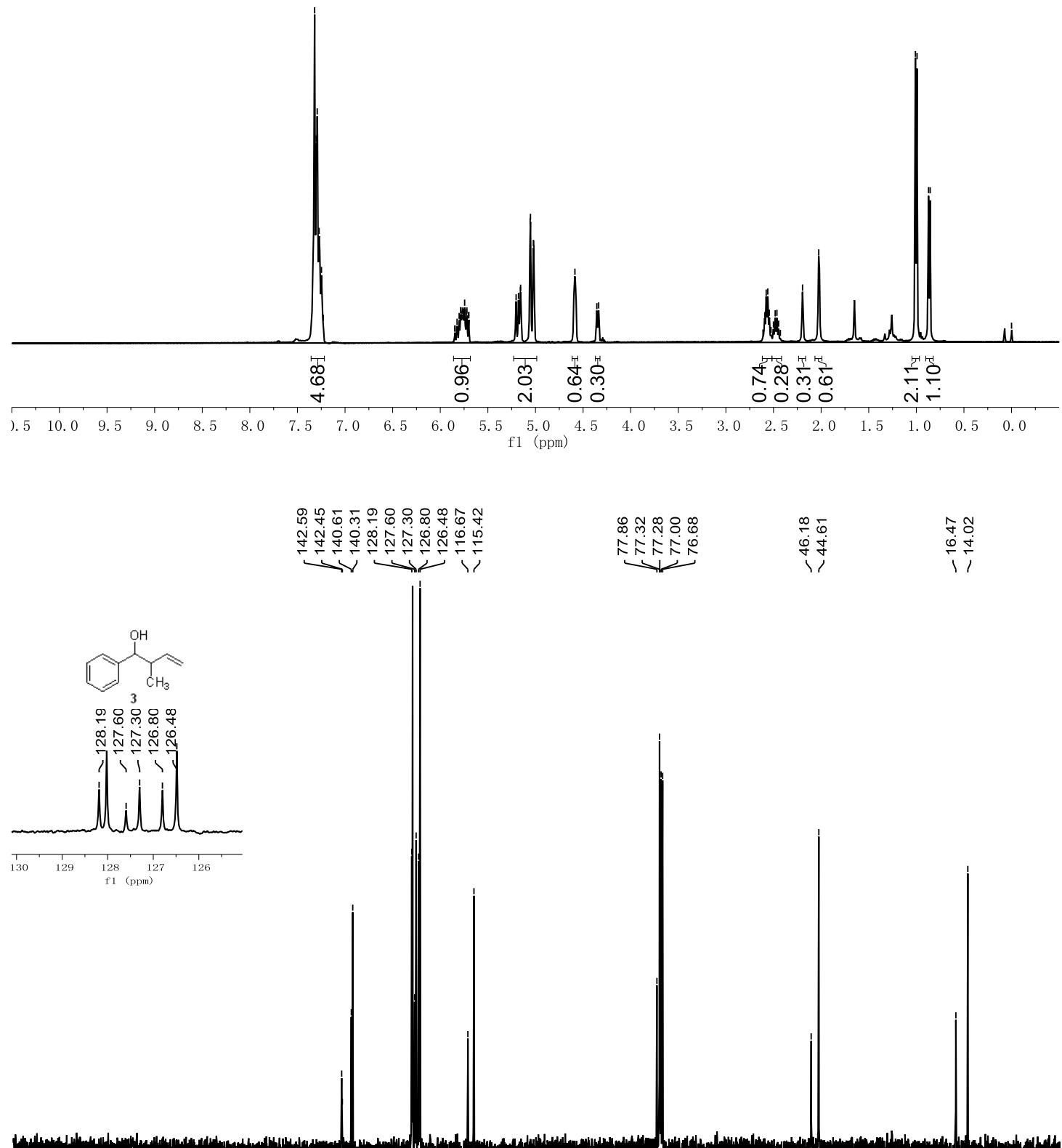


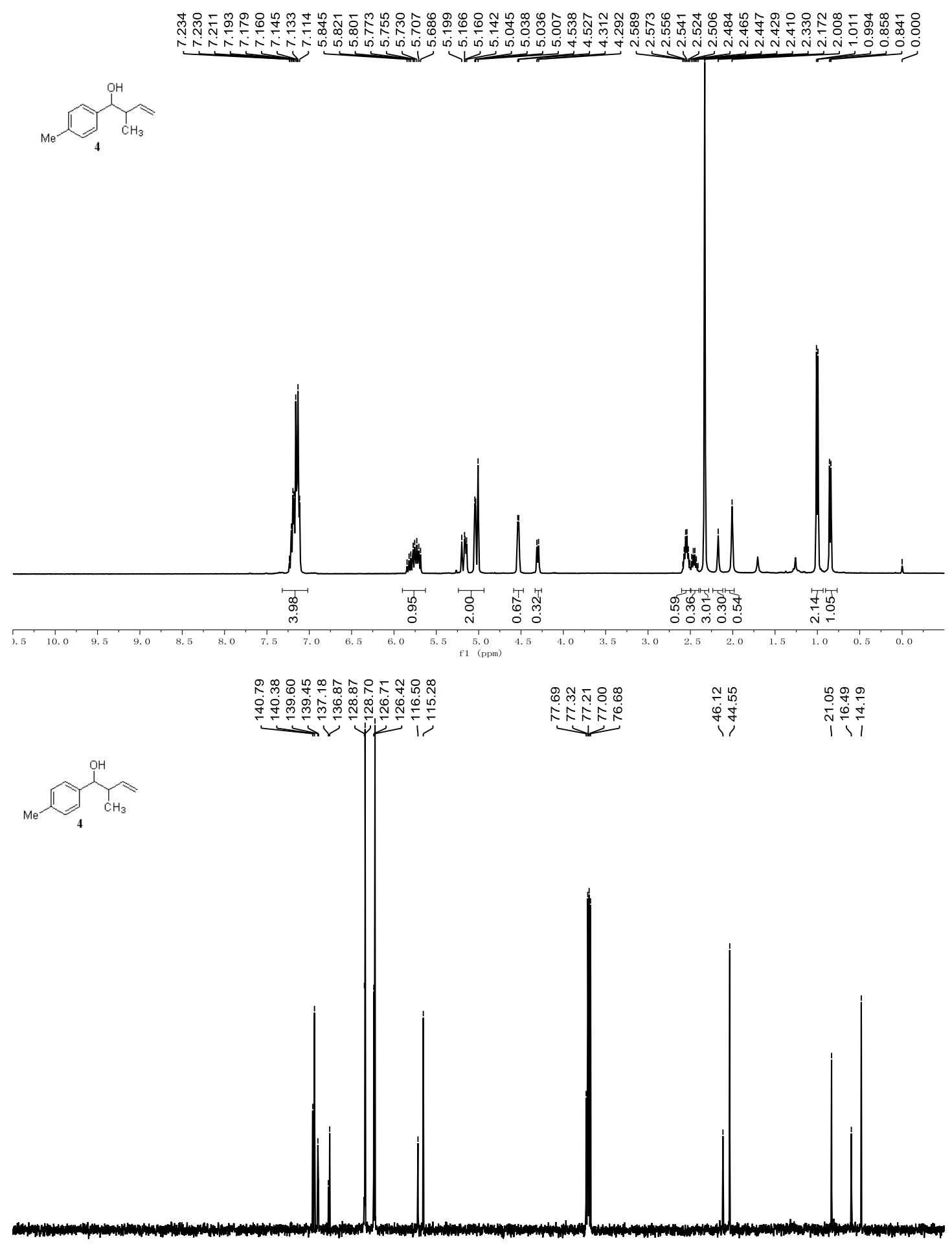

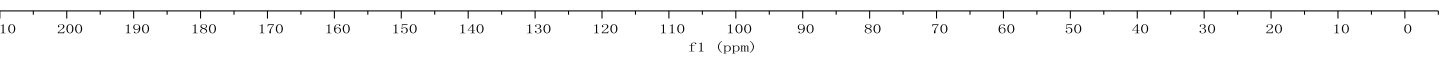




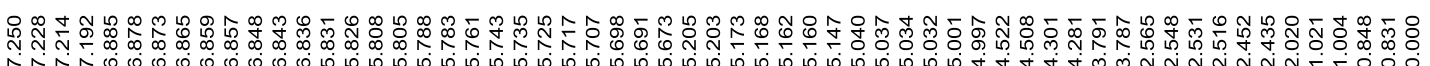
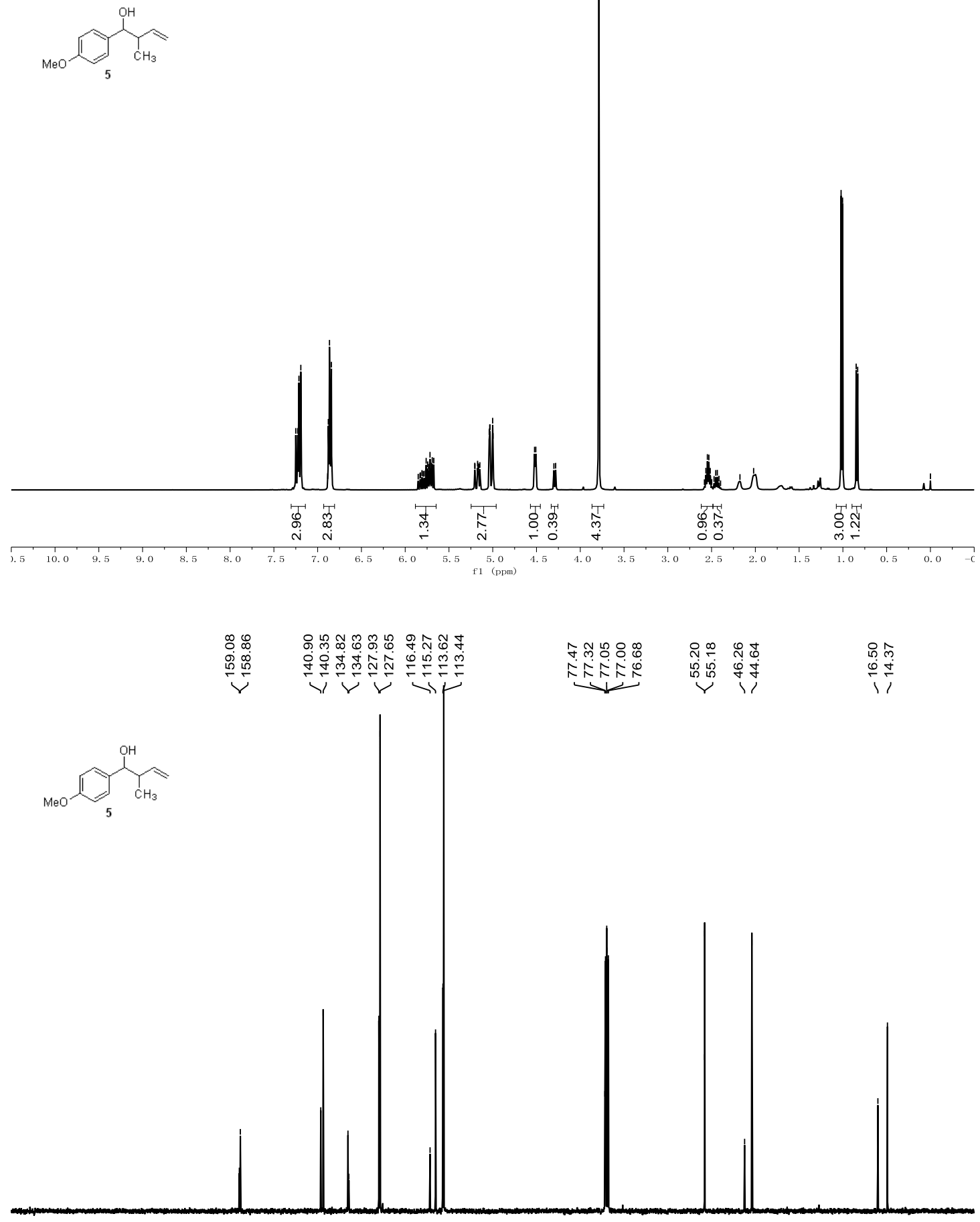

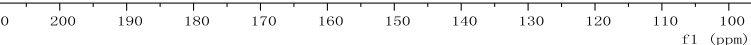




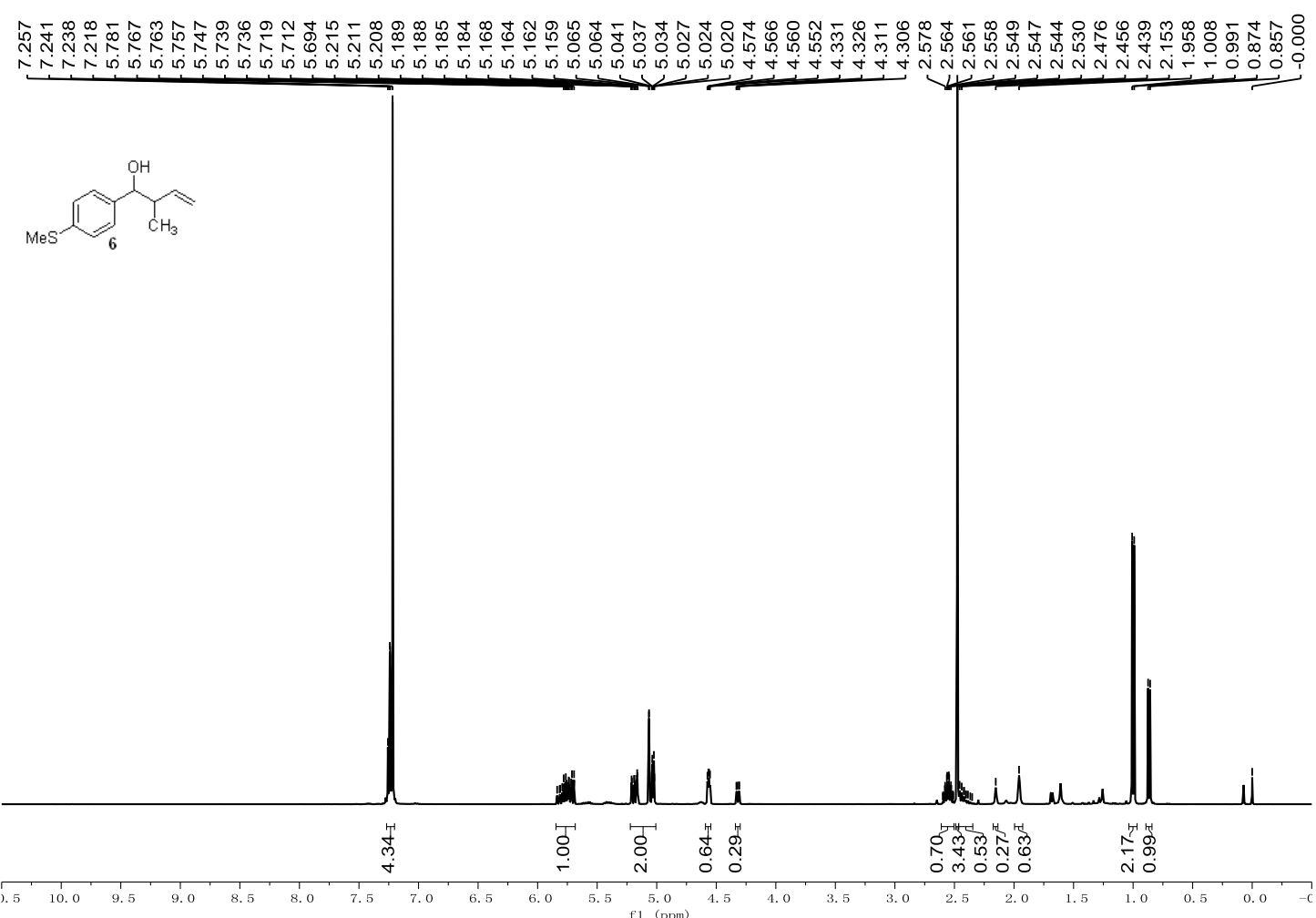

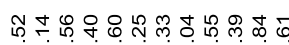

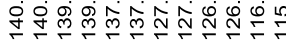

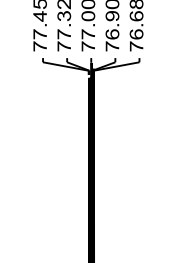

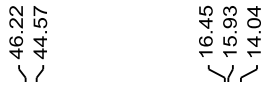
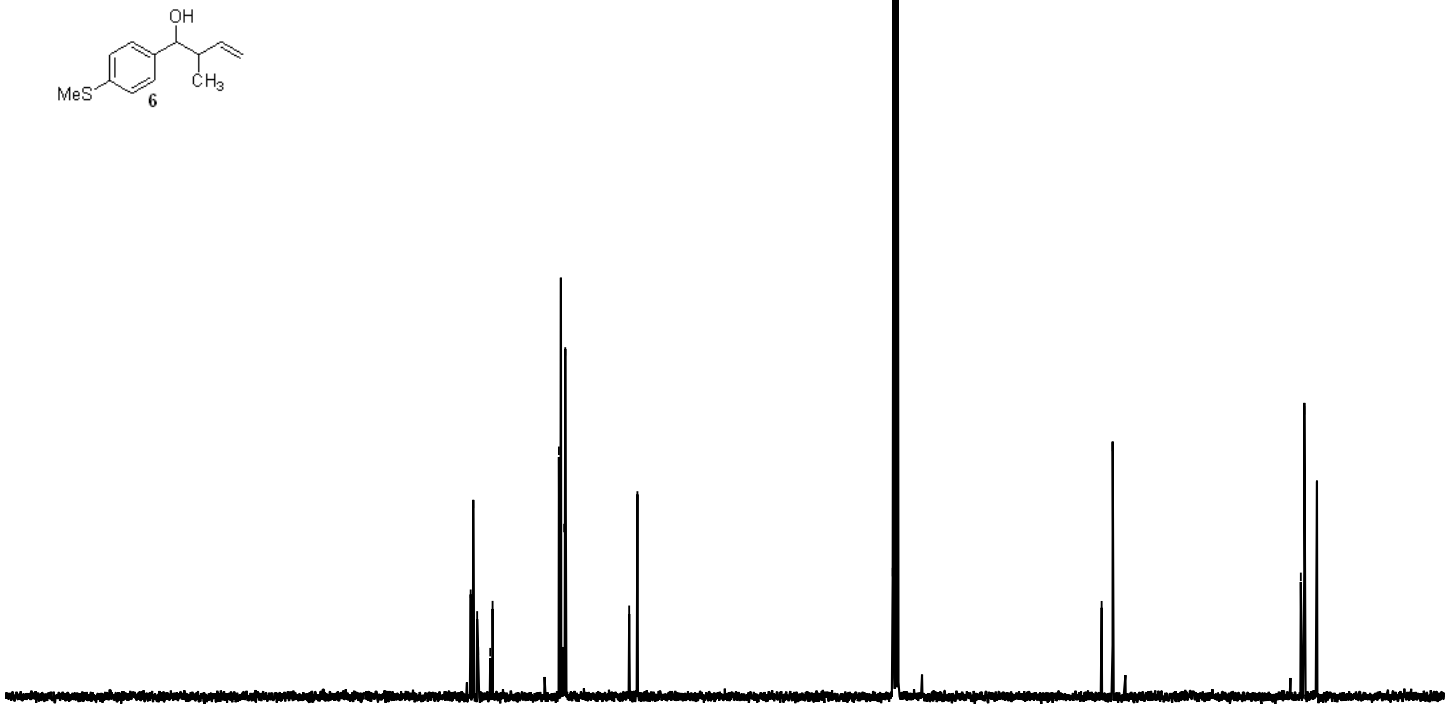

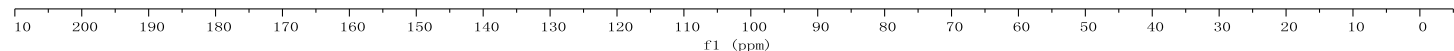



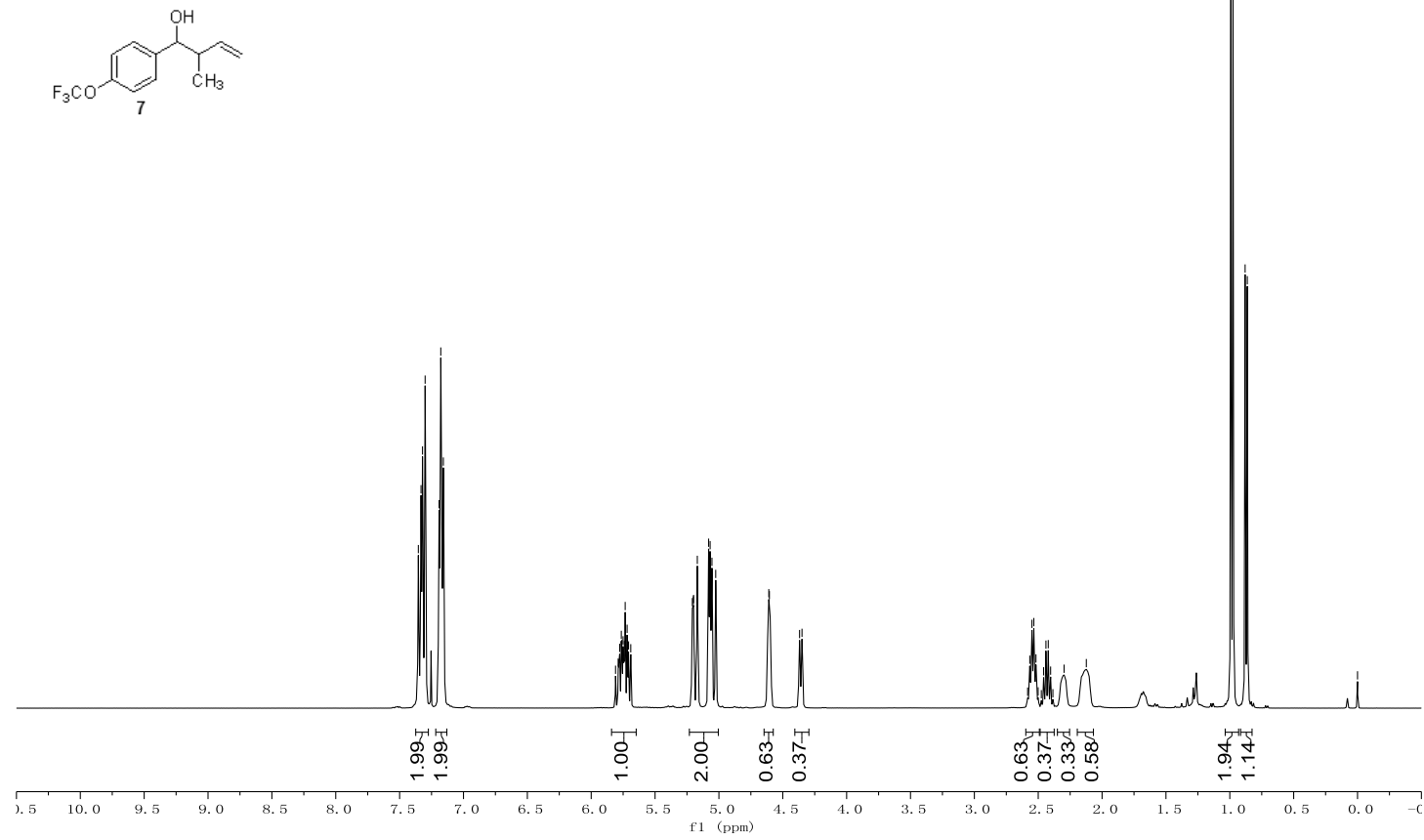

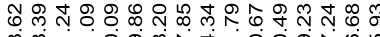
$\underbrace{\infty}$

m:

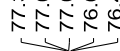

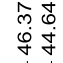

$\ddot{\infty}$

$\stackrel{00}{1}$
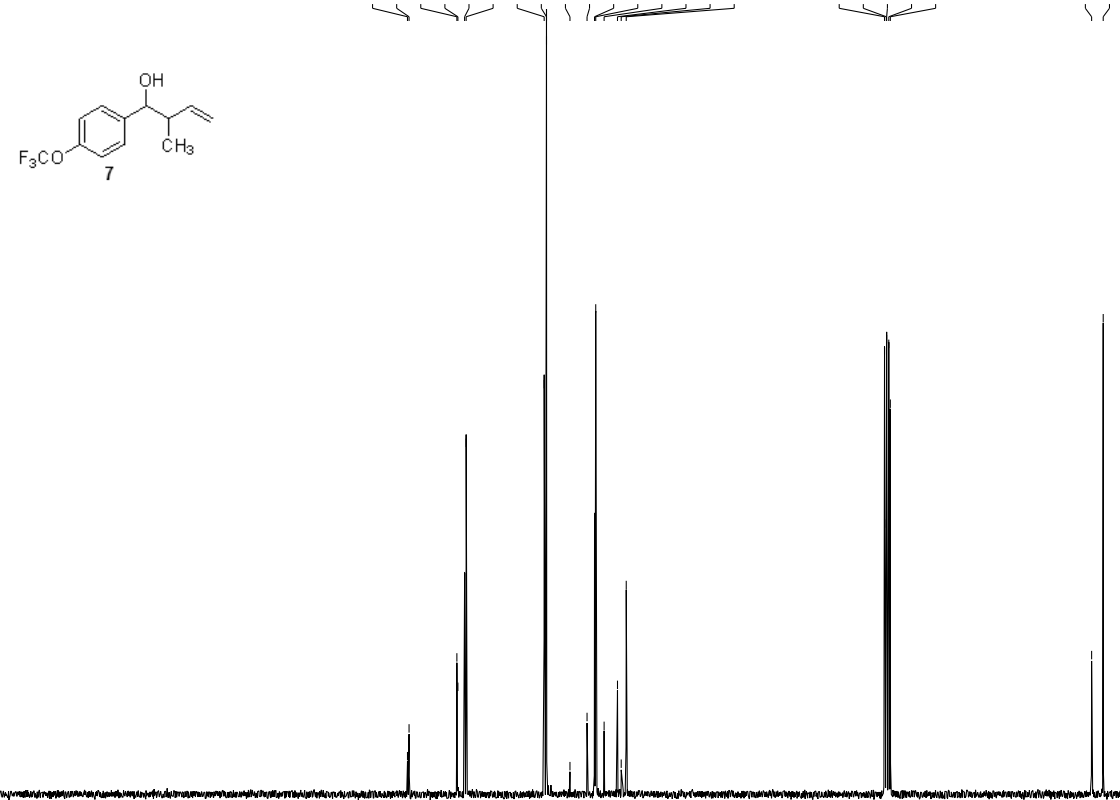

\begin{tabular}{llllllllllll}
\hline 10 & 200 & 190 & 180 & 170 & 160 & 150 & 140 & 130 & 120 & 110 & 100 \\
$\mathrm{f1}$ & $(\mathrm{ppm})$
\end{tabular} 


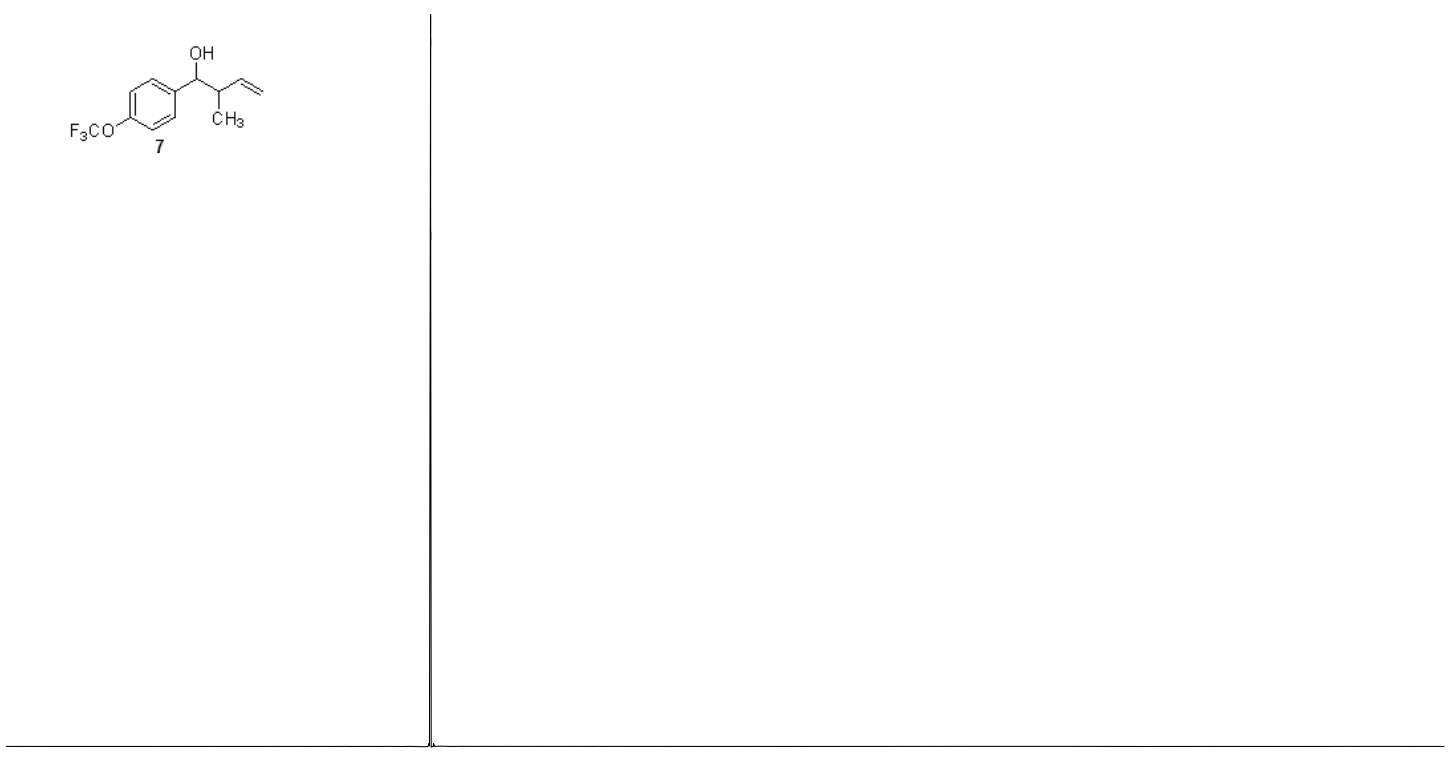

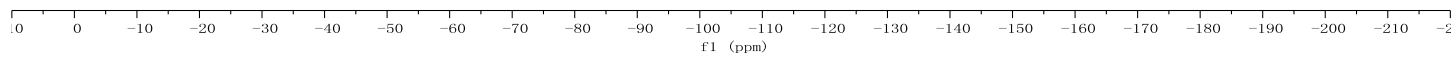




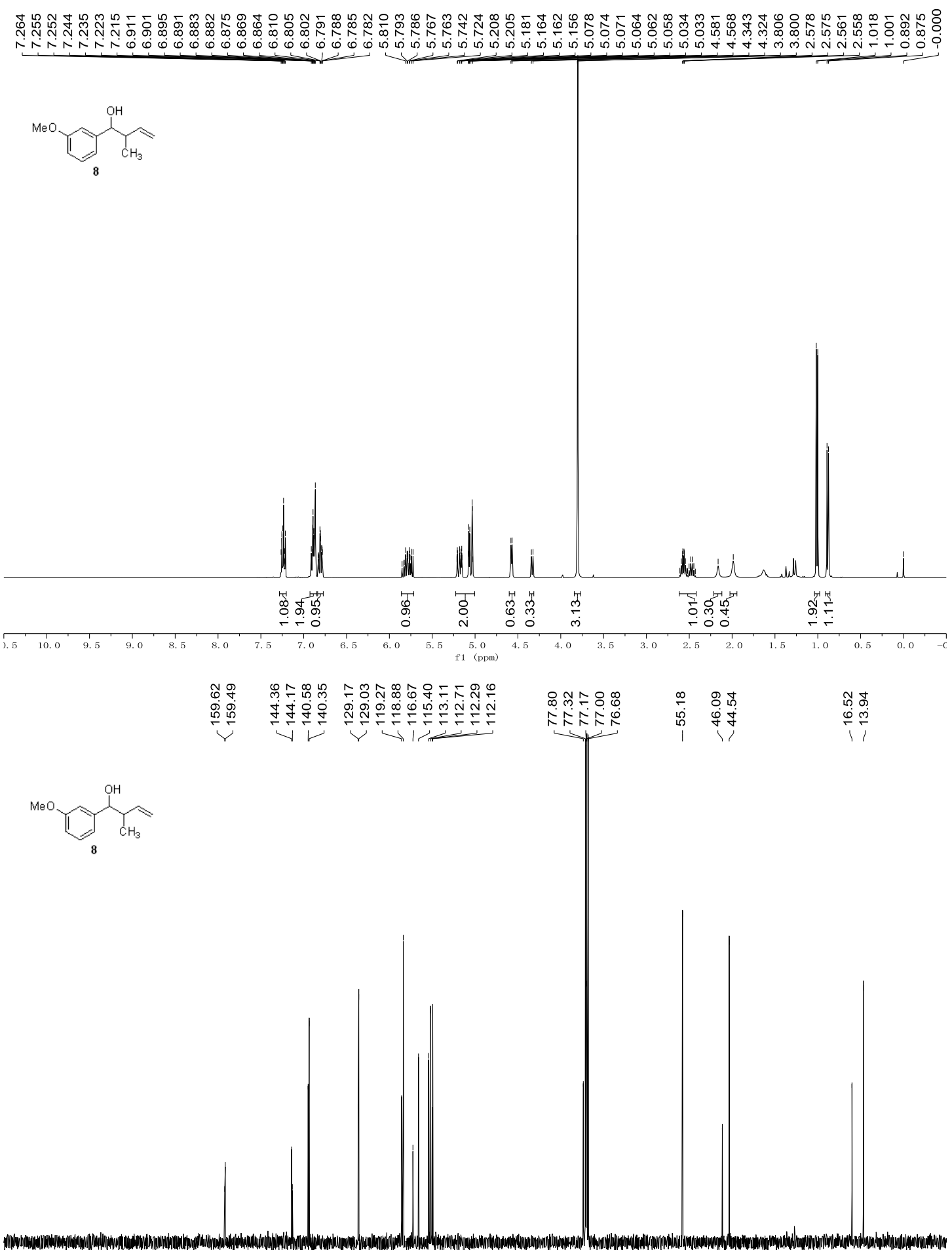

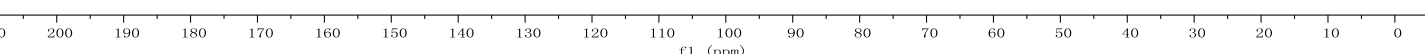


<smiles>C=CC(C)C(O)c1ccccc1[N+](=O)[O-]</smiles>

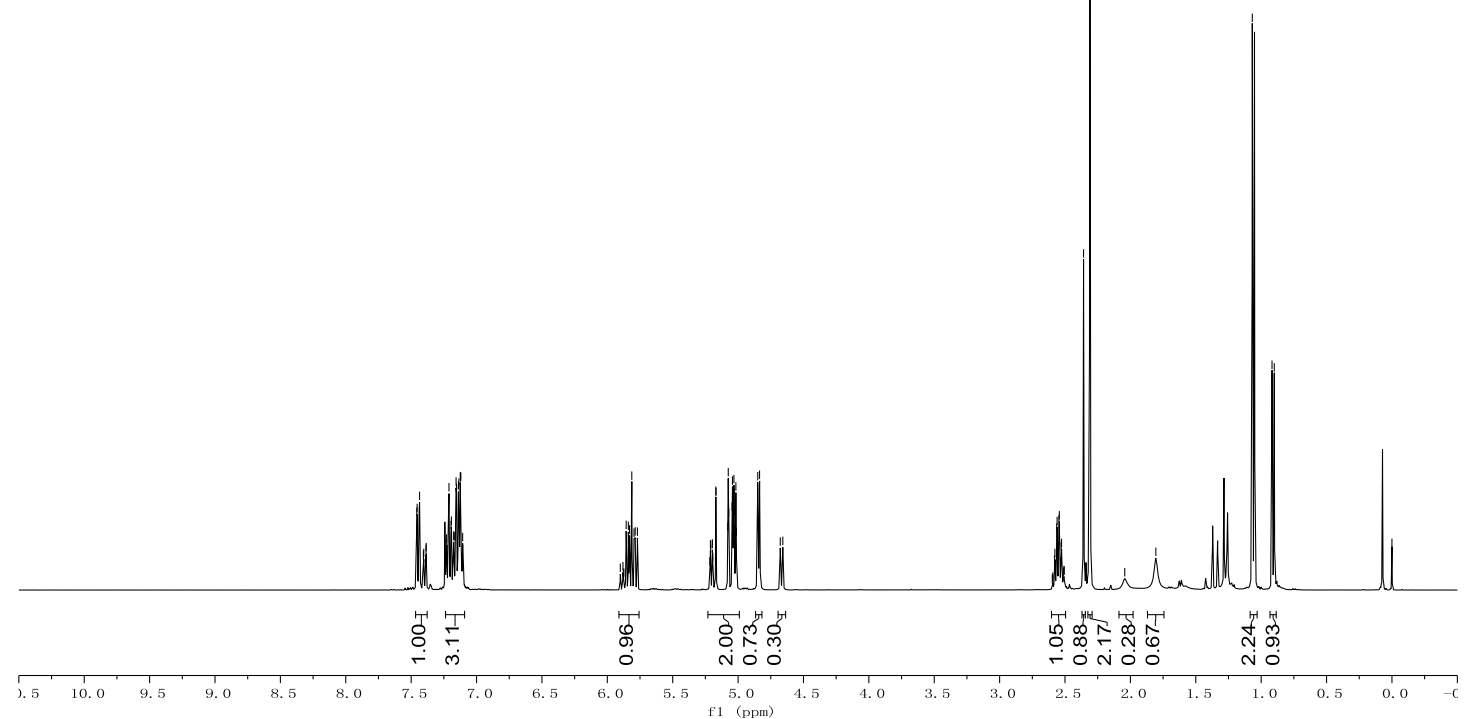

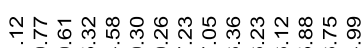

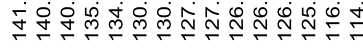

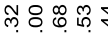

isisis

$\stackrel{2}{N}$

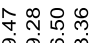

li

$\int_{\mathrm{CH}_{3}}^{\mathrm{OH}}$

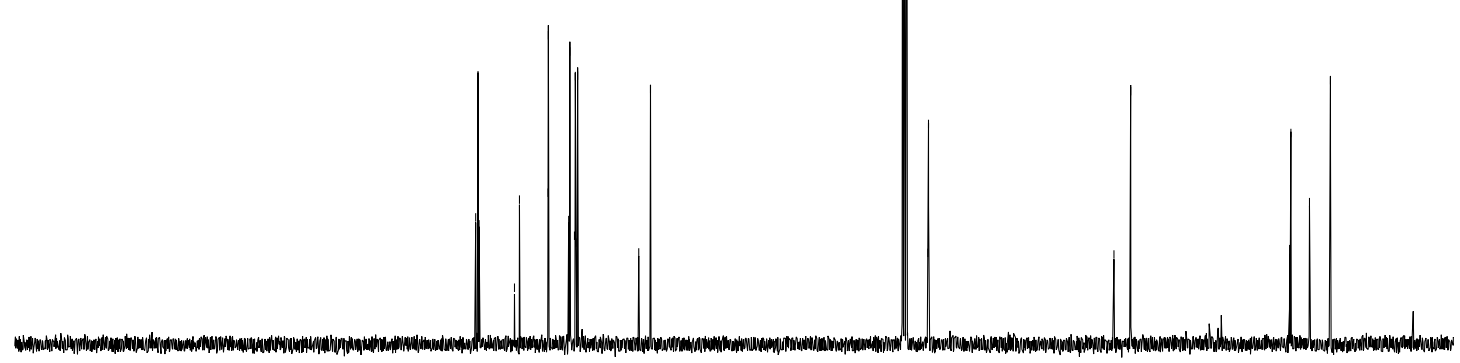

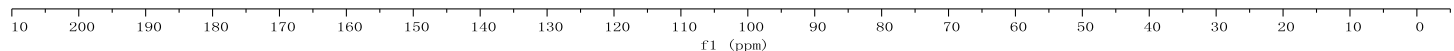




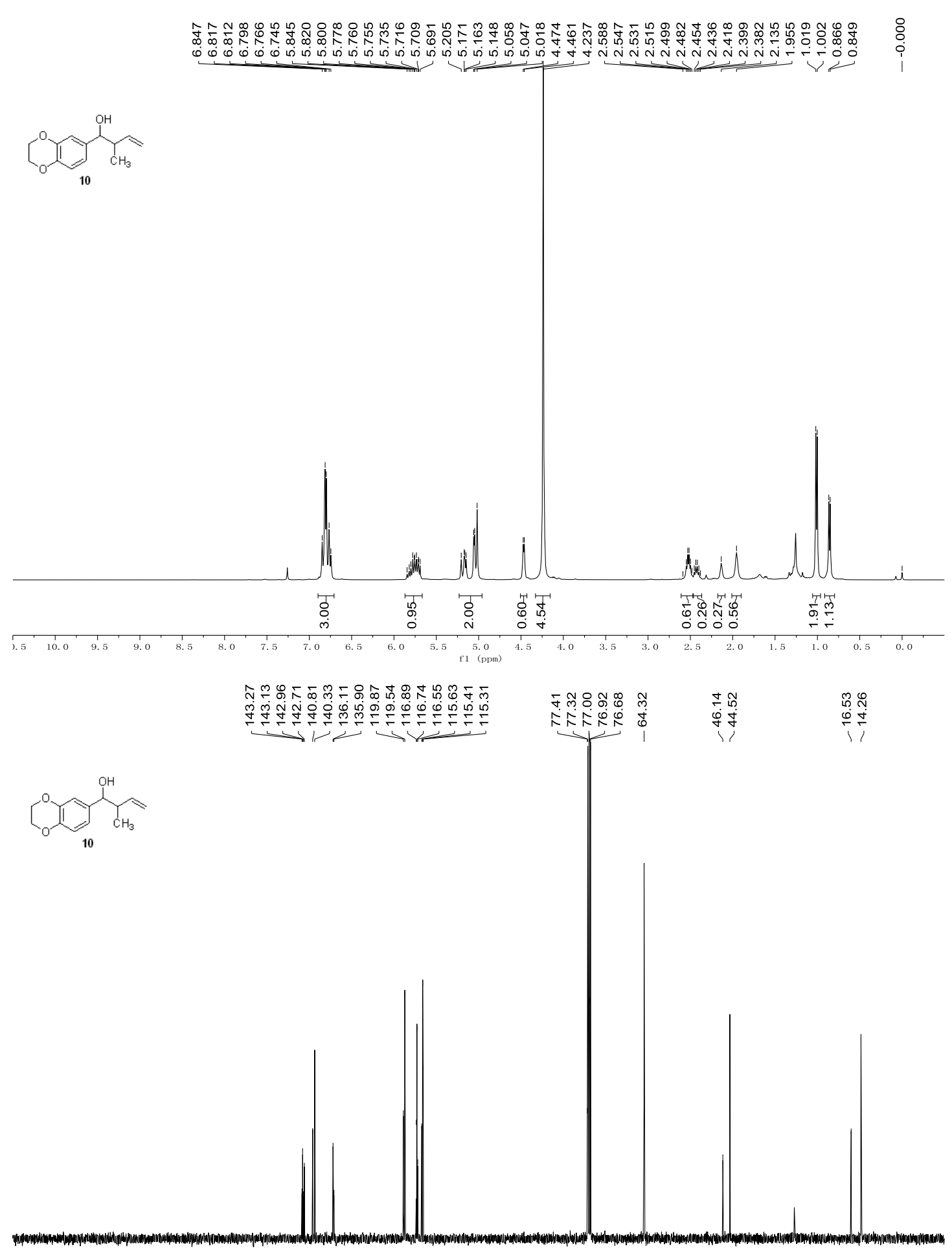

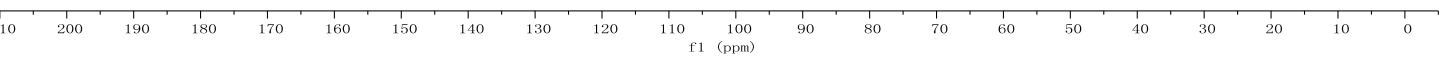




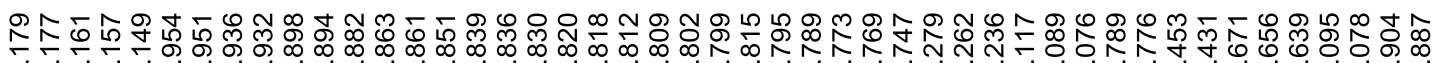

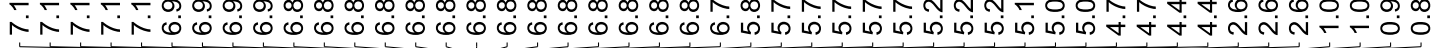<smiles>C=CC(C)C(O)c1ccccc1O</smiles>

11

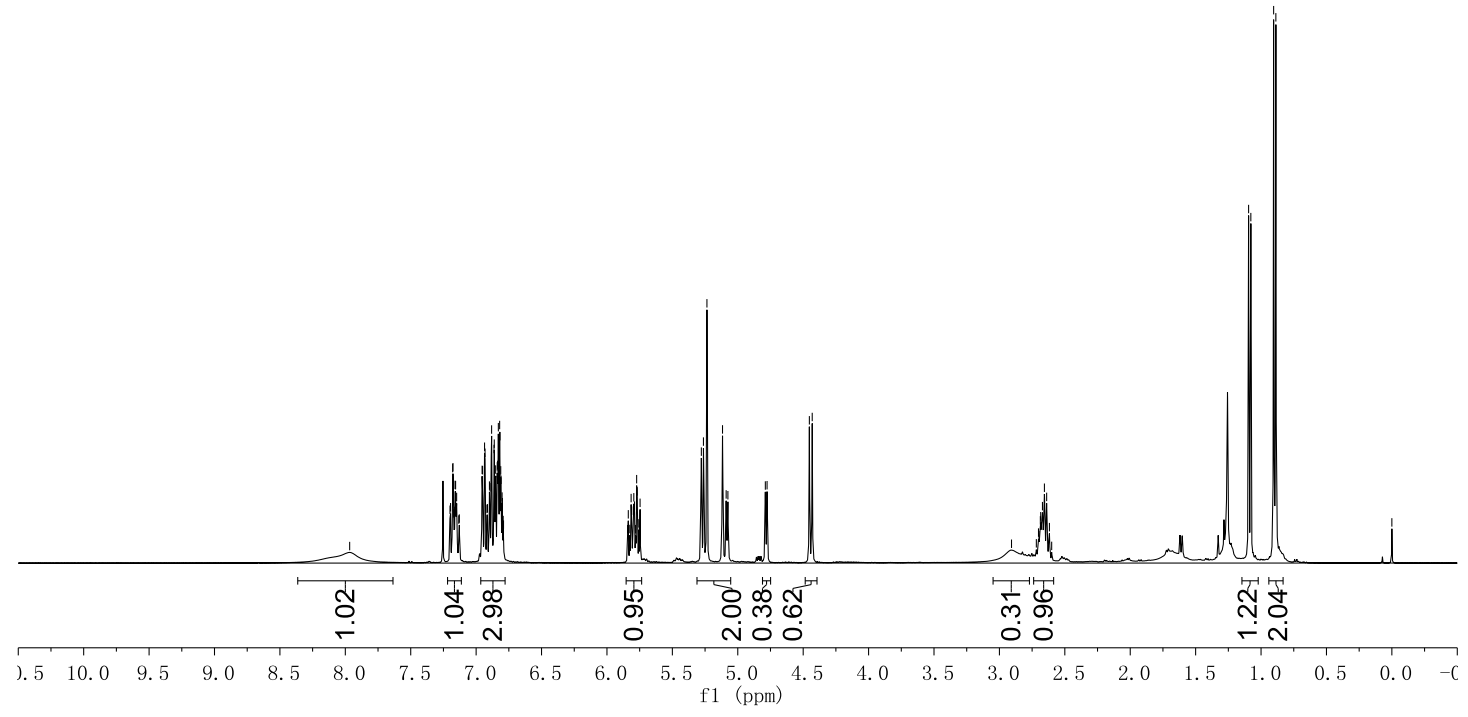

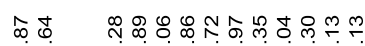

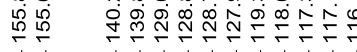

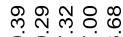

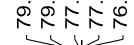

mơ

$\stackrel{\infty}{\infty} \underset{0}{\infty}$

i

$\underbrace{\mathrm{OH}}_{11}$

11

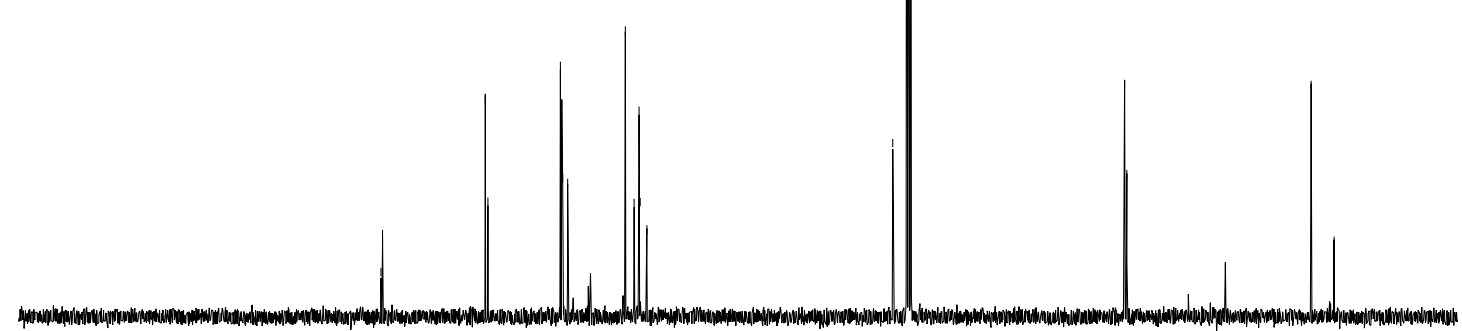

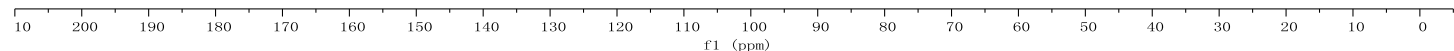




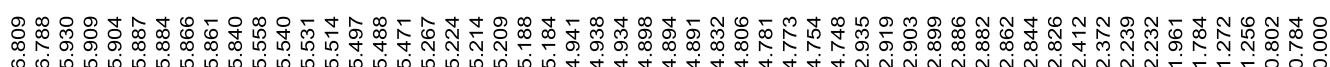

0
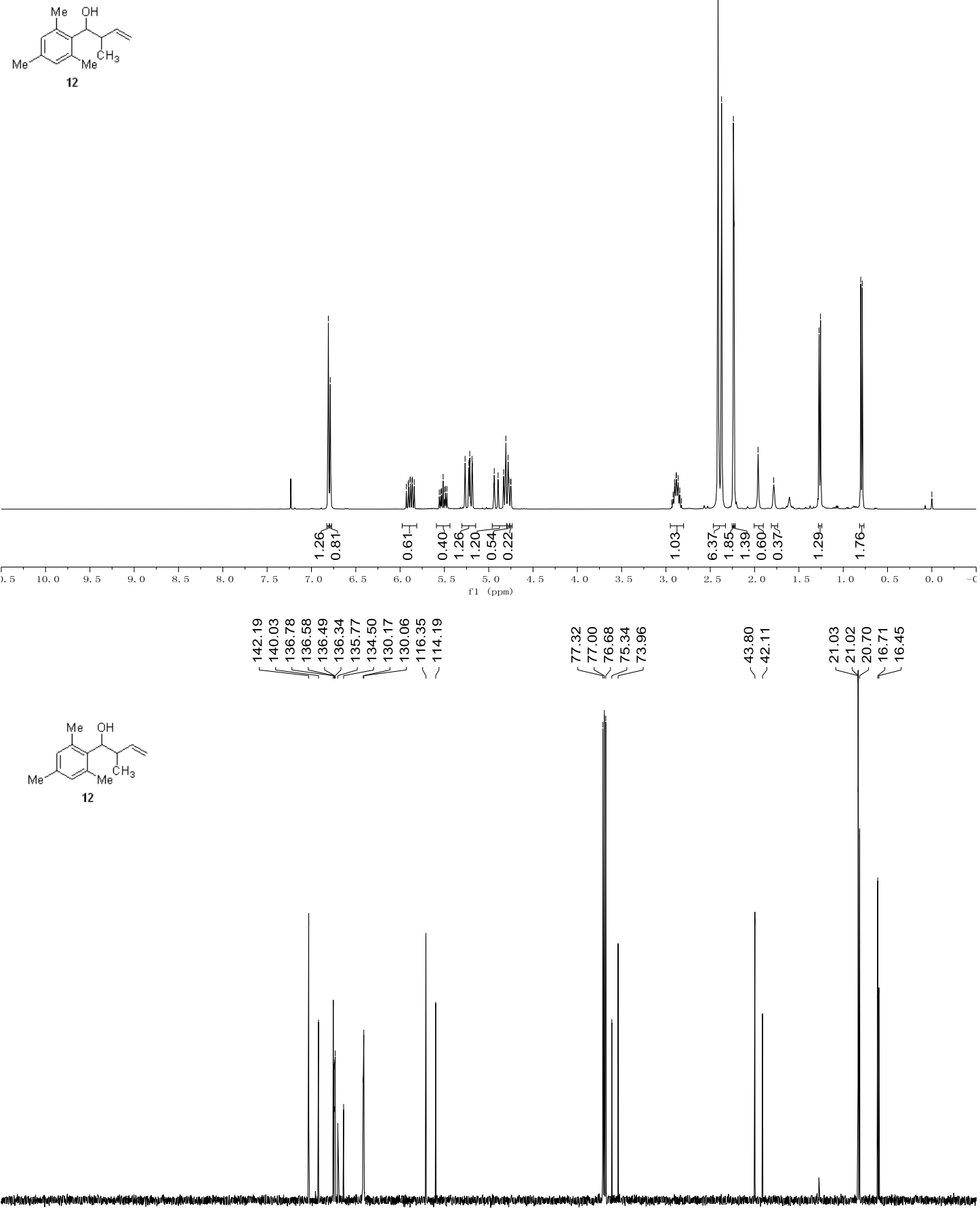

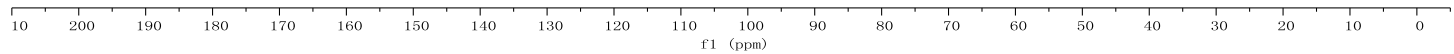




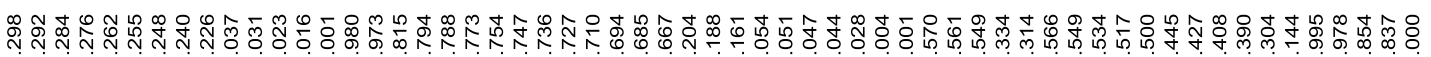

N
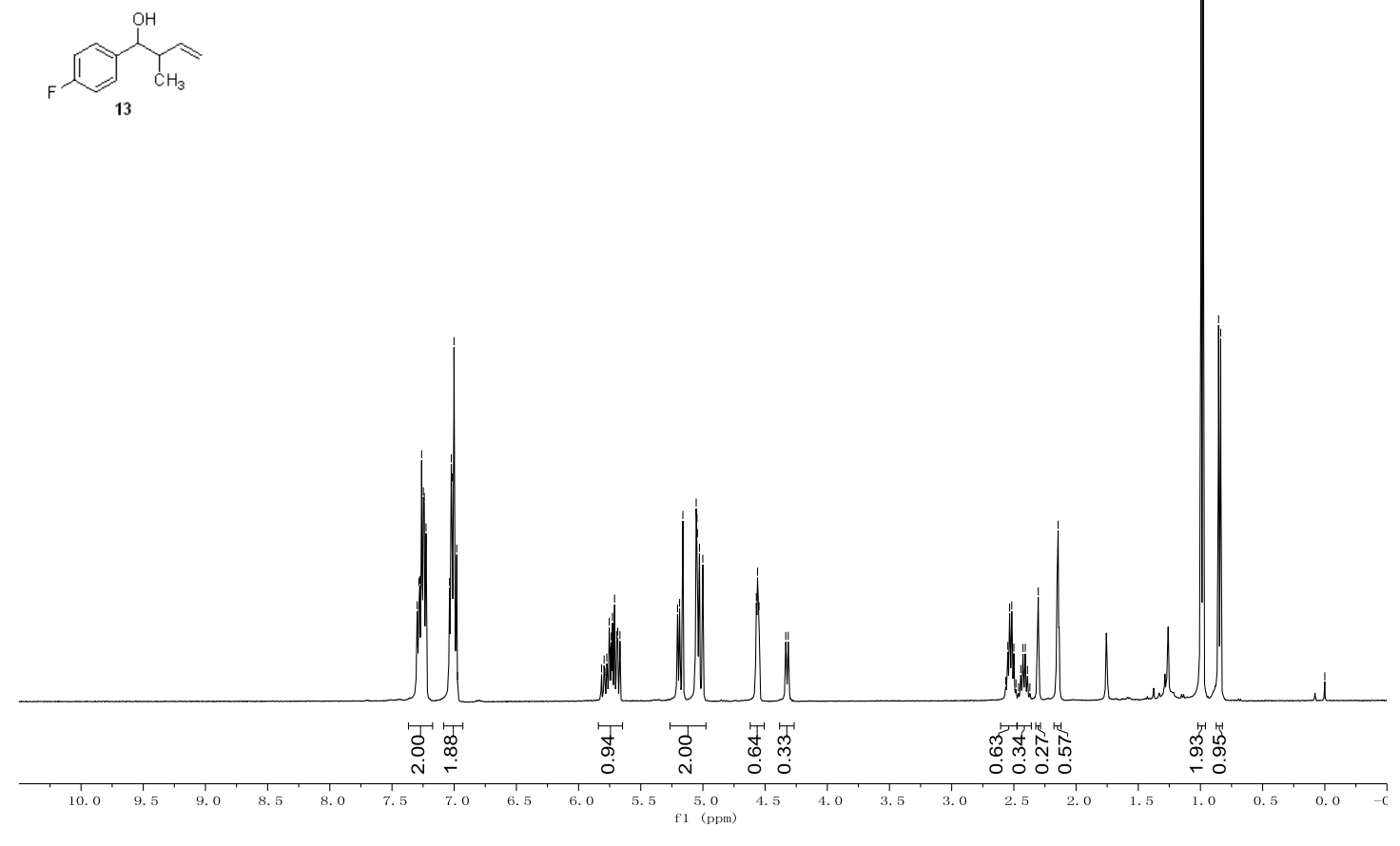

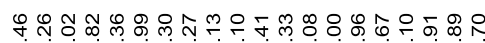

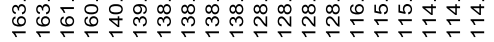

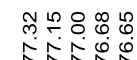

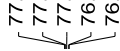

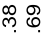

象 to

$i$

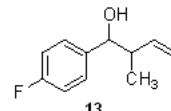

13
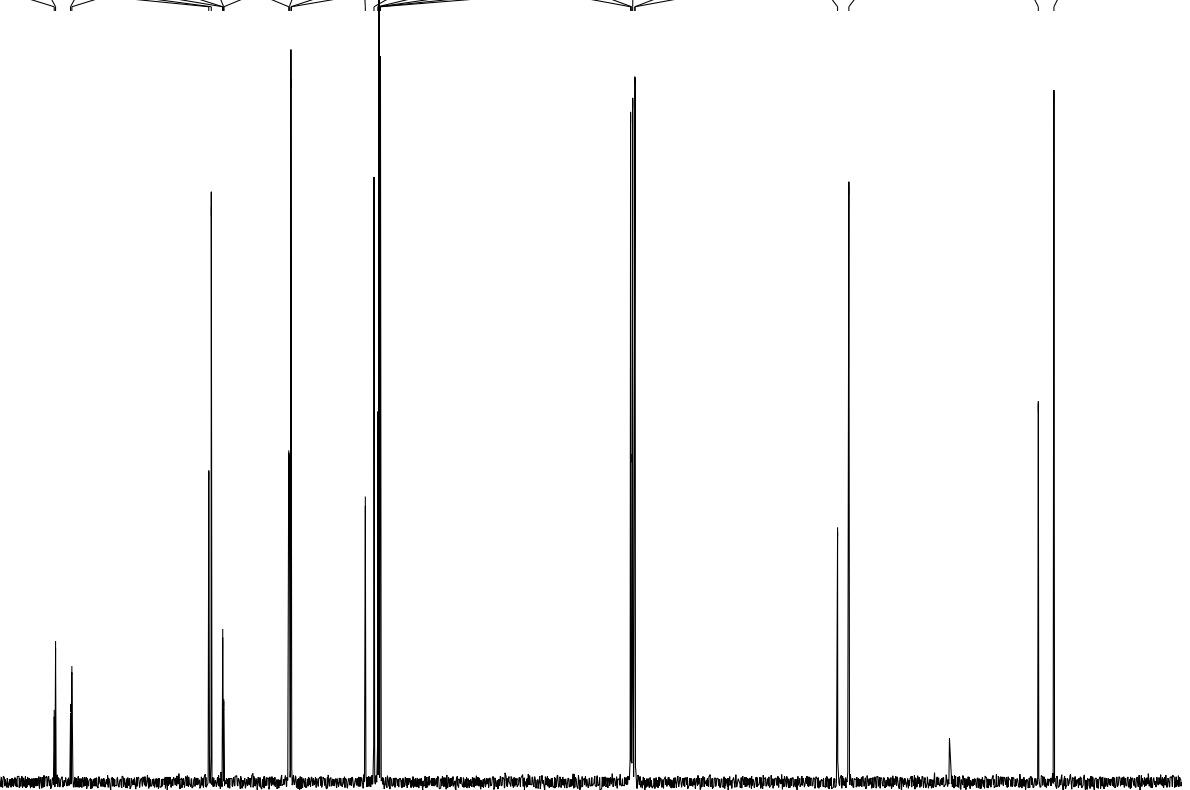

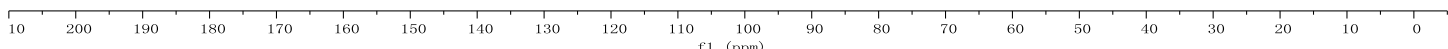


$\overbrace{\mathrm{CH}_{3}}^{\mathrm{OH}}$

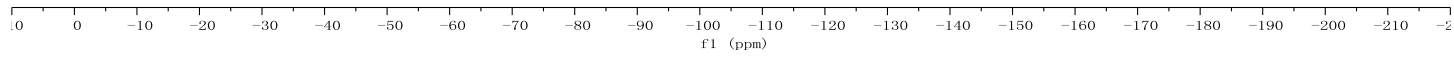



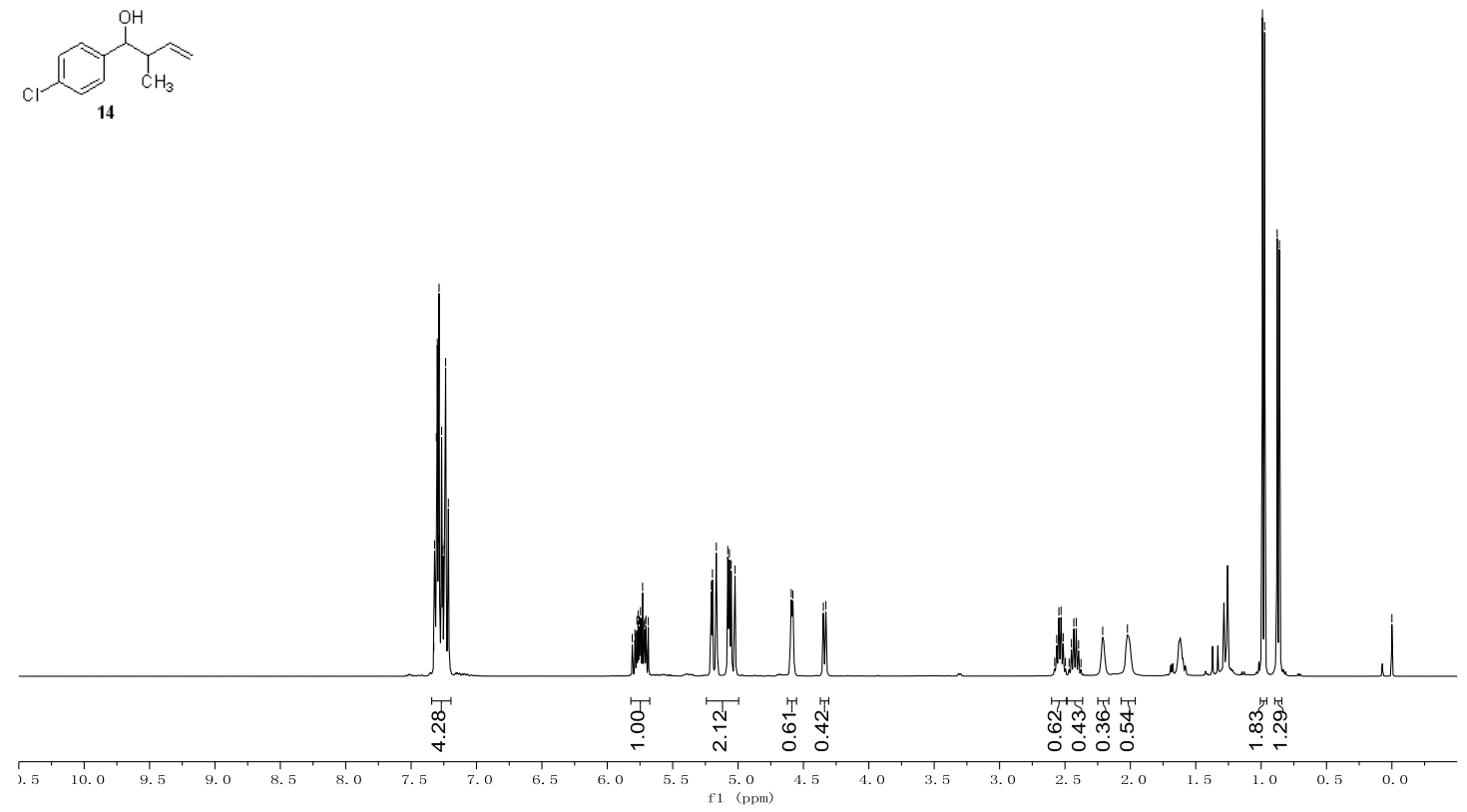

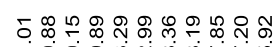

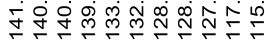

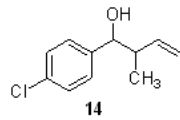

लำ

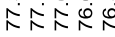

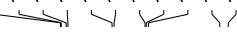

14
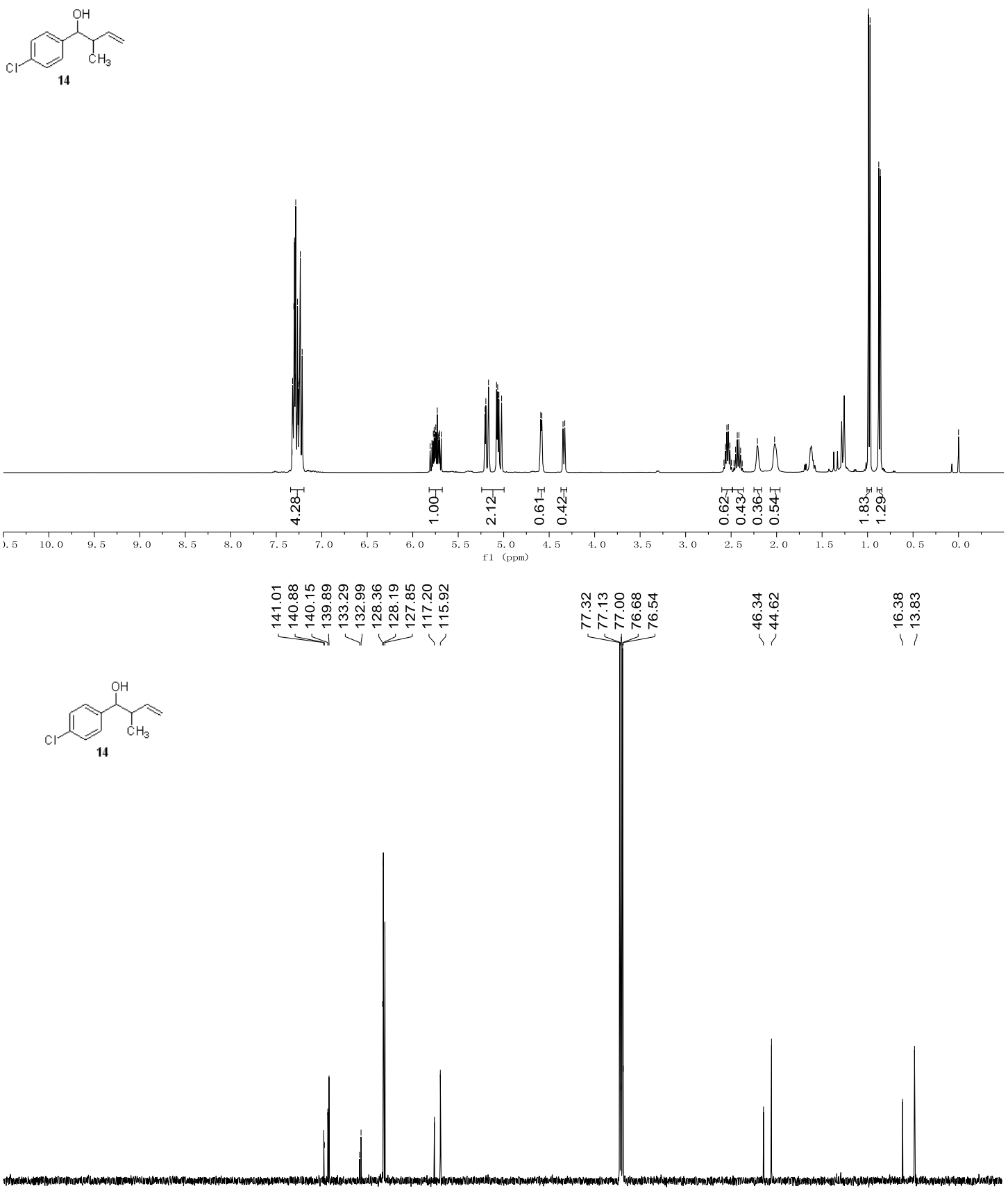

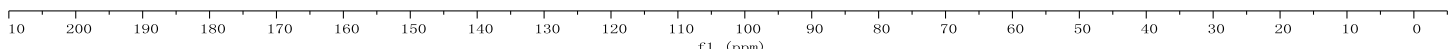




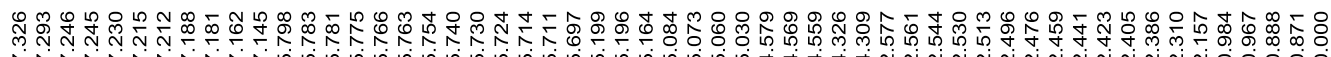

$\int_{15}^{\mathrm{OH}}$
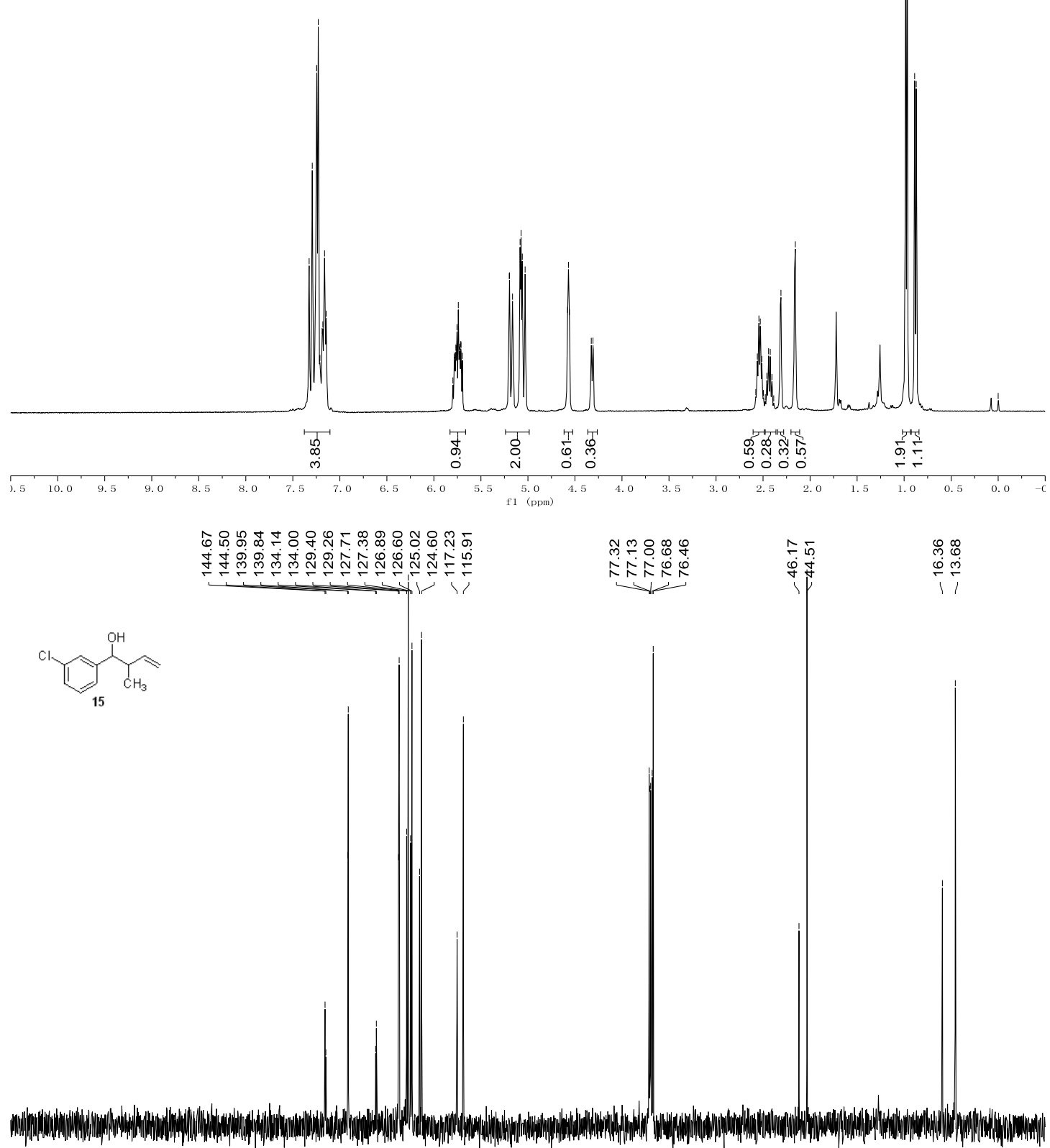

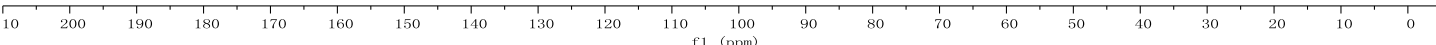


<smiles>C=CC(C)C(O)c1ccc(F)cc1</smiles>

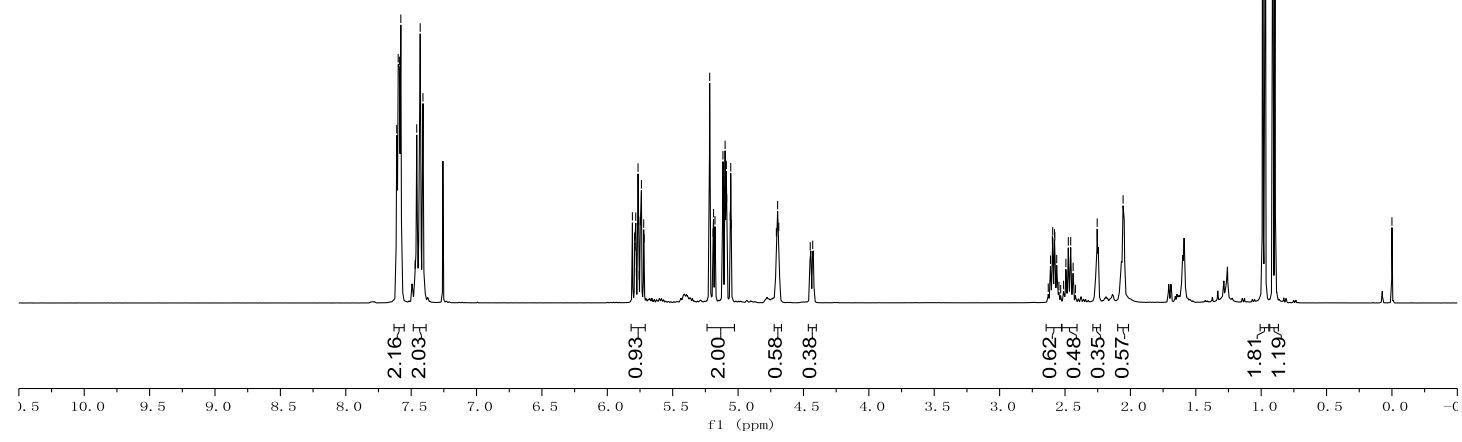

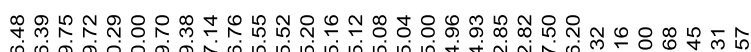

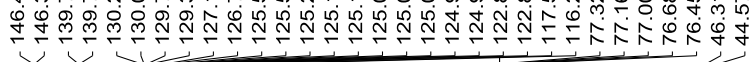
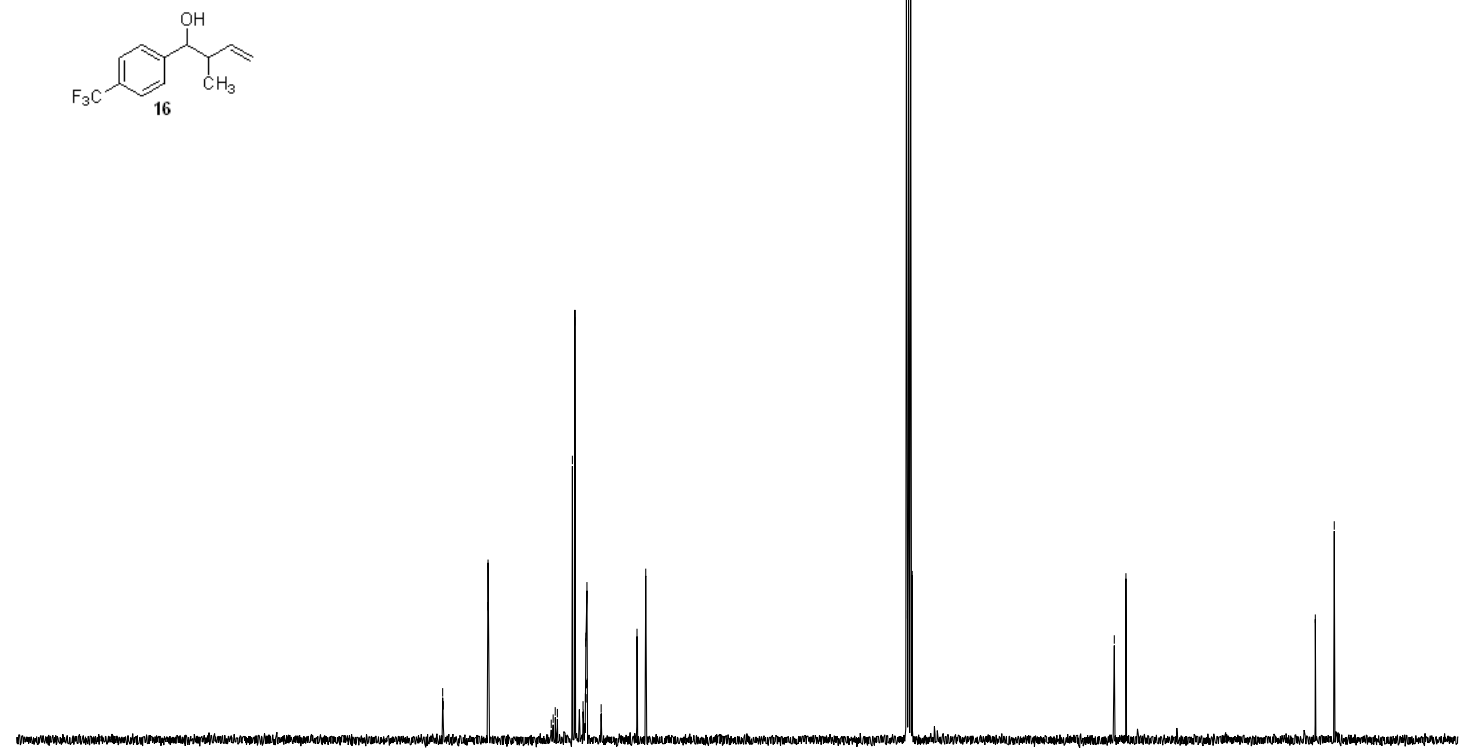

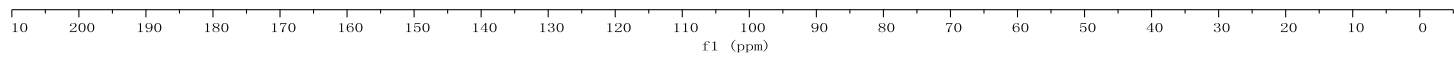



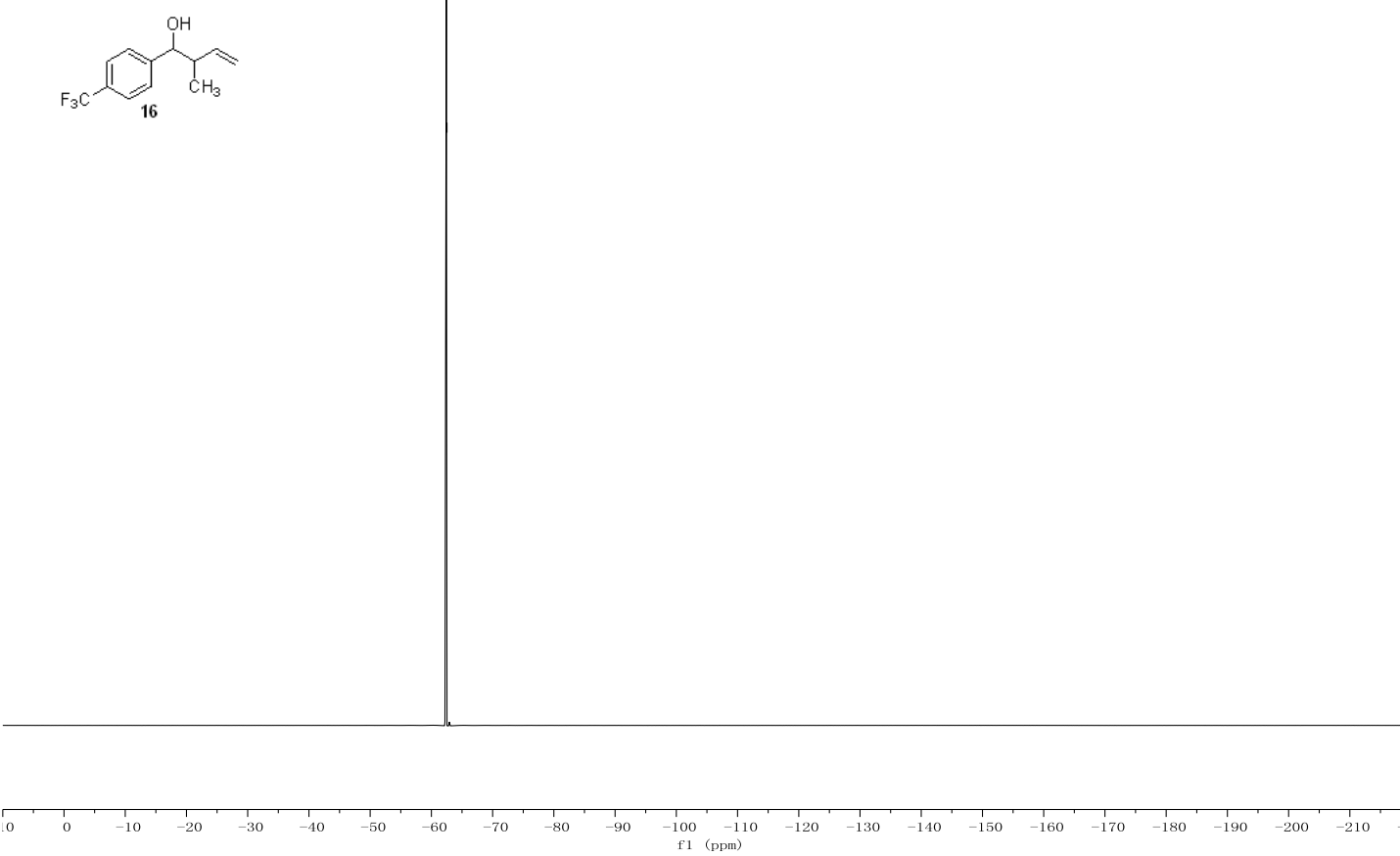


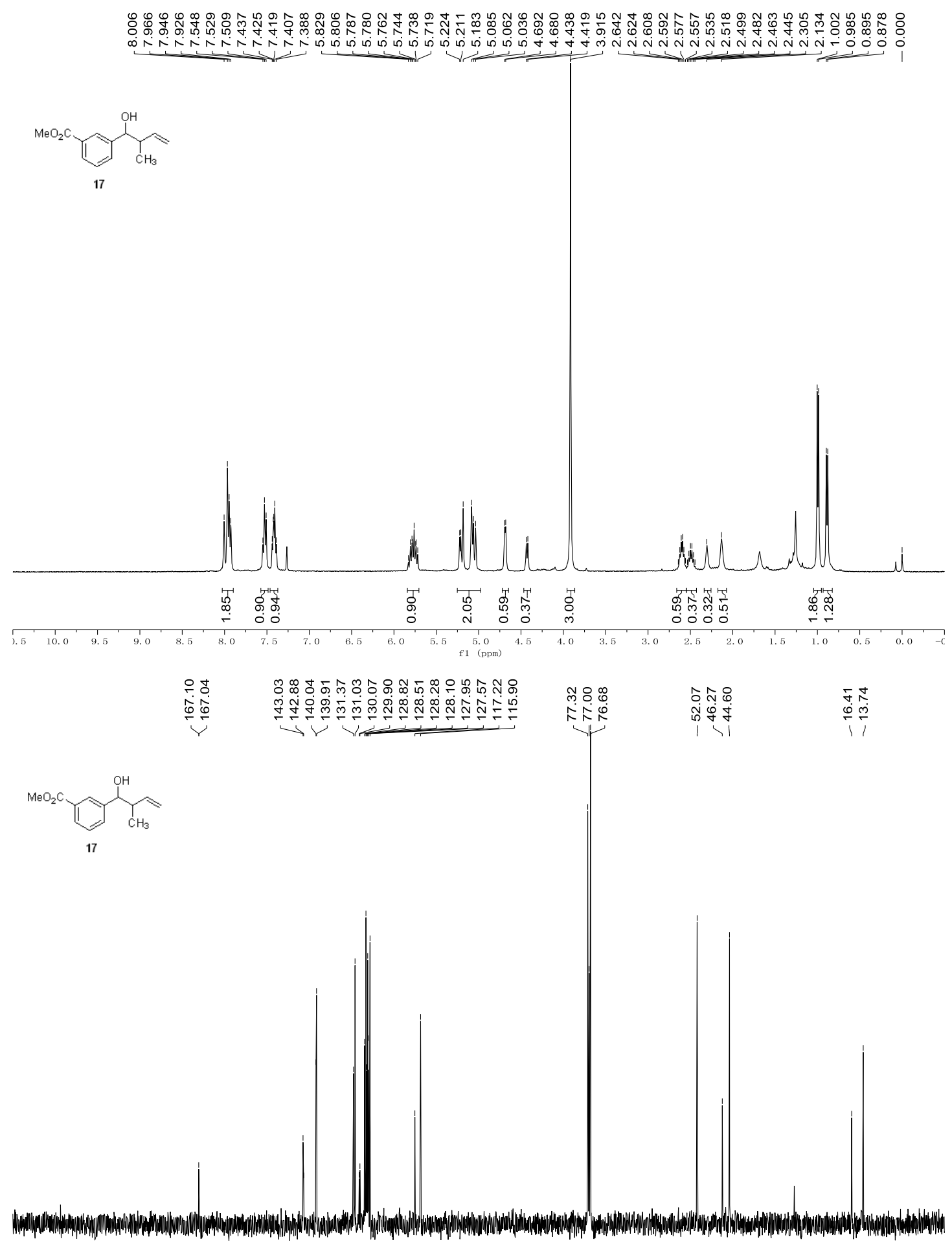

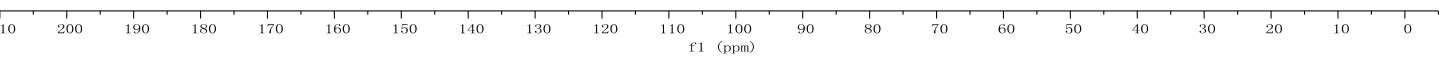




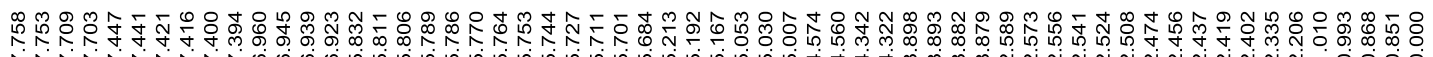

$\mathrm{MeO}_{18}^{\mathrm{OH}}$

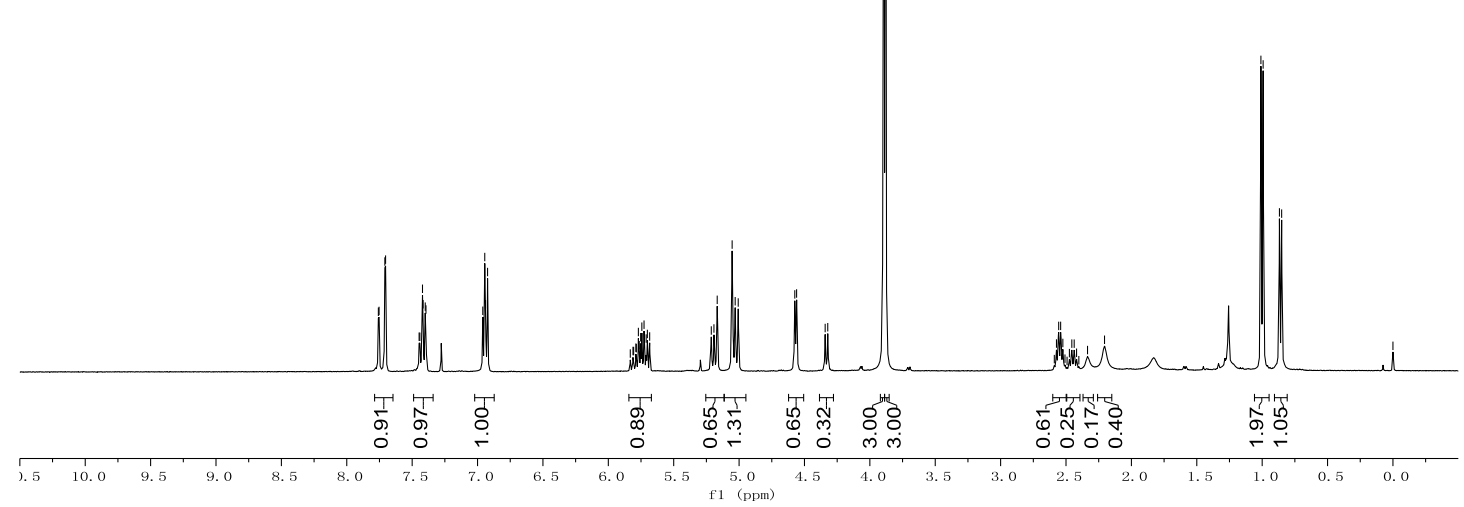

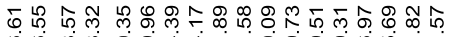

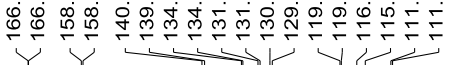



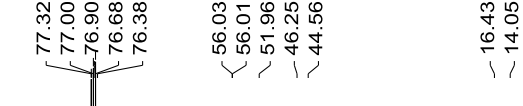

Nin

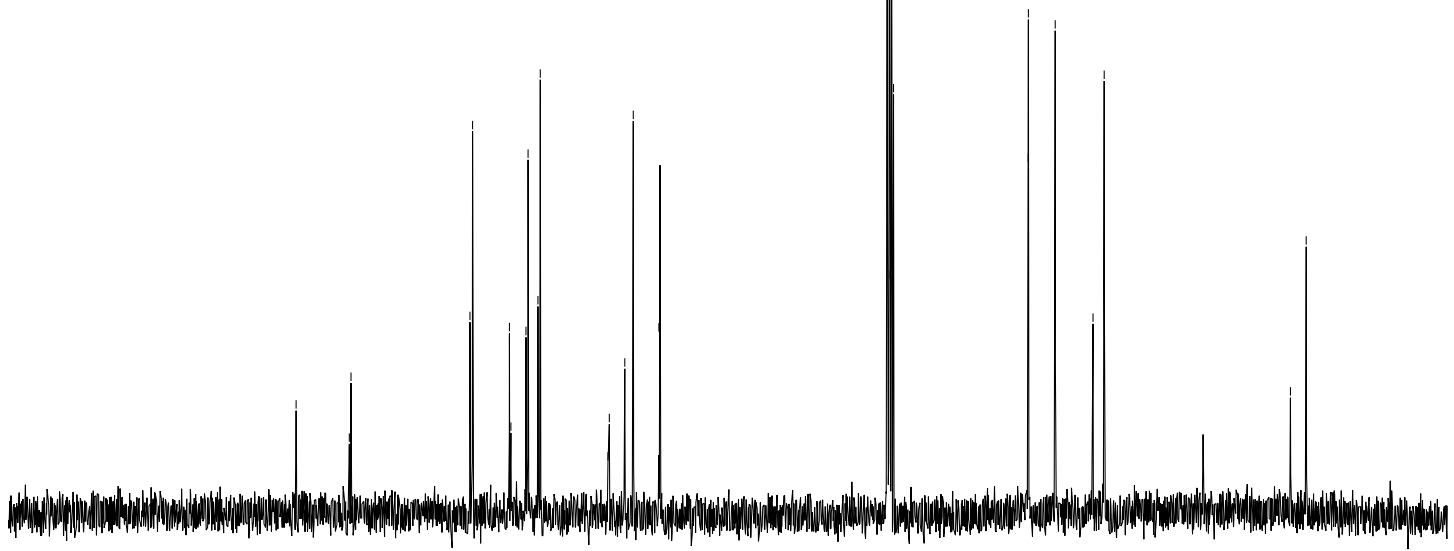

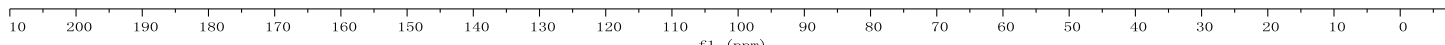




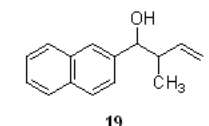

19
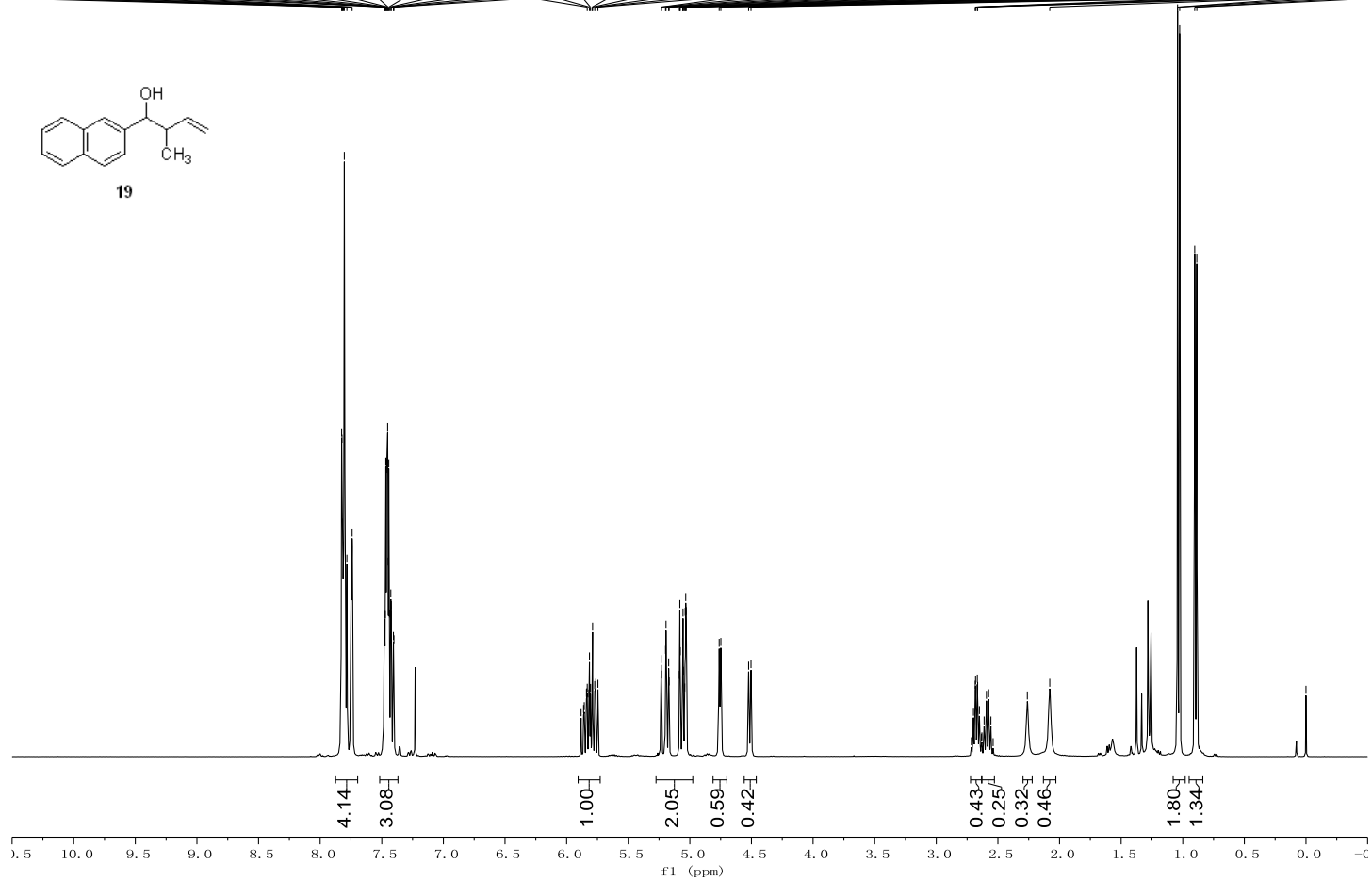

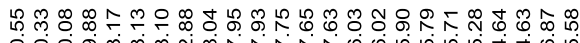

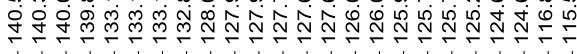
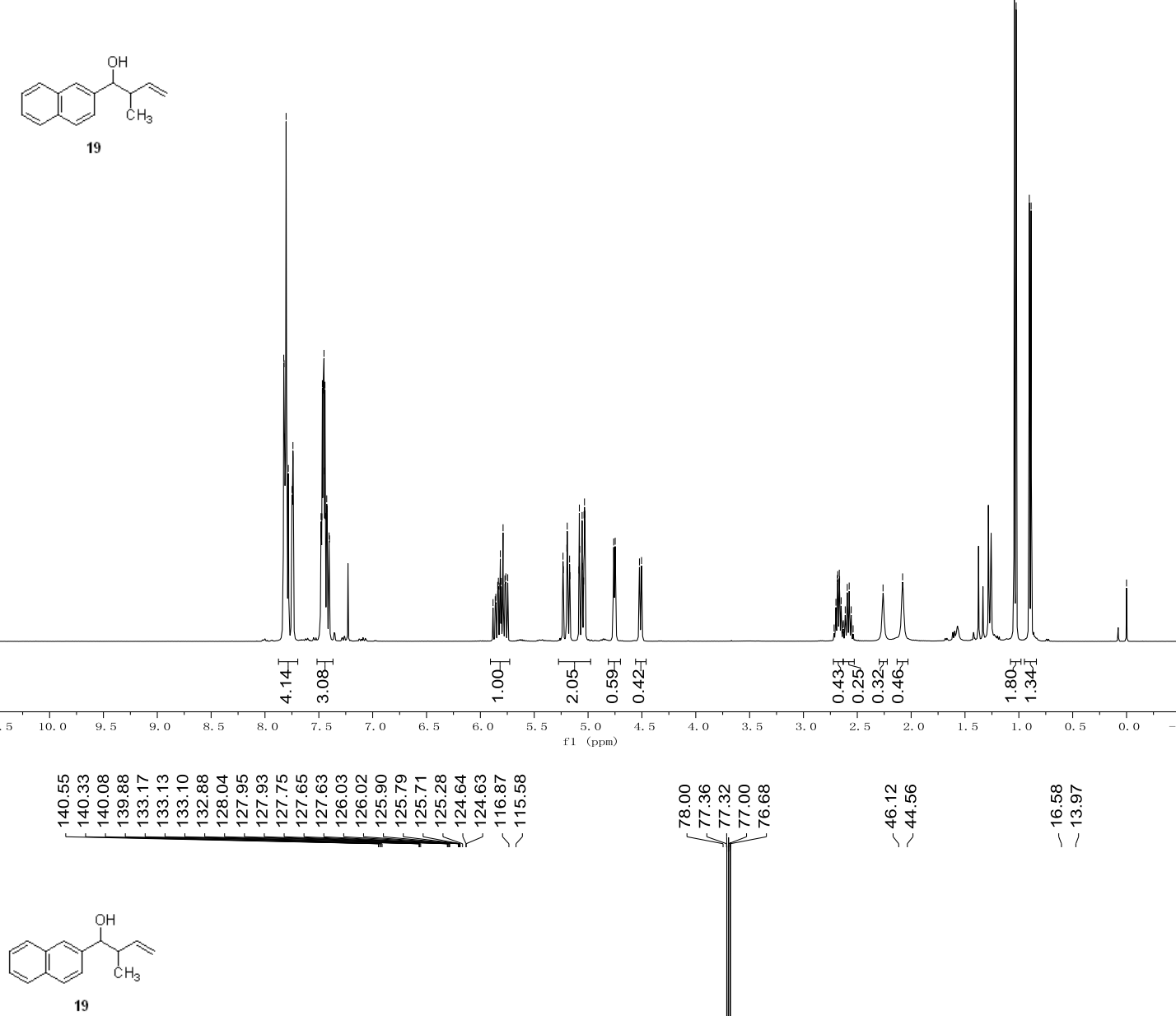

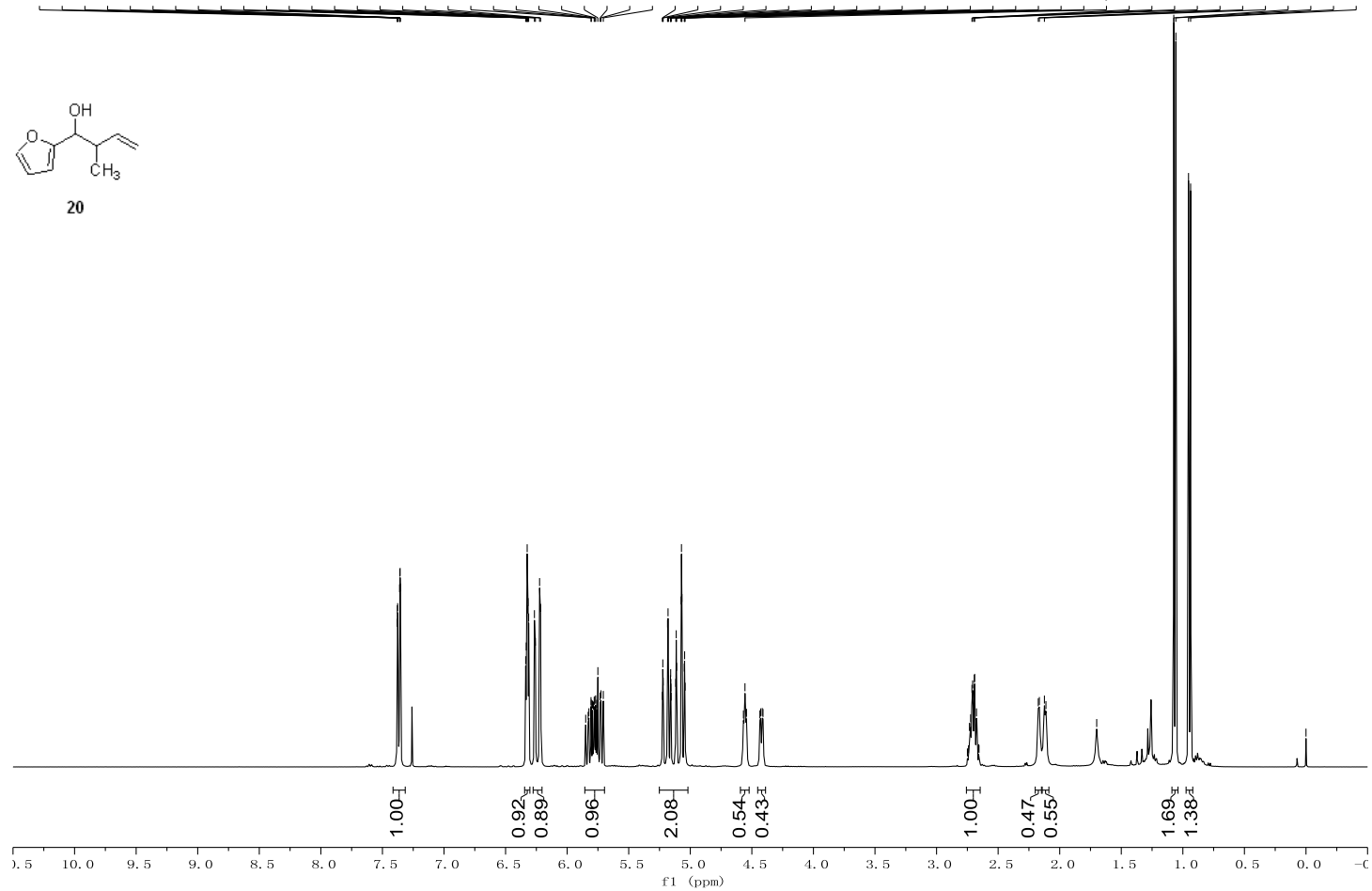

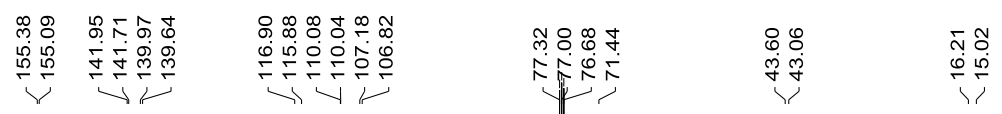

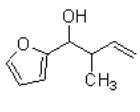

20
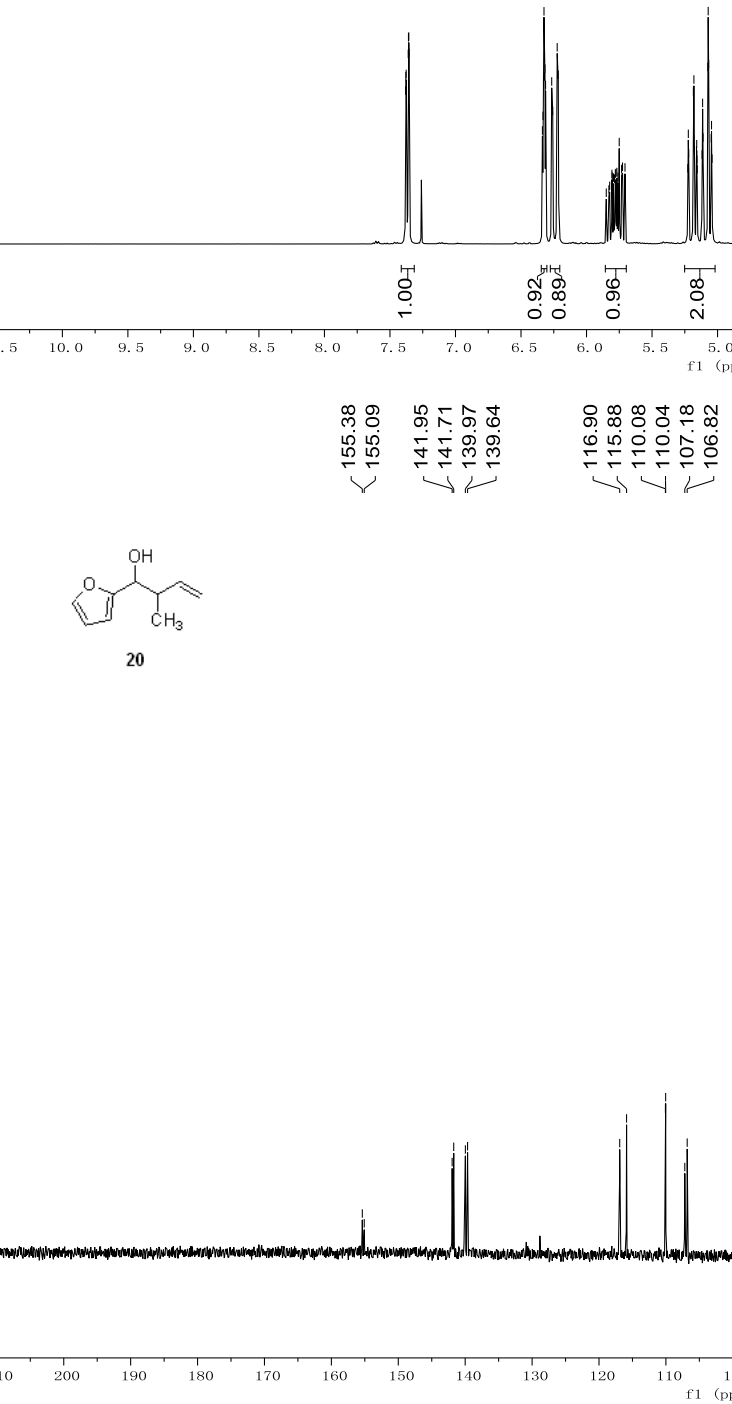


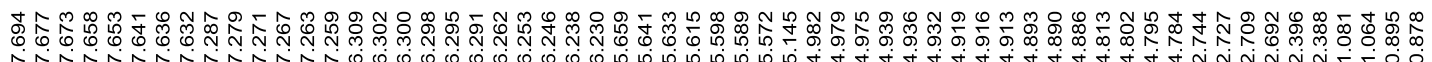
身

21

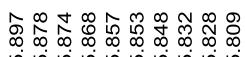

ம่

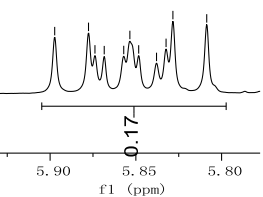

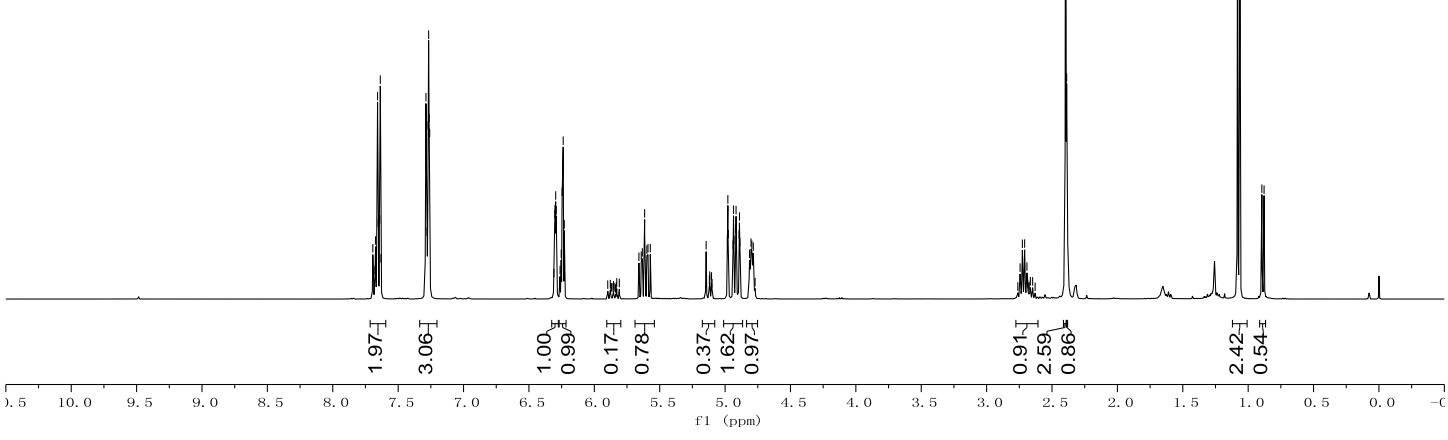

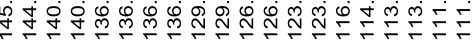

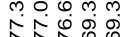

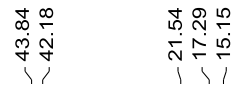

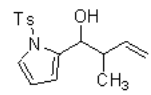

Y

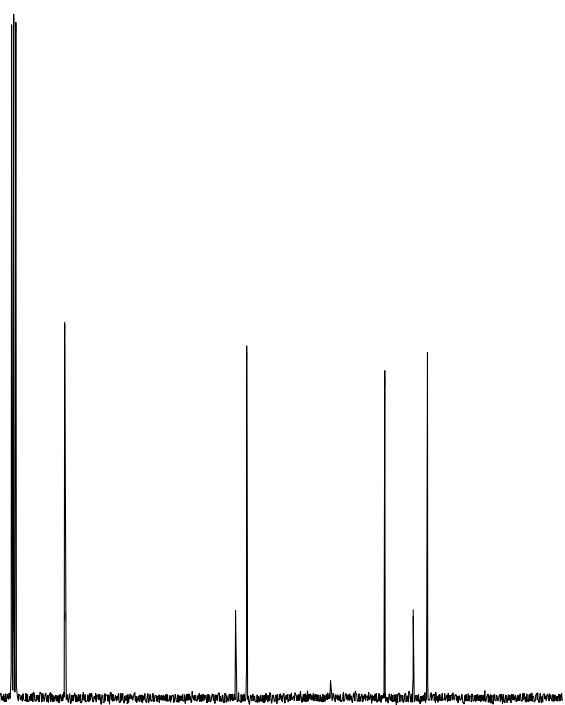

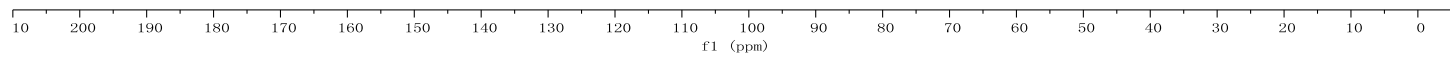




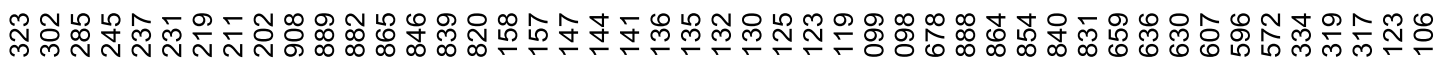

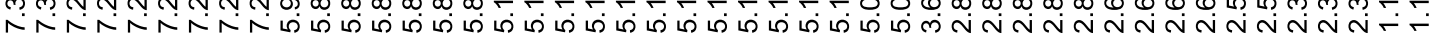

$\overbrace{22}^{\mathrm{O}}$
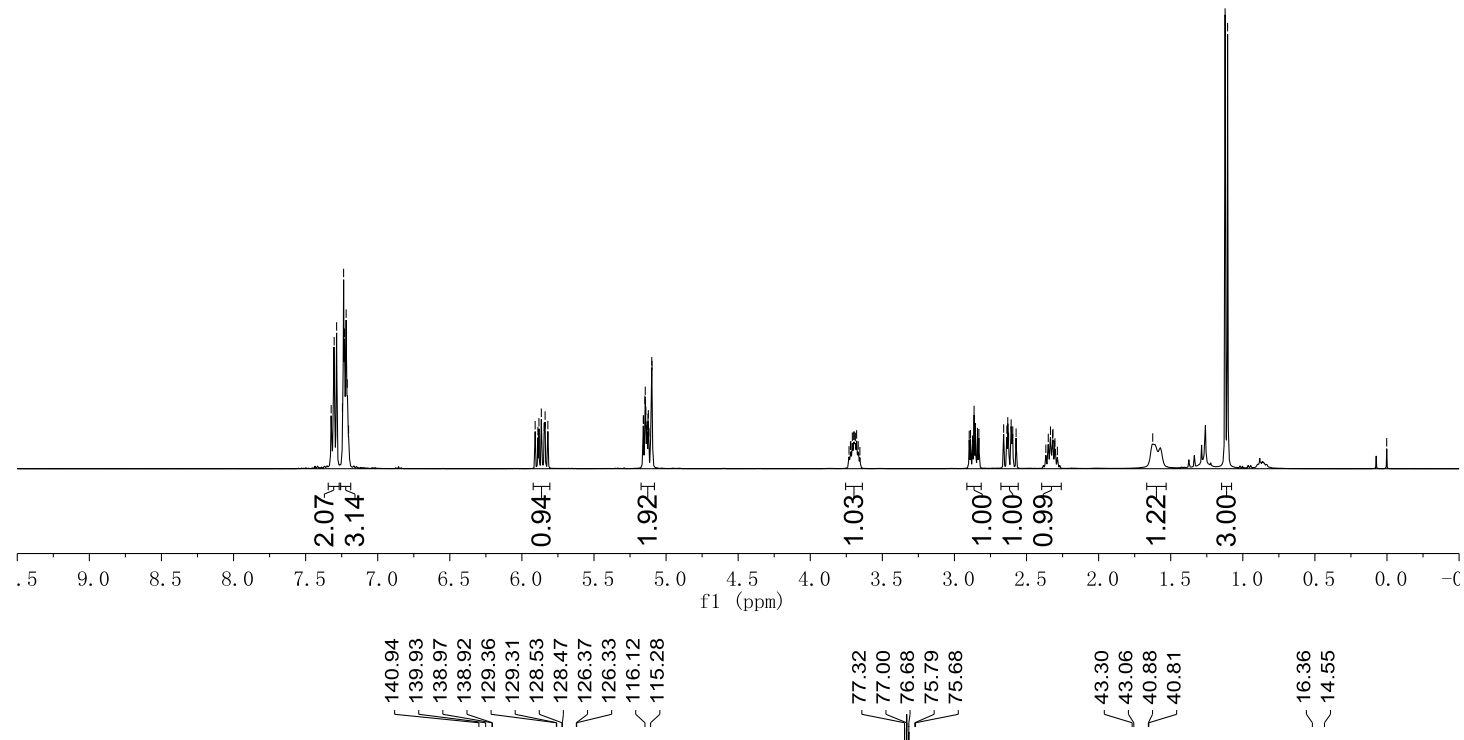

$\approx \underbrace{\mathrm{OH}}_{22} \curvearrowright$

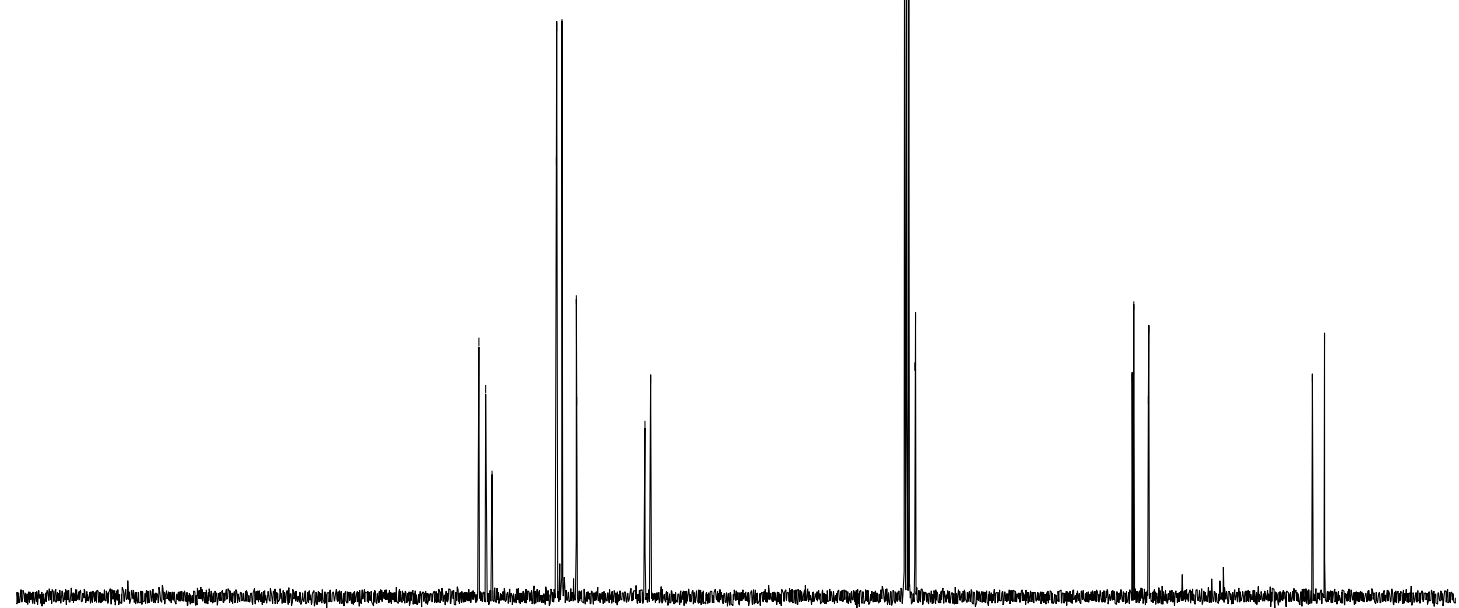

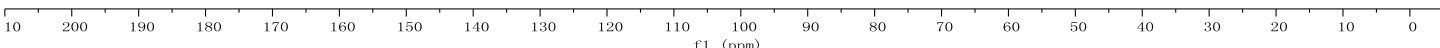




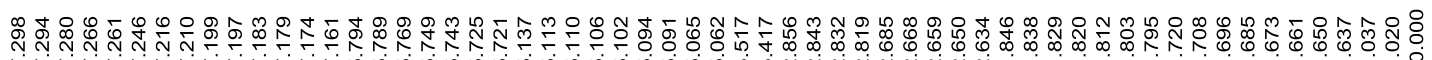

$\underbrace{\mathrm{OH}}_{23}$

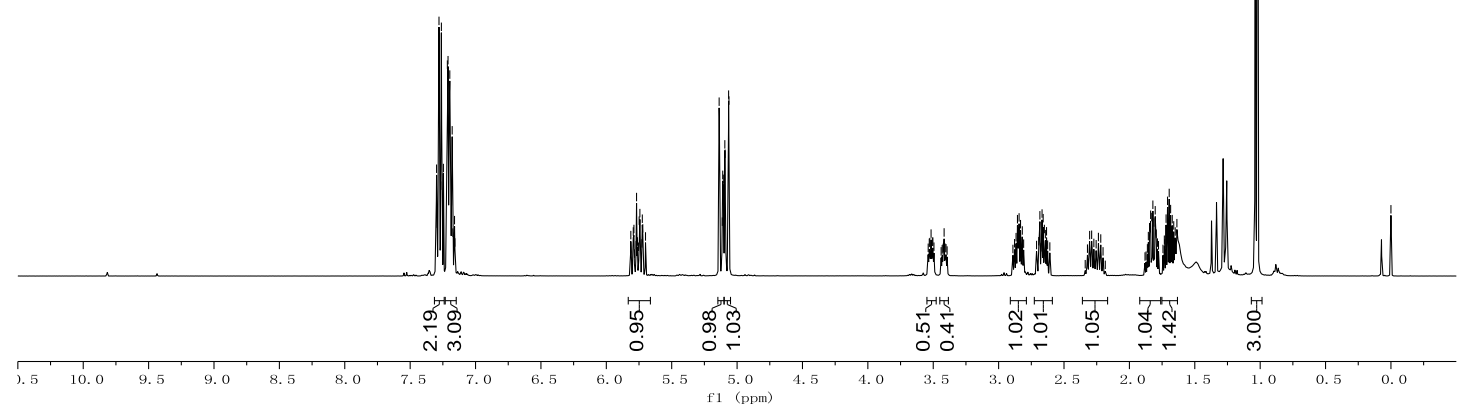

พิ่

光

m.

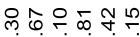

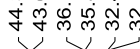

$1 \int_{23}^{\mathrm{OH}}$

定它走去

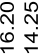

23

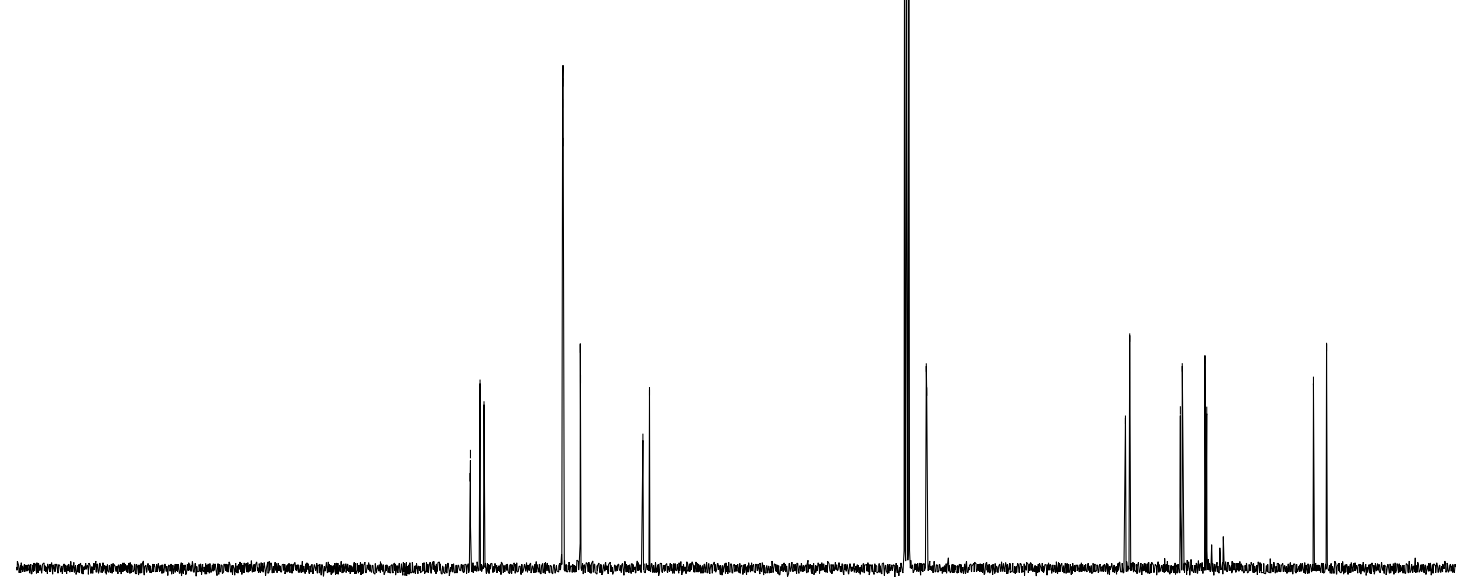

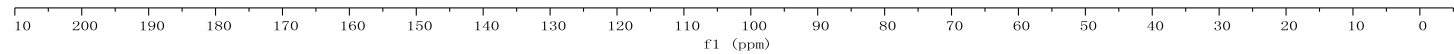



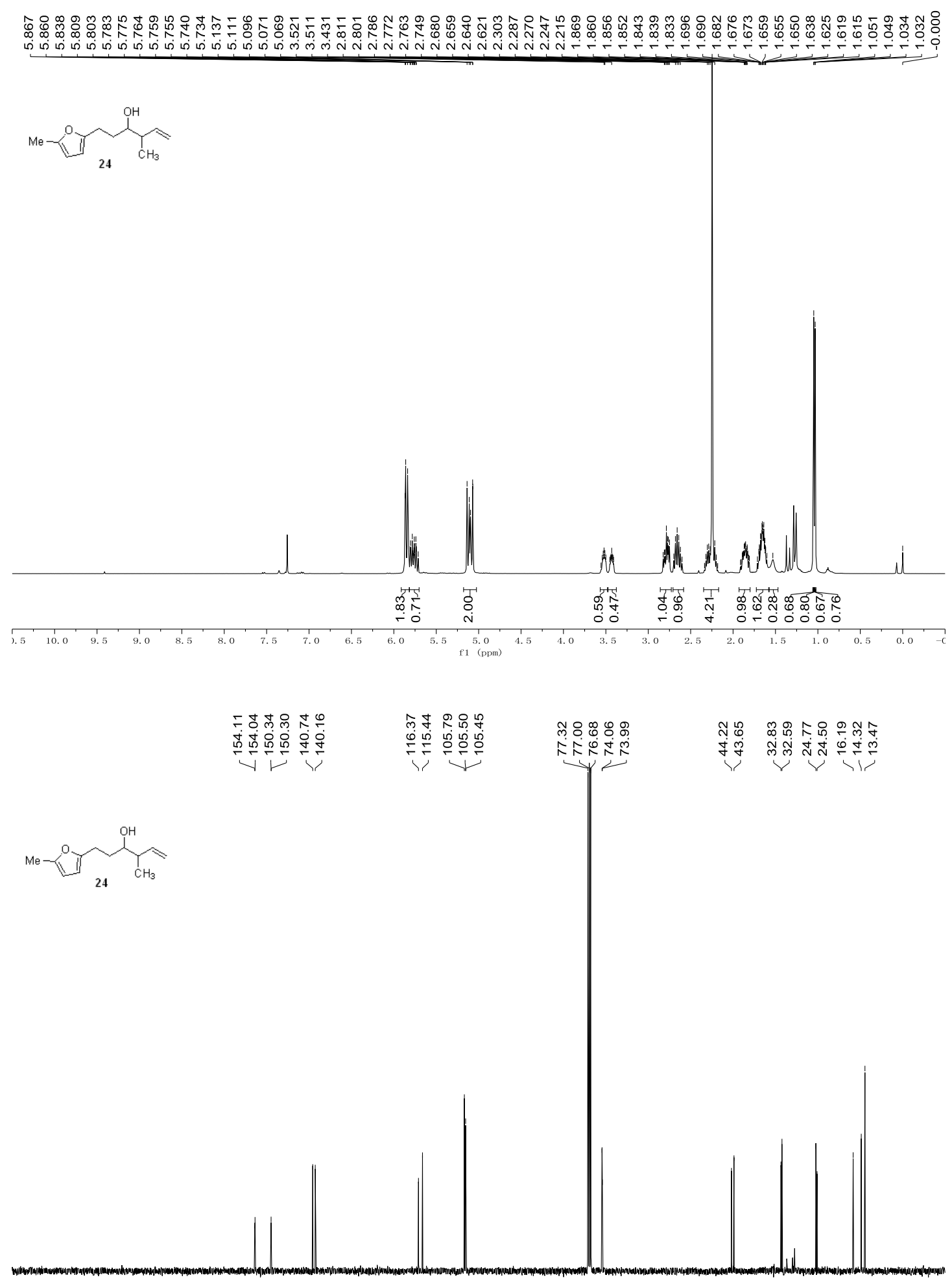

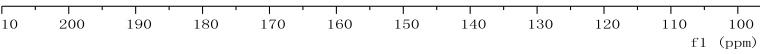




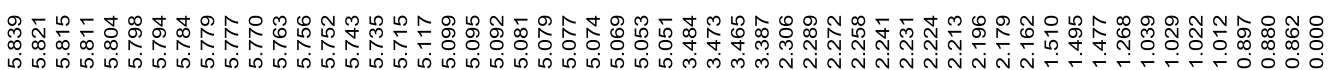

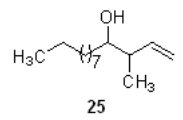

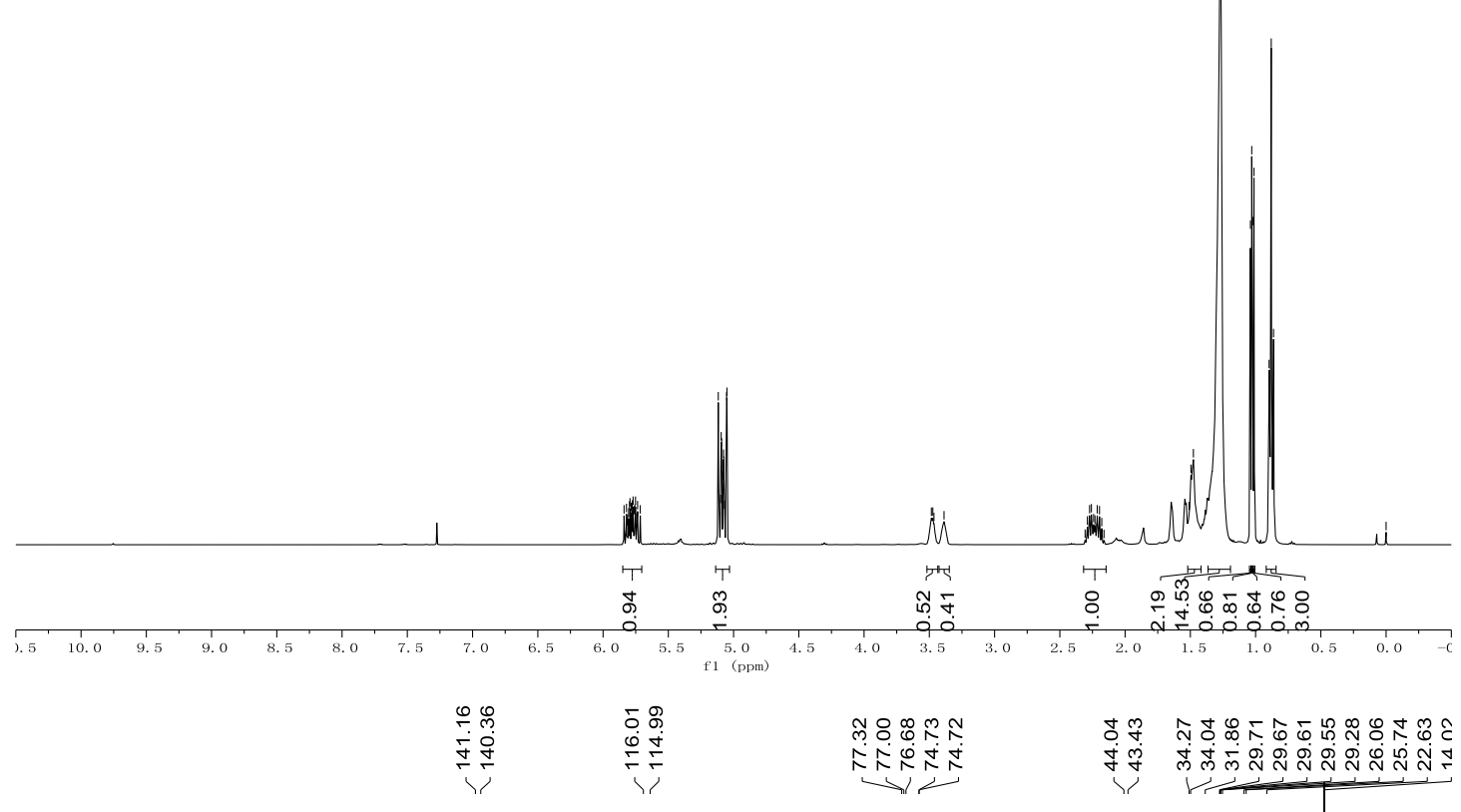

$\mathrm{H}_{3} \mathrm{C}>\mathrm{H}_{25}^{\mathrm{OH}} \mathrm{CH}_{3}^{\mathrm{O}}$

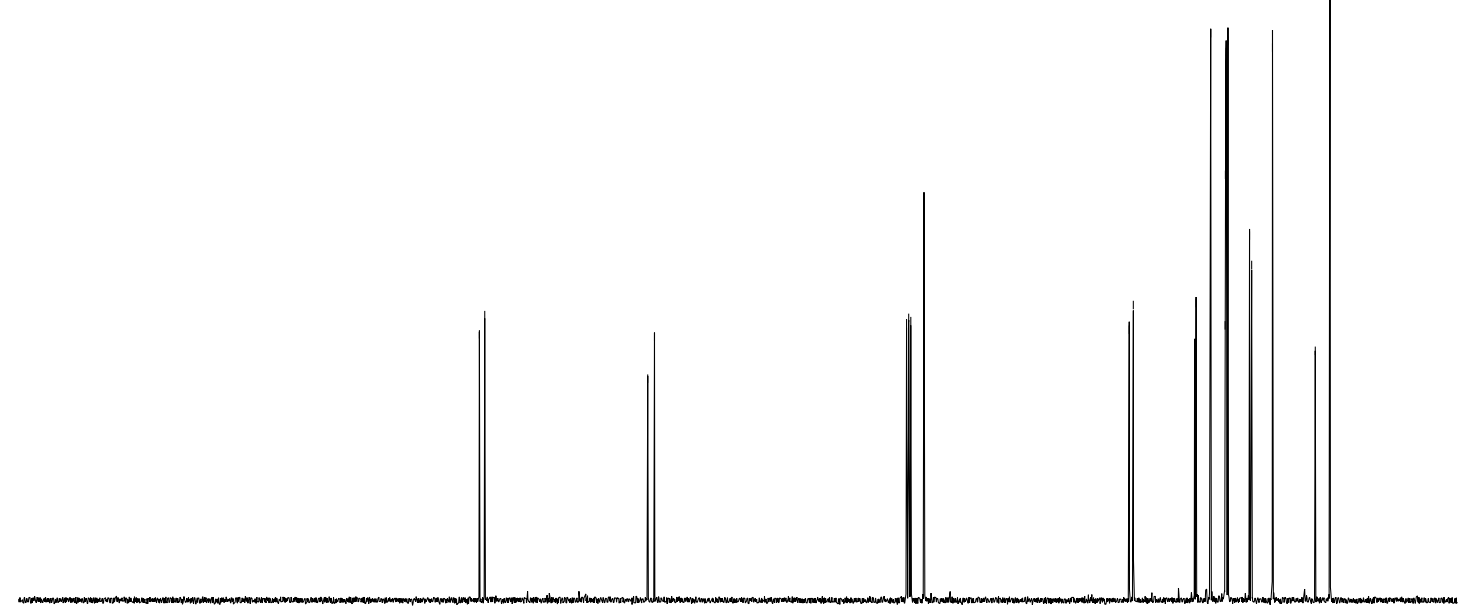

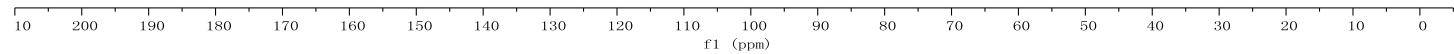




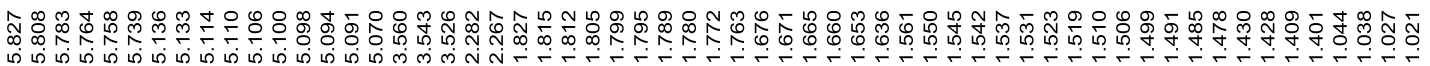

$\underbrace{\mathrm{OH}}_{\mathrm{CH}_{3}}$
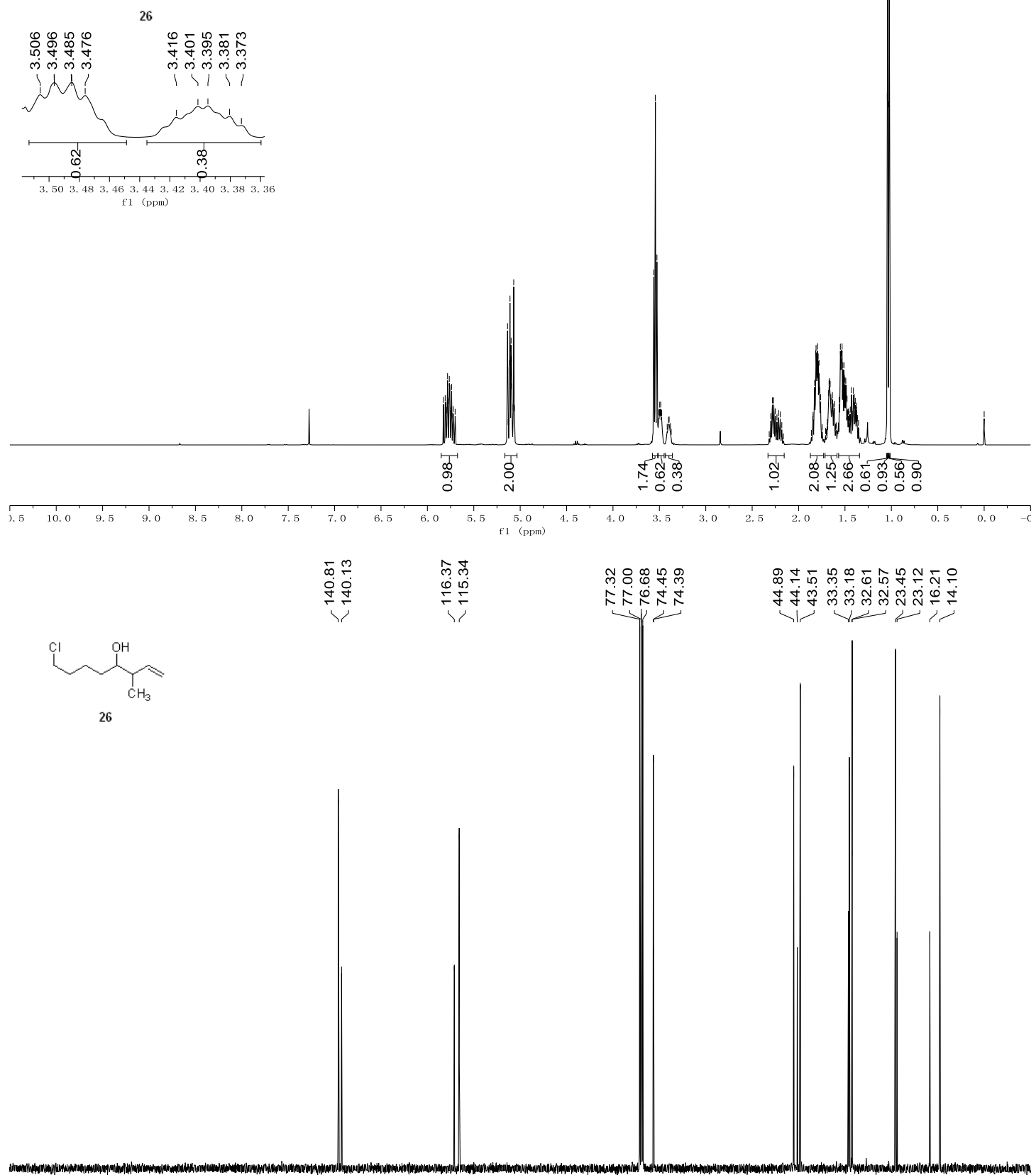

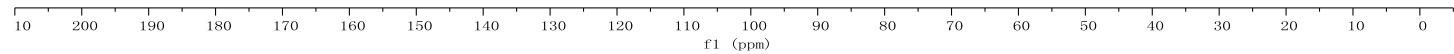




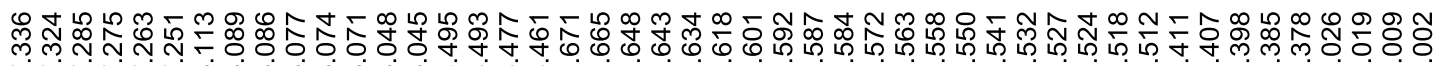

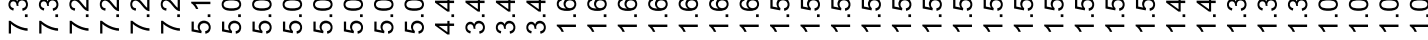
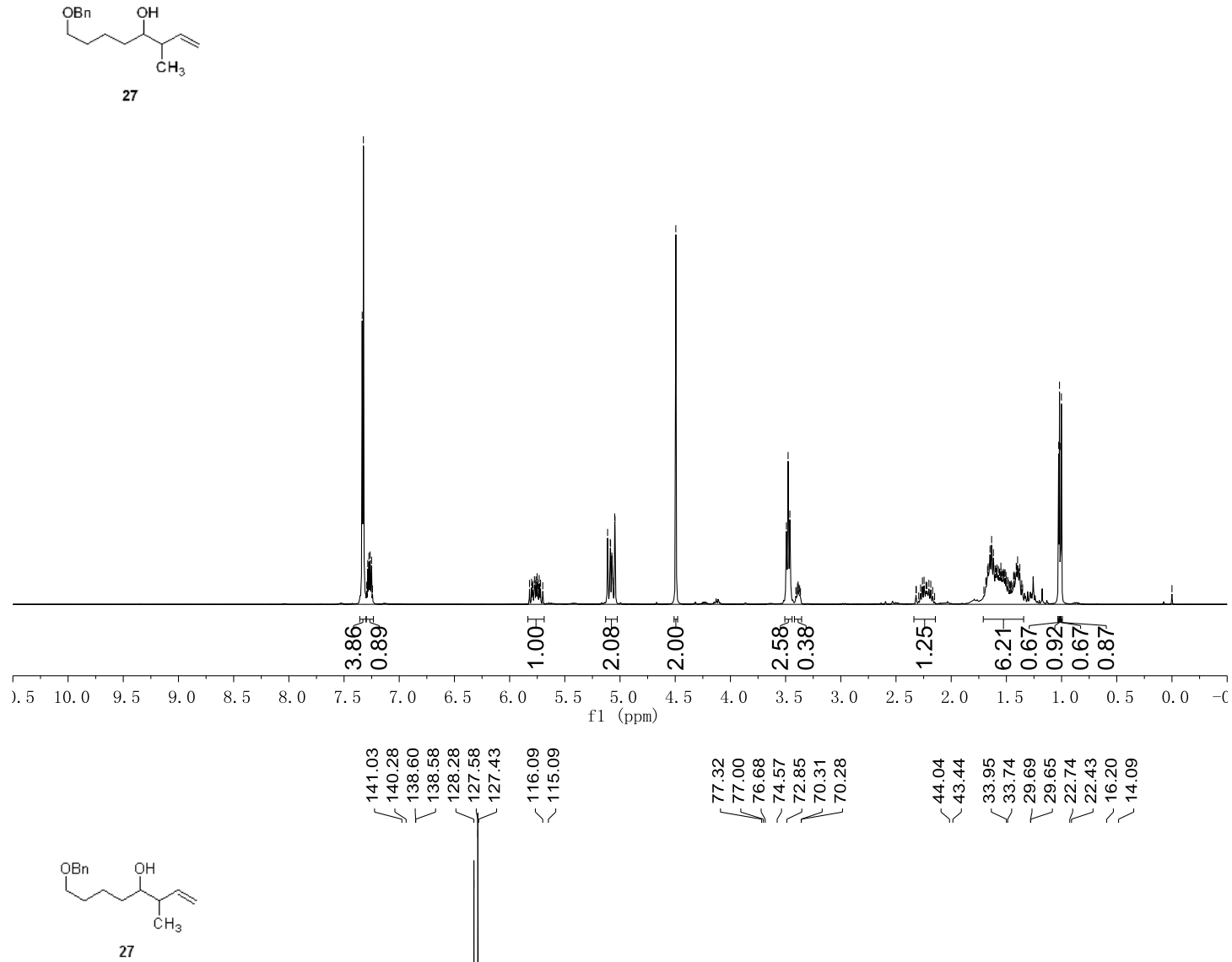

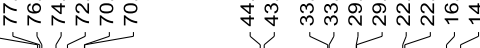

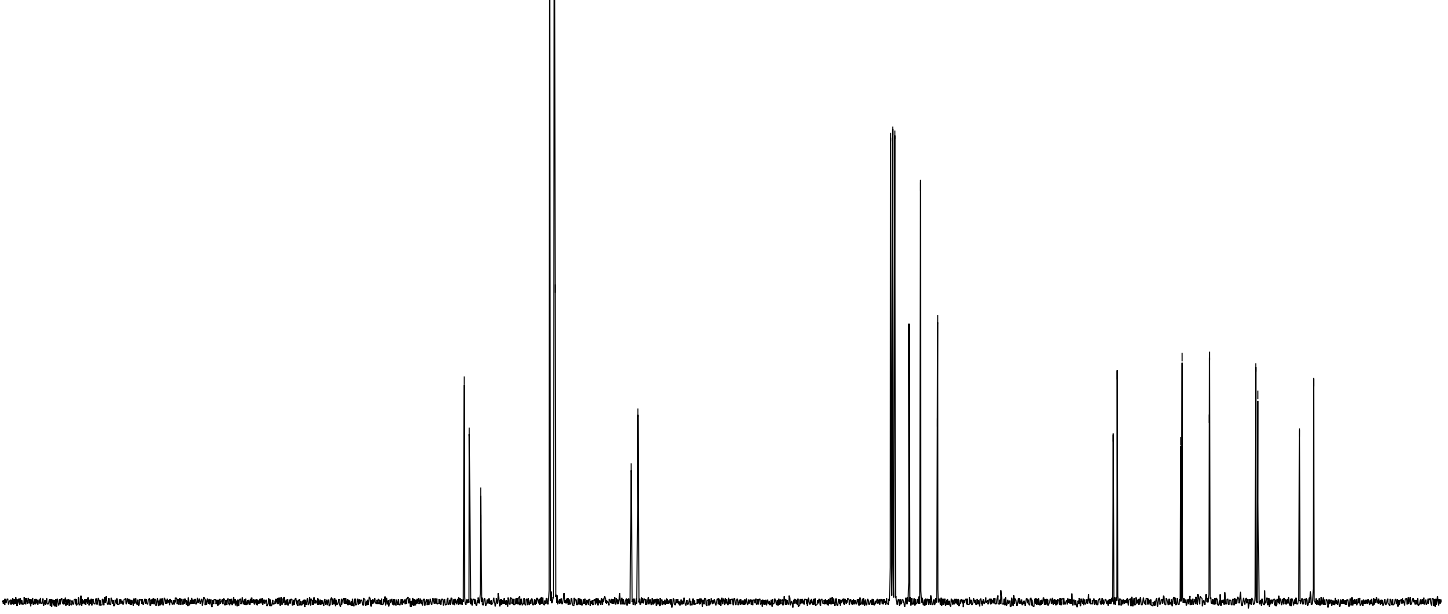

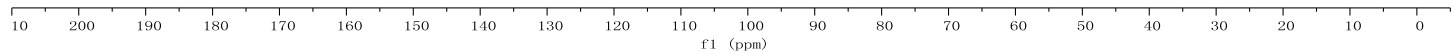




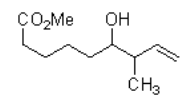

28

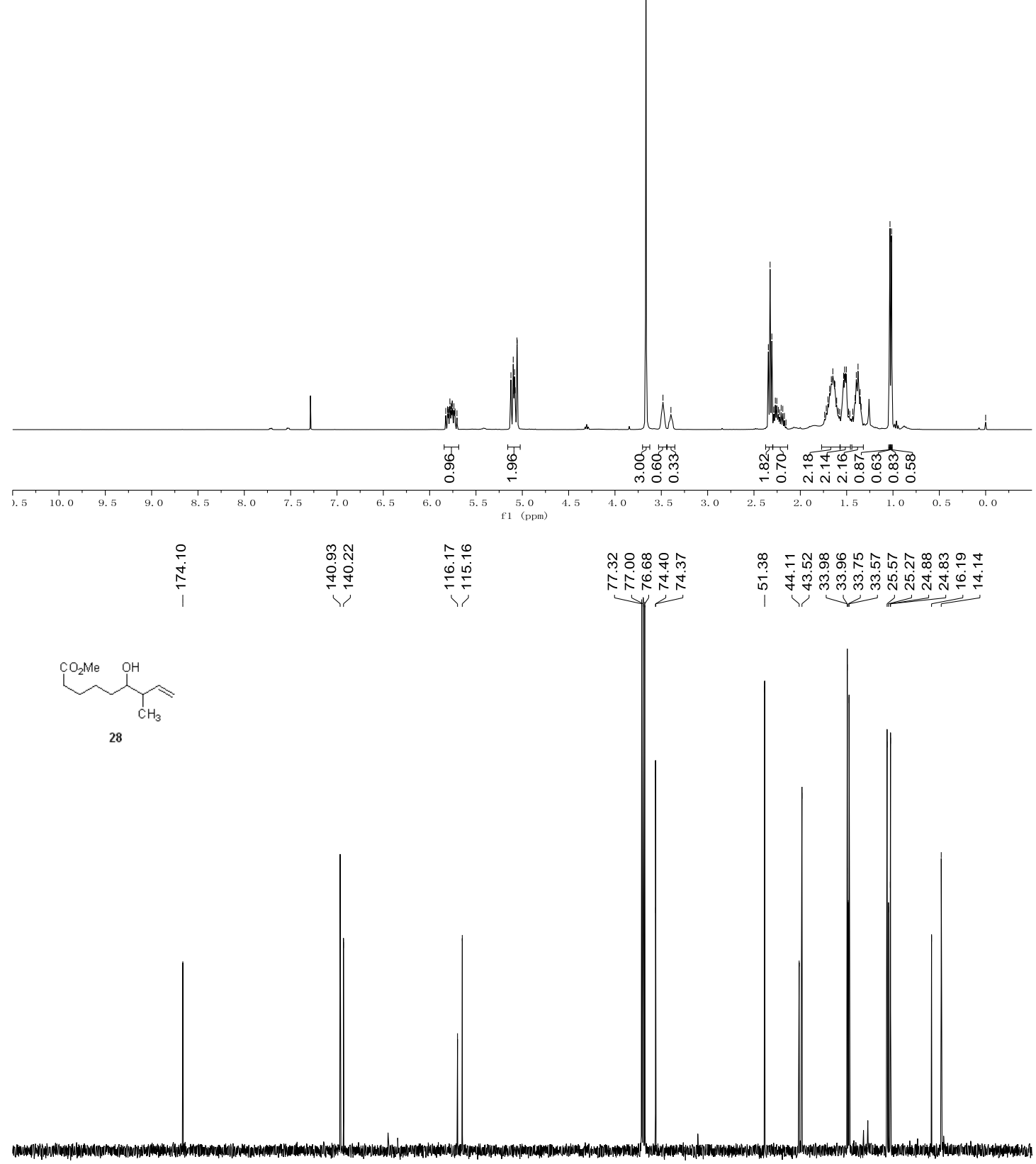

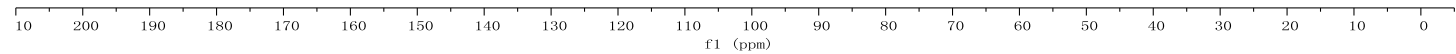




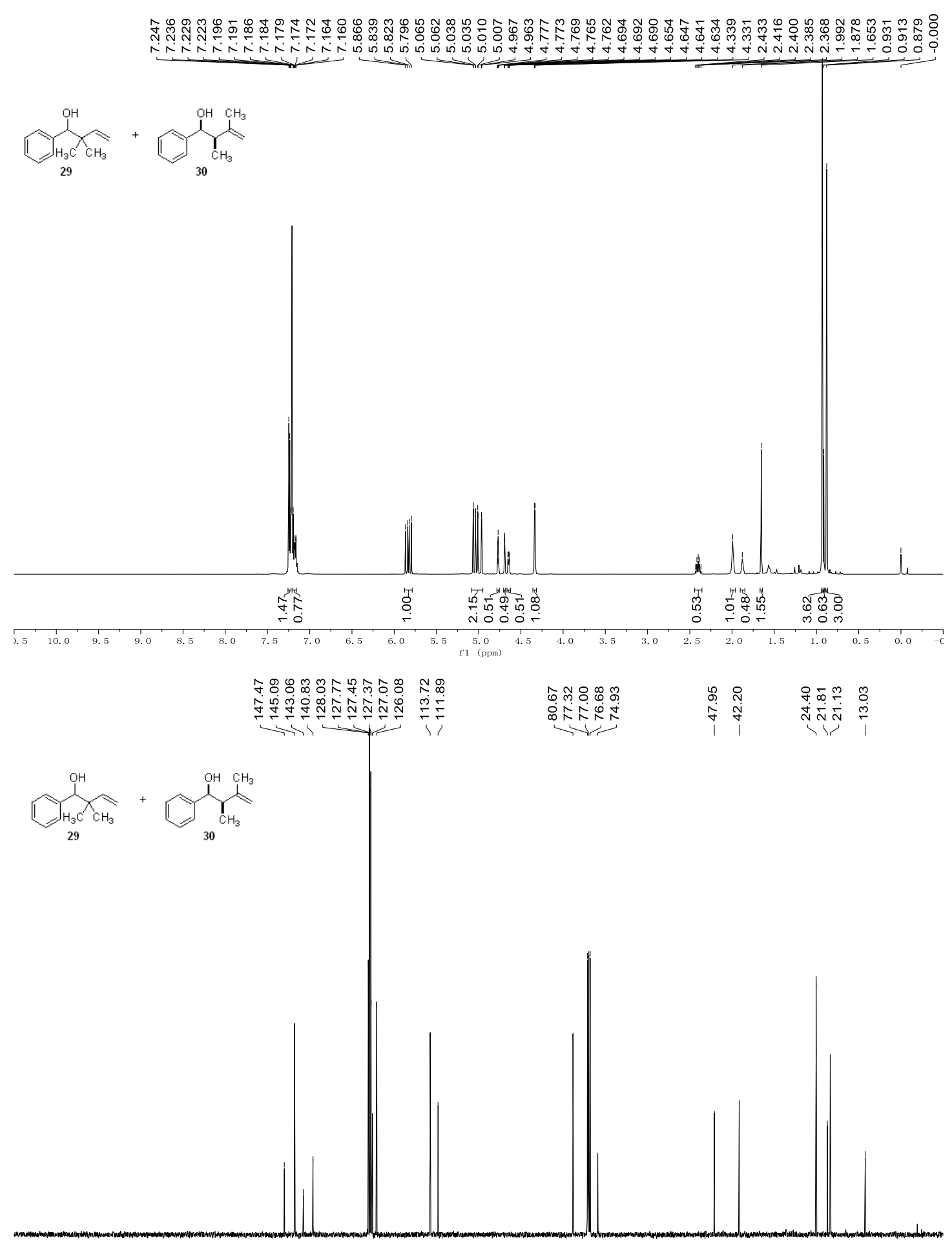

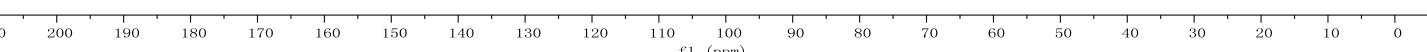



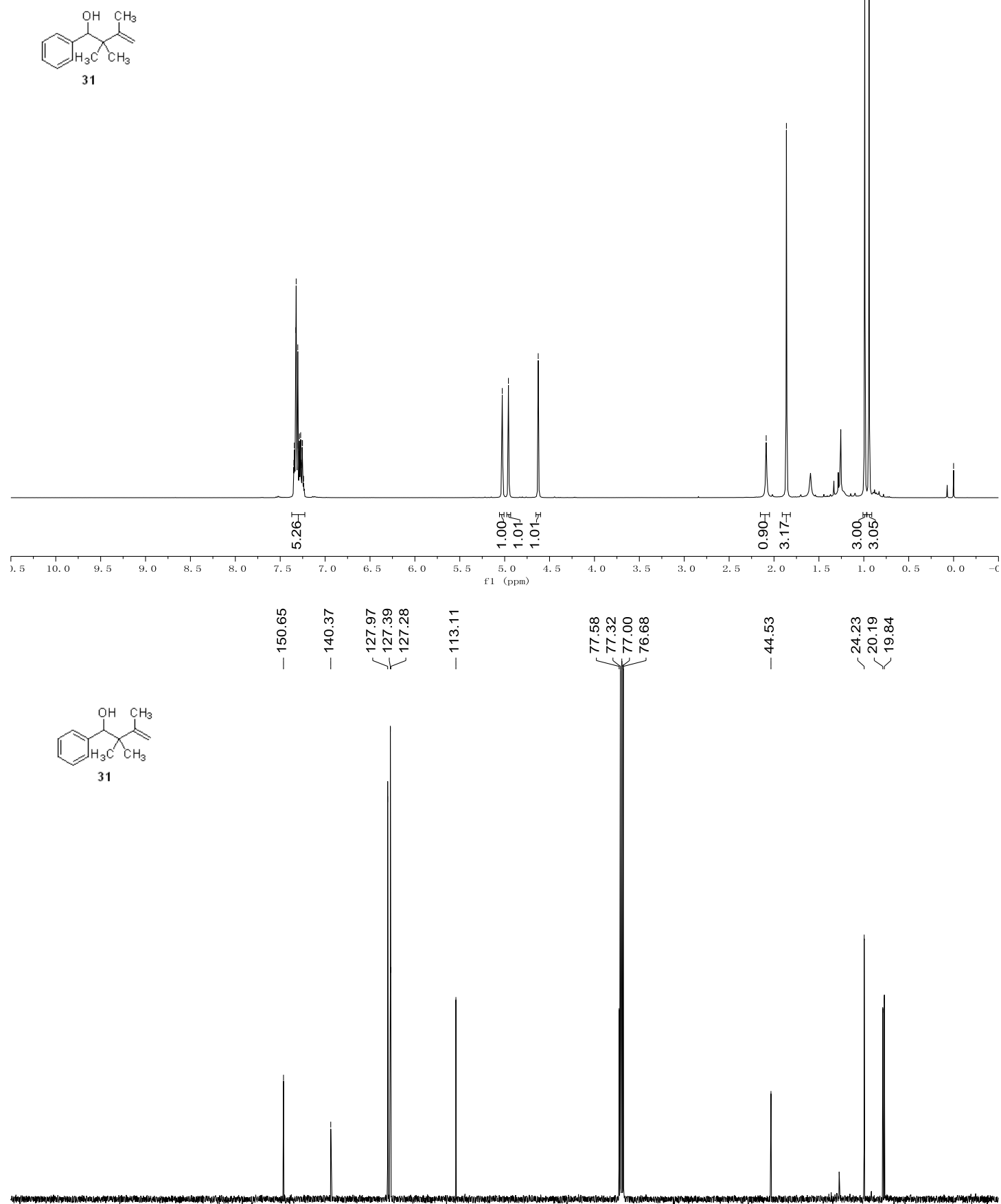

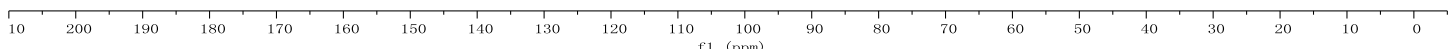




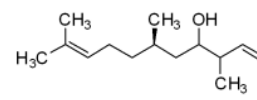

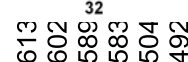

लं लं ले

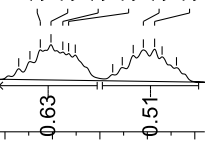

$3.60 \quad 3.50$

f1 (ppm)

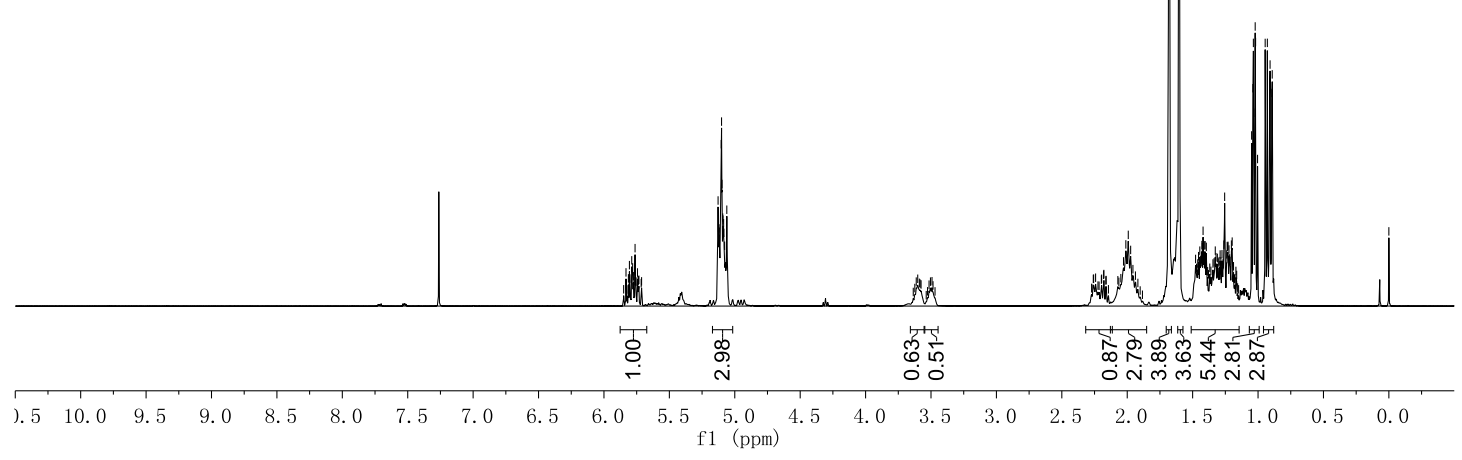

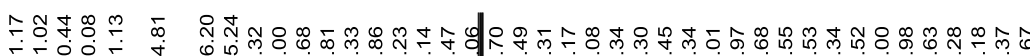

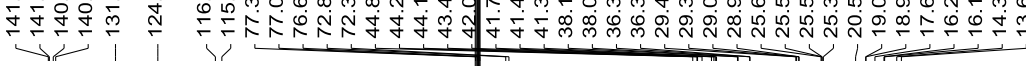

$\underbrace{\mathrm{H}_{3} \mathrm{C}}_{\mathrm{CH}_{3}}$

(1)

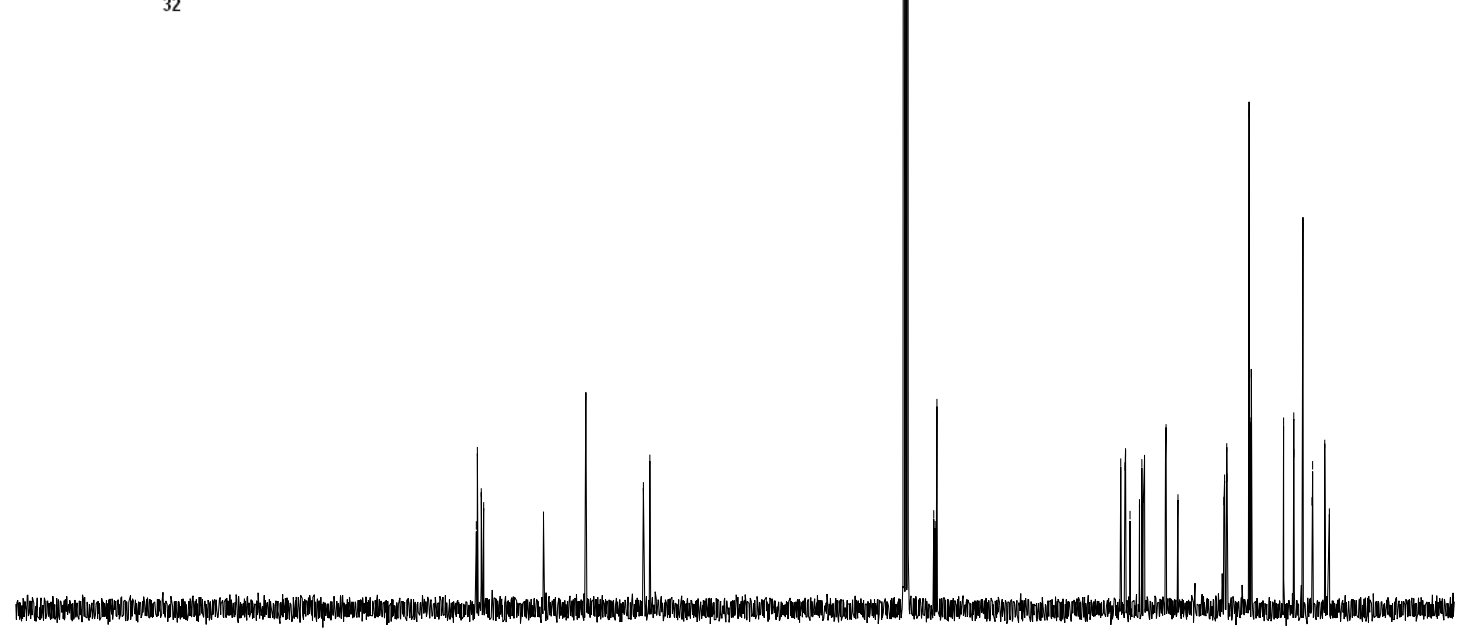

\begin{tabular}{llllllllllllllllllllll}
10 & 200 & 190 & 180 & 170 & 160 & 150 & 140 & 130 & 120 & 110 & 100 & 90 & 80 & 70 & 60 & $\frac{1}{10}$ & 40 & 30 & 20 & 10 & 1 \\
\hline
\end{tabular} 


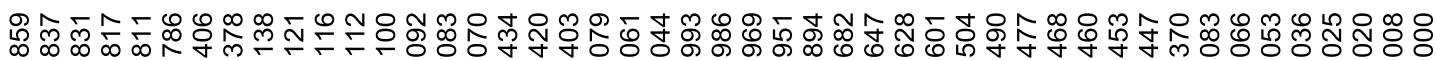

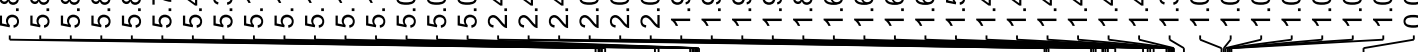

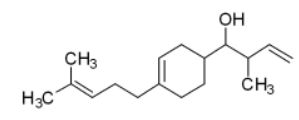

33

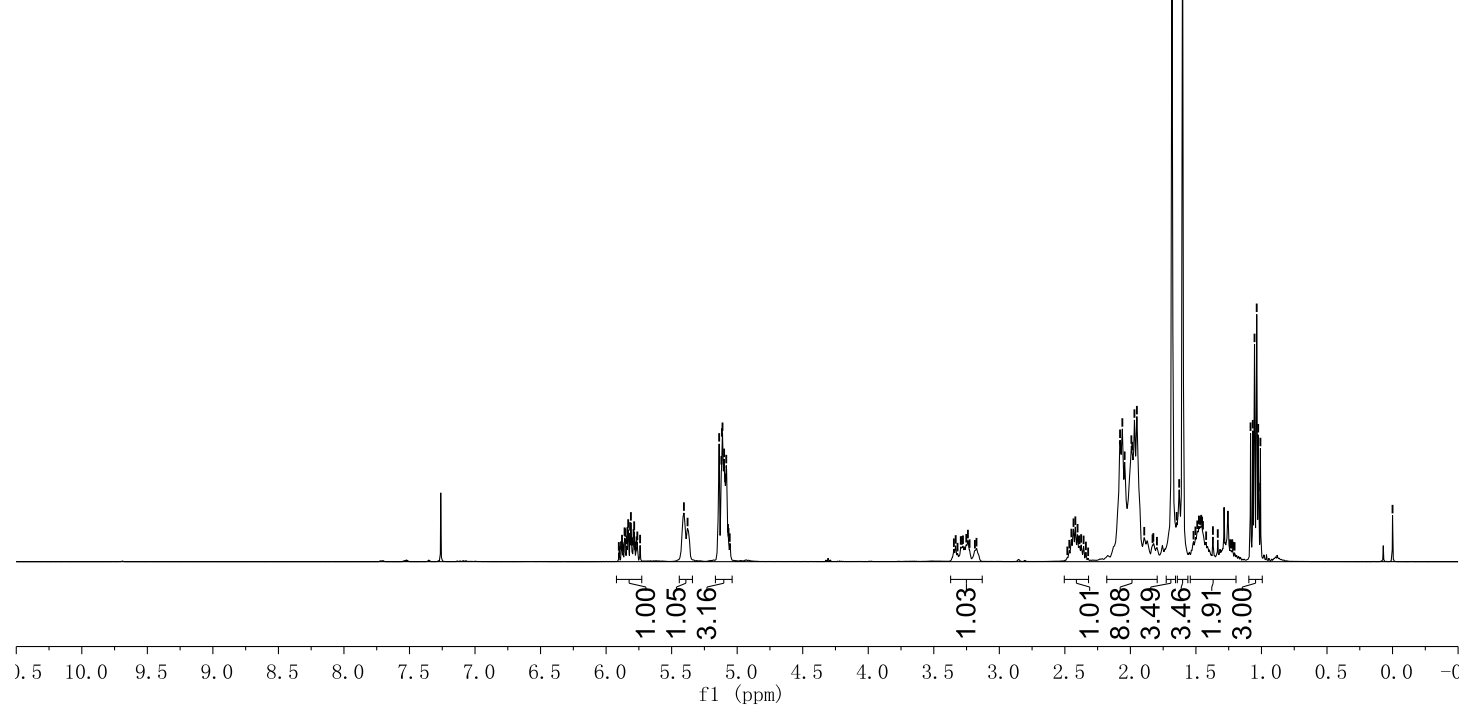

Г下

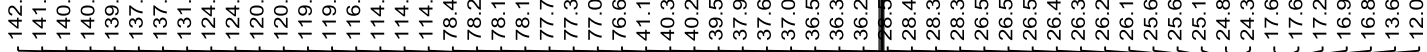

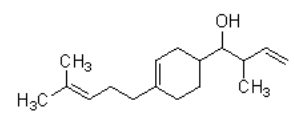

33

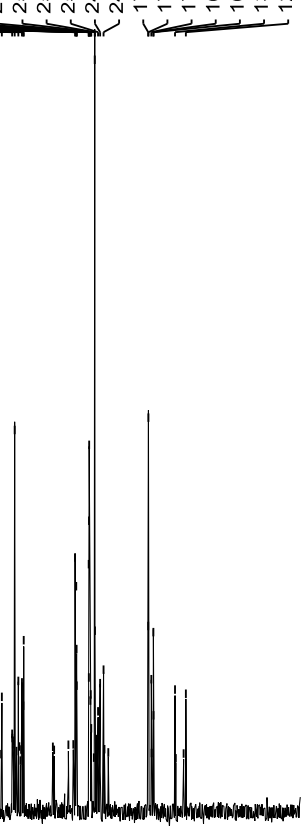

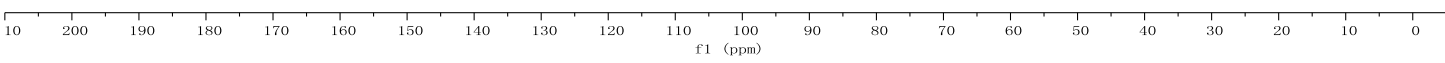




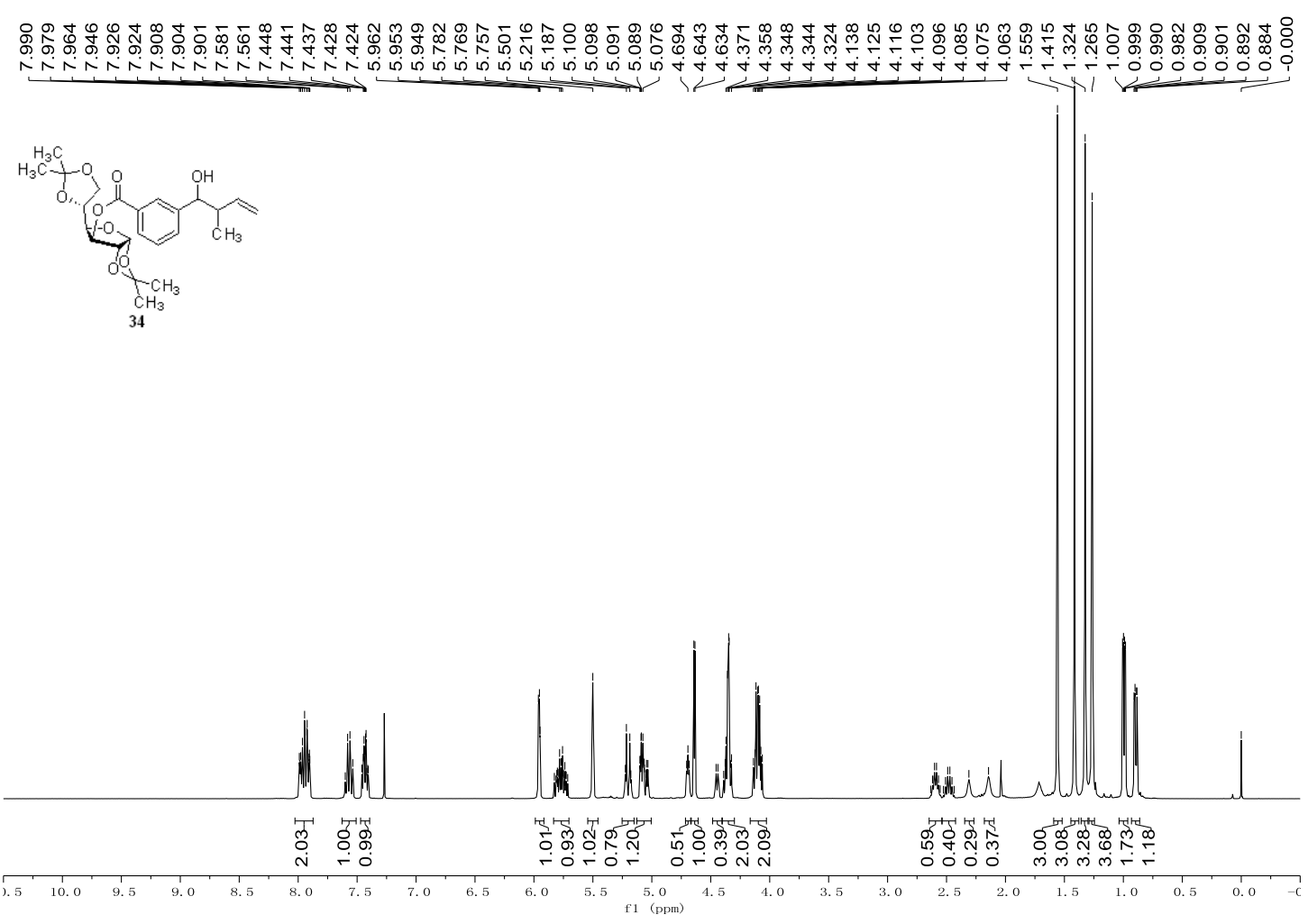

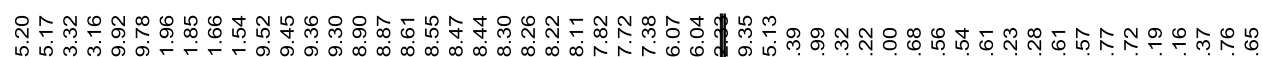
$\underbrace{.0000}$

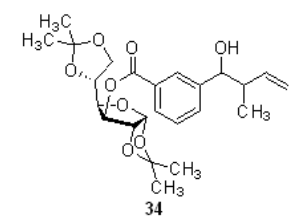

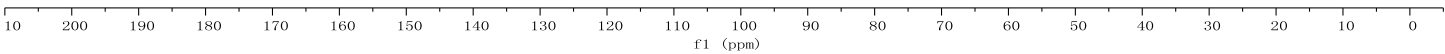




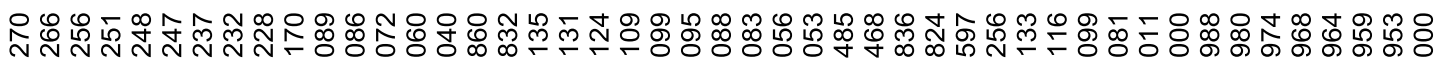
N<smiles>C=CC(C)C(O)C1CC1c1ccccc1</smiles>
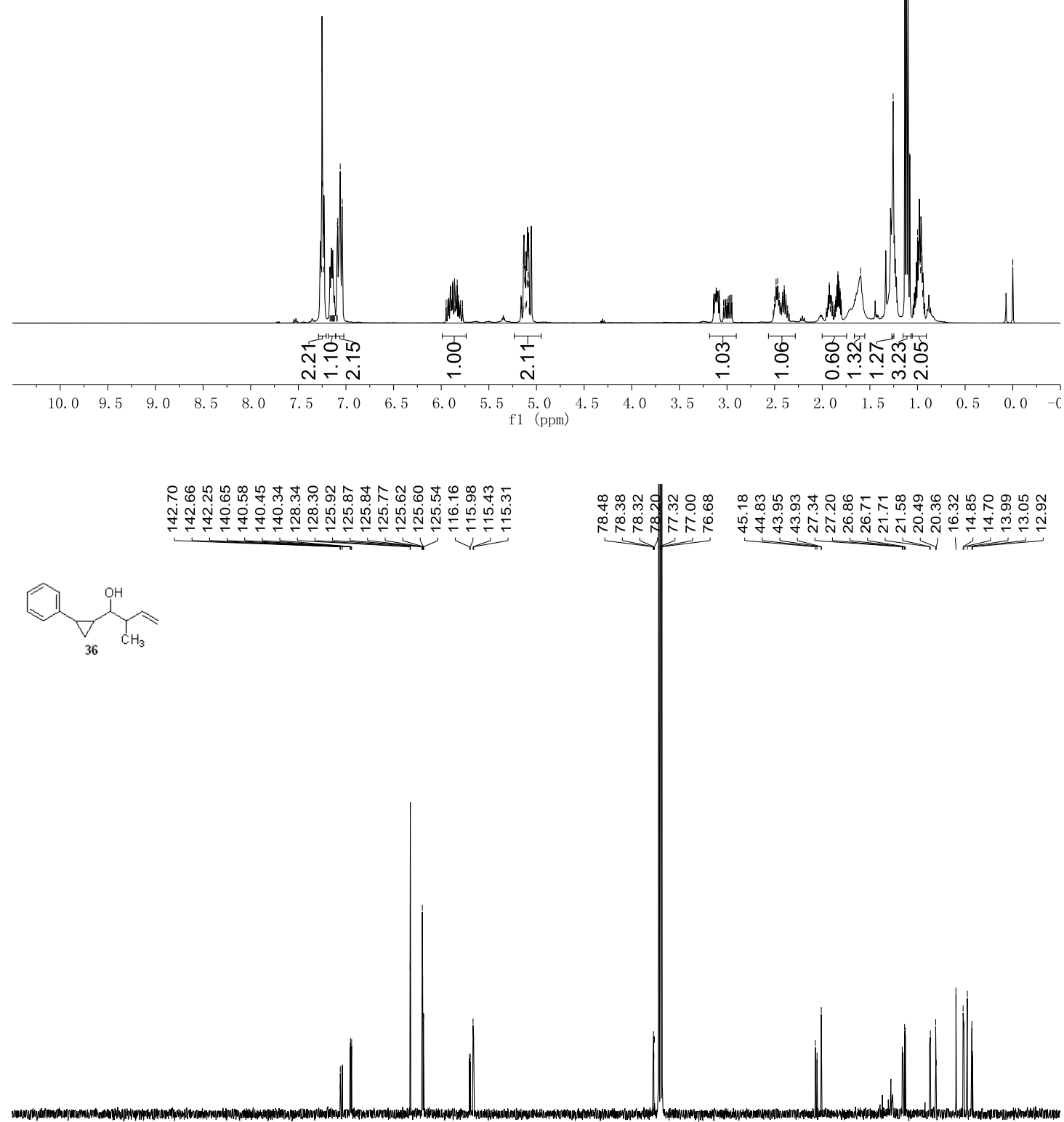

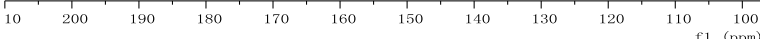

